Draft Version November 9, 2017

Preprint typeset using $\mathrm{IATE}_{\mathrm{E}} \mathrm{X}$ style emulateapj v. 12/16/11

\title{
AN EVALUATION OF COSMOLOGICAL MODELS FROM EXPANSION AND GROWTH OF STRUCTURE MEASUREMENTS
}

\author{
Zhongxu Zhai ${ }^{1}$, Michael Blanton ${ }^{1}$, Anže Slosar ${ }^{2}$, Jeremy Tinker ${ }^{1}$ \\ Draft version November 9, 2017
}

\begin{abstract}
We compare a large suite of theoretical cosmological models to observational data from the cosmic microwave background, baryon acoustic oscillation measurements of expansion, Type Ia SNe measurements of expansion, redshift space distortion measurements of the growth of structure, and the local Hubble constant. Our theoretical models include parametrizations of dark energy as well as physical models of dark energy and modified gravity. We determine the constraints on the model parameters, incorporating the redshift space distortion data directly in the analysis. To determine whether models can be ruled out, we evaluate the $p$-value (the probability under the model of obtaining data as bad or worse than the observed data). In our comparison, we find the well known tension of $\mathrm{H}_{0}$ with the other data; no model resolves this tension successfully. Among the models we consider, the large scale growth of structure data does not affect the modified gravity models as a category particularly differently than dark energy models; it matters for some modified gravity models but not others, and the same is true for dark energy models. We compute predicted observables for each model under current observational constraints, and identify models for which future observational constraints will be particularly informative.
\end{abstract}

Keywords: large scale structure of universe, dark energy

\section{INTRODUCTION}

Since it was first definitively discovered (Riess et al. 1998, Perlmutter et al. 1999), the accelerated expansion of the universe has been one of the greatest mysteries in modern physics. The attempt to explain this phenomena has motivated physicists to propose numerous theoretical models. Among these candidates, the cosmological constant is the mathematically simplest solution. Although the cosmological constant is able to fit the observations well, it has a critical problem: that its value indicated from cosmology differs from that estimated by quantum field theory by about $\sim 120$ orders of magnitude (Weinberg 1989).

Alternative approaches to explain cosmic acceleration without the cosmological constant include a number that introduce an exotic energy component in the universe, the so-called dark energy. Proposed models include dynamical vacuum energy, cosmic fluids, scalar fields and others, as we explain in detail below. Other approaches alter the geometrical structure of spacetime, i.e. they modify gravity. These models, some of them motivated by string theory or generalizations of General Relativity, introduce modifications of late time evolution of the universe, such as $f(R)$ gravity, $f(T)$ gravity, extra dimensions, Galileon cosmology, and a number of others described below. In this work, we focus on the models which are numerically economic and do not explicitly assume an exotic matter energy component. Recent reviews outline the theoretical motivation for this zoo of cosmological models (Peebles \& Ratra 2003, Copeland et al. 2006; Linder 2008; Silvestri \& Trodden 2009; Cald-

Email: zz681@nvu.edu

${ }^{1}$ Center for Cosmology and Particle Physics, Department of Physics, New York University, 4 Washington Place, New York, NY 10003, USA.

${ }^{2}$ Brookhaven National Laboratory, Upton, NY 11973, USA well \& Kamionkowski 2009, Weinberg et al. 2013, Joyce et al. 2015). Confronting this large family of theoretical scenarios, it is important to compare their predictions with observations of the expansion rate, large scale structure, and the cosmic microwave background, to determine whether the theories are viable and to constrain their parameters. Such comparisons are standard practice for both theorists and experimentalists, and wellestablished machinery exists to perform them.

Our work builds on these efforts in two major ways. First, the disparate investigations regarding the current models, conducted over a number of years and by many different groups, inevitably have used different data sets with different assumptions. In this work, we apply a consistent set of constraints to a large set of theories, to better understand them when analyzed within the same framework of observational data.

Second, while it is standard to constrain cosmological parameters with data, surprisingly not all investigations measure a "goodness-of-fit" of the models they study that is, how statistically incompatible the data are with each model. Here we do so for all models in a consistent fashion. We use the $p$-value as a measure of goodness-offit (Wasserstein \& Lazar 2016; Davis et al. 2007). The $p$ value is the probability within the model that one would observe data that, under some summary metric, is more different from the model than the actual data is. In evaluating the $p$-value we will account for the fact that we are free to set the model parameters. The particular metric we will use for the $p$-value is a global $\chi^{2}$ statistic. This approach requires care in interpretation, as we describe below, but does allow us to declare models whose $p$-values are intolerably low to be incompatible with the data.

We will use a combination of data to evaluate the models. The first convincing evidence of the cosmic accel- 
eration was from the observation of supernovae, soon followed by the measurement of degree-scale cosmic microwave background (CMB) anisotropies (Hinshaw et al. 2013 Planck Collaboration et al. 2014, 2015a), and later by data on the baryon acoustic oscillation (BAO) at lower redshifts (Sakharov 1966, Peebles \& Yu 1970; Sunyaev \& Zeldovich 1970; Blake \& Glazebrook 2003; Seo \& Eisenstein 2003). We combine the latest versions of these measurements with that of the local Hubble constant $\mathrm{H}_{0}$ (Riess et al. 2016). The combination of these measurements yields tight constraints on the parameters of the standard $\Lambda$ CDM model, and some dark energy models. Under most such models, the local Hubble constant $\mathrm{H}_{0}$ measurements are in tension with the higher redshift constraints. The models that can resolve this tension are thus of importance to investigate, and this is one of the goals in our work.

We also take advantage of the recent measurements of the linear growth of perturbations, which complements the above geometrical probes. This observation provides information on the dynamics of the cosmology which might play an important role in dark energy model selection. Our work introduces a simple technique to incorporate these measurements of growth directly into cosmological constraints in a manner compatible with the analysis of most modified gravity and dark energy models. We will use this technique to explore in detail the interplay of these different datasets, and evaluate the performance of different models given the data.

Our paper is organized as follows. In Section 2, we introduce the dark energy and modified gravity models briefly, as well as the datasets we use. Section 3 details our approach to evaluate the models by their $p$-values. Section 4 presents the cosmological constraints for each model with the predictions of the geometrical and dynamical observable from current data, and compares with future experiments. We discuss and list our conclusions in Section 5.

\section{COSMOLOGICAL MODELS AND DATA SETS}

A homogeneous and isotropic universe is described by the Friedman-Robertson-Walker metric

$$
d s^{2}=-d t^{2}+a^{2}(t)\left[\frac{d r^{2}}{1-k r^{2}}+r^{2} d \Omega^{2}\right],
$$

where $a(t)$ is the scale factor related to redshift $z$ as $a(t)=(1+z)^{-1}$ and $k$ is the curvature parameter. In General Relativity (GR), the evolution of the universe is governed by the field equation which connects the Einstein tensor and energy momentum tensor

$$
G_{\mu \nu}=8 \pi G T_{\mu \nu},
$$

where $G$ is the Newton gravitational constant. This equation gives the expansion of the universe as

$$
\frac{H^{2}}{H_{0}^{2}}=E^{2}(z)=\frac{\rho(a)}{\rho_{0}}+\Omega_{k} a^{-2},
$$

where $H \equiv \dot{a} / a$ is the Hubble parameter, $E(z)$ is a dimensionless function which is sometimes called expansion factor, $\rho(a)$ is the total energy density, the subscript ' 0 ' denotes the value at present $(z=0)$. The density parameter of a given energy density is defined as

$$
\Omega_{x}=\frac{\rho_{x}}{\rho_{\text {crit }}}=\frac{8 \pi G}{3 H^{2}} \rho_{x} .
$$

The normalization of the Hubble parameter gives the relation between the density parameter and curvature parameter

$$
\sum_{x} \Omega_{x}+\Omega_{k}=1
$$

where the summation is over all the energy and matter components in the universe. Throughout this paper, the density parameters refer to the current values at $z=0$ unless otherwise denoted.

In the standard $\Lambda \mathrm{CDM}$ model, the dark energy is a constant in space and time, therefore we can write the Hubble parameter as

$\frac{H^{2}}{H_{0}^{2}}=E^{2}(z)=\Omega_{m}(1+z)^{3}+\Omega_{r}(1+z)^{4}+\Omega_{\Lambda}+\Omega_{k}(1+z)^{2}$,

where $\Omega_{m}$ includes the contribution from cold dark matter $(\mathrm{CDM})$ and baryons, $\Omega_{r}$ represents radiation and relativistic matter, and $\Omega_{\Lambda}$ is the cosmological constant. For simplicity and the efficiency of the calculation, we assume the neutrinos are massless and contribute the same as radiation.

Below, we briefly introduce the dark energy and modified gravity models that are explored in this paper. Table 2.1.2.5 summarizes the models we consider and their compatibility with the linear growth data and local $\mathrm{H}_{0}$ measurement.

\subsection{Cosmological models}

We divide the alternative models under consideration into two categories: dark energy models, motivated by new components or physical effects, and modified gravity models, which alter the nature of general relativity.

\subsubsection{Dark energy models}

2.1.1.1. Parameterizations of the equation of state $w$ (XCDM, $\left.C P L, J B P w_{\text {Linear }}\right)$ - The equation of state $w$ of a perfect fluid is defined as the ratio of its pressure and density

$$
w=p / \rho \text {. }
$$

For cosmological constant, we have $w=-1$. Therefore the simplest generalization is $w=$ constant (Turner \& White 1997, Chiba et al. 1997) which changes the third term on the right hand side of Eq. (6) to be

$$
\Omega_{\Lambda}(1+z)^{3(1+w)} \text {. }
$$

In this kind of cosmology (hereafter XCDM), the DE models with a conical kinetic term have $-1<w<-1 / 3$, while models with $w<-1$ are phantom dark energy (Faraoni \& Dolgov 2002, Caldwell 2002, Caldwell et al. 2003). Thus $w=-1$ is a critical value to differentiate the phantom property of the dark energy.

The generalization of constant $w$ is to assume it is a function of time. The first model considered in this paper is the CPL parameterization (Chevallier \& Polarski 2001, Linder 2003), which is the first order Taylor expansion of the scale factor at the present epoch

$$
w=w_{0}+w_{1}(1-a)=w_{0}+w_{1} \frac{z}{1+z},
$$


where $w_{0}$ and $w_{1}$ are two parameters. The corresponding term for dark energy Eq. (8) needs to be

$$
\Omega_{\Lambda} \exp \left(3 \int_{0}^{z} \frac{1+w}{1+z^{\prime}} d z^{\prime}\right) .
$$

Another type of parameterization is proposed in Jassal et al. (2005) (hereafter JBP):

$$
w=w_{0}+w_{1} \frac{z}{(1+z)^{2}} .
$$

Note that this model has quite different asymptotic behavior at high redshift than does the CPL parameterization.

The last model we consider here is a simple linear function of redshift $z$ (hereafter $w_{\text {Linear }}$, Cooray \& Huterer 1999, Weller \& Albrecht 2002; Astier 2001)

$$
w=w_{0}+w_{1} z,
$$

These three parameterizations have similar behavior in the late time universe. Note that the integral in Eq. 10) can be calculated analytically, therefore no numerical routine is needed, which is important in the following computations.

2.1.1.2. Pseudo-Nambu Goldstone Boson (PNGB) - This model is motivated by the potential $V(\phi \propto[1+$ $\cos (\phi / F)]$ ) of the scalar field (Frieman et al. 1995; Dutta \& Sorbo 2007). The dark energy in this model is parameterized by its equation of state (Basilakos et al. 2010)

$$
w=-1+\left(1+w_{0}\right)(1+z)^{-F},
$$

where $w_{0}$ and $F$ are free parameters. When $w_{0}=-1$, this model becomes standard $\Lambda$ CDM model. Because $z>0$, the second term also vanishes as $F$ becomes large, so we restrict the range of $F$ to be within $[0,8]$ as adopted in literature.

2.1.1.3. Casimir effect (CE) - This kind of cosmology involves a negative radiation-like term in the expression of Hubble parameter Eq. (6):

$$
\Omega_{c}(1+z)^{4},
$$

where $\Omega_{c}<0$ is a constant Godłowski \& Szydłowski 2006). In principle, there are different interpretations of this term. The first is the Casimir effect due to the vacuum energy and its relationship to the curvature and topology of the universe (Bordag et al. 2001). The second is called "dark radiation" and is introduced by the extra dimension in the universe. For instance, in the Randall \& Sundrum (1999) scenario, the restriction of the field equation on the brane induces this term. The third interpretation that it arises from the global rotation of the universe (Senovilla et al. 1998, Godłowski \& Szydłowski 2006).

2.1.1.4. Cardassian Ansatz (CA) - The Cardassian expansion model was first proposed by Freese \& Lewis (2002). It is a modification of the Friedmann equation from $H^{2}=A \rho$ to

$$
H^{2}=A \rho+B \rho^{n},
$$

with the parameter $n<2 / 3$. Assuming the curvature and radiation are ignored, this equation can be written in terms of the density parameters as

$$
E^{2}(z)=\Omega_{m}(1+z)^{3}+\left(1-\Omega_{m}\right)(1+z)^{-3 n} .
$$

Therefore, it is equivalent to the XCDM model with $w=$ $n-1$. This model is generalized in Wang et al. (2003) by introducing one more free parameter $q>0$. The resulting Hubble parameter is expressed as:

$$
E^{2}(z)=\Omega_{m}(1+z)^{3}\left(1+\frac{\Omega_{m}^{-q}-1}{(1+z)^{3 q(1-n)}}\right)^{\frac{1}{q}} .
$$

This model is also called "Modified Polytropic Cardassian" when it is treated as a fluid. However, this model can also arise from the self-interaction of dark matter, or embedding the observable brane into a higher dimensional universe (Davis et al. 2007). This is the model (CA hereafter) tested in this paper.

2.1.1.5. Early dark energy (EDE) - In many models, dark energy is important only in the late time universe. However, in some models the dark energy tracks the evolution of the dominant component in the universe. In those cases, dark energy can produce an imprint during the early time of the universe. We consider a generic parameterization of such early dark energy models as proposed by Doran \& Robbers (2006). In this model, the density parameter of dark energy is given as a function of $a=(1+z)^{-1}$

$$
\Omega_{\mathrm{de}}=\frac{\Omega_{\mathrm{de}}^{0}-\Omega_{\mathrm{de}}^{\mathrm{e}}\left(1-a^{-3 w_{0}}\right)}{\Omega_{\mathrm{de}}^{0}+\Omega_{m} a^{3 w_{0}}}+\Omega_{\mathrm{de}}^{\mathrm{e}}\left(1-a^{-3 w_{0}}\right),
$$

where $\Omega_{\mathrm{de}}^{\mathrm{e}}$ and $\Omega_{\mathrm{de}}^{0}$ are the dark energy density parameter at early times and at present respectively. Therefore this model is fully characterized by these two parameters and $w_{0}$, which is the effective value of the equation of state. The Hubble parameter is given by

$$
E^{2}(a)=\frac{\Omega_{m} a^{-3}+\Omega_{r} a^{-4}}{1-\Omega_{\mathrm{de}}} .
$$

Note that this model approaches $\Lambda$ CDM model when $w_{0}=-1$ and $\Omega_{\mathrm{de}}^{\mathrm{e}}$ goes to zero.

2.1.1.6. Slow roll dark energy (SR) - The late time acceleration of the universe is similar to inflation. Therefore, it is possible that these two phenomena arise from the same physical mechanism. Based on this idea, Gott \& Slepian (2011); Slepian et al. (2014) propose a simple dark energy model. This model class is a slowly rolling scalar field with potential $\frac{1}{2} m^{2} \phi^{2}$. The dark energy is parameterized by the equation of state:

$$
w(z)=-1+\delta w(z) \approx \delta w_{0} \times H_{0}^{2} / H^{2}(z),
$$

and therefore

$$
E^{2}(z)=\Omega_{m}(1+z)^{3}+\Omega_{\mathrm{de}}\left[\frac{(1+z)^{3}}{\Omega_{m}(1+z)+\Omega_{\mathrm{de}}}\right]^{\delta w_{0} / \Omega_{\mathrm{de}}},
$$

where $\delta w_{0}$ is the first order term in the deviation at $z=0$ and represents the free parameter in this class of model.

2.1.1.7. Parameterization of the Friedmann equation (Poly$C D M, H L G)$ - The energy density of dark energy can be described by a quadratic polynomial form with non-zero space curvature. This model is highly flexible regarding 
the evolution of dark energy at low redshift Aubourg et al. 2015). The Hubble parameter is given as

$$
\begin{aligned}
E^{2}(z)= & \Omega_{m}(1+z)^{3}+\Omega_{k}(1+z)^{2}+\Omega_{1}(1+z)^{2} \\
& +\Omega_{2}(1+z)^{1}+\left(1-\Omega_{m}-\Omega_{k}-\Omega_{1}-\Omega_{2}\right),
\end{aligned}
$$

where $\Omega_{1}$ and $\Omega_{2}$ are two extra parameters. This Polynomial CDM (PolyCDM) model can also be thought of as a parameterization of the Hubble parameter.

For comparison, we consider the Logarithmic Hubble model (hereafter HLG) inspired from $f(R)$ gravity (Capozziello et al. 2014). We defer the discussion of $f(R)$ gravity in general to Sec. 2.1.2.2, and treat the HLG model as a simple parameterization of the Hubble parameter

$$
E^{2}(z)=\Omega_{m}(1+z)^{3}+\Omega_{k}(1+z)^{2}+\log (\alpha+\beta z),
$$

where $\alpha$ and $\beta$ are parameters with $\alpha=\exp \left(1-\Omega_{m}-\Omega_{k}\right)$, so only $\beta$ is the free parameter.

2.1.1.8. Chaplygin gas (CG, GCG, $M C G)$ - The Chaplygin gas (CG) was first introduced in aerodynamics in 1904. It was applied to cosmology by Kamenshchik et al. (2001) as an unified fluid of dark matter and dark energy, which has an equation of state

$$
p=-\frac{A}{\rho},
$$

where $p$ and $\rho$ are pressure and energy density in a comoving reference frame, and $A$ is a positive constant. This model can also arise in string theory models (Bordemann \& Hoppe 1993).

This model can be extended to the Generalized Chaplygin gas (GCG) which has the equation of state (Bento et al. 2002, Bilić et al. 2002)

$$
p=-\frac{A}{\rho^{\alpha}},
$$

where $\alpha$ is a new parameter with $0<\alpha<1$. In this model, the pressure in high redshift is negligible, while at late times both the pressure and energy density are constant. Therefore this single fluid can describe the matter-dominated universe until late time acceleration. This model can also arise from a complex scalar field whose action can be written as a generalized Born-Infeld action corresponding to a "perturbed" $d$-brane in a $(d+$ 1,1) spacetime (Bento et al. 2002).

GCG model can also be extended by adding a term, yielding the Modified Chaplygin gas (MCG) model (Benaoum 2002, hereafter MCG), with the equation of state

$$
p=B \rho-\frac{A}{\rho^{\alpha}},
$$

where $B$ is the a new constant. As mentioned above, these classes of Chaplygin gas model were first proposed as a unified description of dark matter and dark energy. However, previous study of perturbation theory finds that growth of structure in this unified description is inconsistent with cosmological data on perturbations (Bean \& Doré 2004, Giannantonio \& Melchiorri 2006). Therefore, here we assume that the Chaplygin gas only contributes to the dark energy component. After redefinition of the parameter $A_{s}=\frac{A}{1+B} \frac{1}{\rho_{0}^{1+\alpha}}$, where $\rho_{0}$ is the current energy density of dark energy, the Hubble parameter for MCG model can be written as

$$
\begin{aligned}
E^{2}(z)= & \Omega_{m}(1+z)^{3}+\Omega_{k}(1+z)^{2}+ \\
& \left(1-\Omega_{m}-\Omega_{k}\right) \times \\
& {\left[A_{s}+\left(1-A_{s}\right)(1+z)^{3(1+B)(1+\alpha)}\right]^{\frac{1}{1+\alpha}} . }
\end{aligned}
$$

The GCG model corresponds to $B=0$, and the CG model corresponds to $B=0$ and $\alpha=1$.

2.1.1.9. Interacting $D E$ and $D M\left(I D E_{1}, I D E_{2}\right)$ - In typical cosmological models, including all of those discussed above, the dark matter and dark energy are assumed to evolve independently. However, some scalar field models can couple to the ordinary matter or dark matter (Amendola 2000, Farrar \& Peebles 2004). This kind of interaction of the dark sector can help resolve the coincidence problem. Such models have been widely studied (Zimdahl et al.|2001; Dalal et al.|2001, Tocchini-Valentini $\&$ Amendola 2002; Chimento et al. 2003; Caldera-Cabral et al. 2009 Bean et al.|2008 and references therein). In general, the interaction between the dark sector components affects observables. While some interaction models can mimic the expansion history of the $\Lambda \mathrm{CDM}$ model, the coupled dark energy model cannot in general mimic the expansion and growth of structure at the same time (Huterer et al. 2015).

In this paper, we consider the models proposed in Fay (2016), which have.

$$
\begin{gathered}
\bar{H}^{2} \propto \bar{\rho}_{m}+\bar{\rho}_{\mathrm{de}} \\
\bar{\rho}_{m}^{\prime}+3 \bar{\rho}_{m}=\bar{Q} / \bar{H} \\
\bar{\rho}_{\mathrm{de}}^{\prime}+3(1+\bar{w}) \bar{\rho}_{\mathrm{de}}=-\bar{Q} / \bar{H},
\end{gathered}
$$

where the bar denotes the interacting quantity, $\bar{Q}$ characterizes the interaction term, and the prime means the derivative with respect to $\ln a$. The particular models are specified by the equation of state of interaction dark energy $\bar{w}$, which together with $\rho_{\text {de }}$ and $\bar{H}$ determines $\bar{Q}$. We consider two models in this paper as detailed in Fay (2016):

$$
\begin{array}{cl}
\text { Model I }\left(I D E_{1}\right): & \bar{w}=\text { constant } \\
\text { Model II }\left(I D E_{2}\right): & \bar{w}=\bar{w}_{0}+\bar{w}_{1} \ln a,
\end{array}
$$

where $\bar{w}_{0}$ and $\bar{w}_{1}$ in Model II are constants.

In order to investigate the possibility of the interacting model to solve the tension of $\mathrm{H}_{0}$, we also consider a model without requiring it to mimic the expansion history as the $\Lambda \mathrm{CDM}$ model. This model has a interaction term proportional to the dark energy component $Q=\xi H \rho_{D E}$ with the coupling strength $\xi$. Note this model (hereafter $\mathrm{IDE}_{3}$ ) has the specific use for the $\mathrm{H}_{0}$ problem and not examined by the $p$-value test.

2.1.1.10. Weakly-coupled canonical scalar field (WCSF $1 \mathrm{D}$, $\left.W C S F_{2 \mathrm{D}}\right)$ - Similar to inflation, the late time acceleration of the universe can be explained by a scalar field with some potential function. Huang et al. (2011) introduced a three-parameter approximation of the equation of state $w\left(z ; \epsilon_{s}, \epsilon_{\infty}, \zeta_{s}\right)$ which allows trajectories from a 
wide class of scalar field potentials

$$
w=-1+\frac{2}{3}\left[\sqrt{\epsilon_{\infty}}+\left(\sqrt{\epsilon_{s}}-\sqrt{2 \epsilon_{\infty}}\right)\left[F\left(\frac{a}{a_{\mathrm{eq}}}\right)+\zeta_{s} F_{2}\left(\frac{a}{a_{\mathrm{eq}}}\right)\right]\right]^{2},
$$

where $\epsilon_{s}$ is the "slope parameter" defined as $\epsilon_{s} \equiv$ $\left.\epsilon_{V}\right|_{a=a_{\mathrm{eq}}}$ which measures the slope of the potential. $\epsilon_{\infty}$ is the "tracking parameter" which is defined from $\epsilon_{\infty} \equiv$ $\left.\epsilon_{V} \Omega_{\mathrm{de}}\right|_{a \rightarrow 0}$. The third parameter $\zeta_{s}$ captures the timedependence of $\epsilon_{V}$ as a higher order correction, which is poorly constrained by the current and forecasted data. Therefore we set it to be zero in the following discussion. The function $F(x)$ is defined as

$$
F(x)=\frac{\sqrt{1+x^{3}}}{x^{3 / 2}}-\frac{\ln \left(x^{3 / 2}+\sqrt{1+x^{3}}\right)}{x^{3}} .
$$

$a_{\text {eq }}$ is the scale factor of "matter-DE" equality which can be approximated by a fitting formula (Huang et al.|2011). In this paper, we discuss the one-parameter $\left(\epsilon_{\infty}=0\right)$ and two-parameter classes, identified as $\mathrm{WCSF}_{1 \mathrm{D}}$ and $\mathrm{WCSF}_{2 \mathrm{D}}$ respectively,

2.1.1.11. Holographic dark energy (HDE, ADE) - The holographic principle plays an important role in modern theoretical physics. In quantum gravity, the entropy of a system scales with its surface area instead of volume (see 't Hooft 1993; Susskind 1995, 't Hooft 2001, Bekenstein 1973, 1981, 1994, Hawking 1976 and references therein), which contradicts the prediction from effective field theory where the entropy is an extensive quantity. The reconciliation is suggested in Cohen et al. (1999) by introducing a relationship between the $U V$ and $I R$ cut-off and thus an energy bound. However, this approach fails to produce the current cosmic acceleration as pointed out in Hsu (2004). The solution of this problem is suggested in $\mathrm{Li}(2004)$ which is to use the future event horizon as the characteristic length scale. The resulting holographic dark energy model (HDE) has energy density

$$
\rho_{\mathrm{de}}=3 c^{2} M_{\mathrm{PL}}^{2} L^{-2},
$$

where $c$ is a numerical constant, $M_{\mathrm{PL}}=1 / \sqrt{8 \pi G}$ is the reduced Planck mass and $L$ is related to the characteristic length scale. The Friedmann equation can be written as

$$
E^{2}(z)=\frac{\Omega_{m}(1+z)^{3}}{1-\Omega_{\mathrm{de}}},
$$

where $\Omega_{\mathrm{de}}$ is determined through (Huang \& Li 2004, Zhang \& Wu 2007)

$$
\frac{d \Omega_{\mathrm{de}}}{d z}=-\Omega_{\mathrm{de}} \frac{1-\Omega_{\mathrm{de}}}{1+z}\left(1+\frac{2}{c} \sqrt{\Omega_{\mathrm{de}}}\right)
$$

Similar to HDE, Cai (2007) proposes the agegraphic dark energy model (ADE) motivated by the Karolyhazy relation (Karolyhazy 1966, Maziashvili 2007b a). The dark energy density is determined by a time scale $T$

$$
\rho_{\mathrm{de}}=3 n^{2} M_{\mathrm{PL}}^{2} T^{-2},
$$

where $n$ is a constant to parameterize uncertainties. The original ADE model applies the age of the universe as the time scale. However, in this model the agegraphic dark energy in fact never dominates the universe's dynamics (Wei \& Cai 2008). Therefore we consider the new agegraphic dark energy which uses the "conformal time" instead of the age of the universe. The solution is also expressed as a differential equation:

$$
\frac{d \Omega_{\mathrm{de}}}{d z}=-\Omega_{\mathrm{de}} \frac{1-\Omega_{\mathrm{de}}}{1+z}\left(3-\frac{2(1+z)}{n} \sqrt{\Omega_{\mathrm{de}}}\right) .
$$

Rather than using the length scale or time scale as the IR cut-off, Gao et al. (2009) propose another possibility: the length scale is given by the average radius of Ricci scalar curvature, $R^{1 / 2}$ (hereafter RDE). Unlike the HDE and ADE models, the Hubble parameter of this model can be expressed analytically, which is convenient in numerical solutions:

$E^{2}(z)=\frac{2 \Omega_{m}}{2-\alpha}(1+z)^{3}+\Omega_{k}(1+z)^{2}+\left(1-\Omega_{k}-\frac{2 \Omega_{m}}{2-\alpha}\right)(1+z)^{4-\frac{2}{\alpha}}$,

where $\alpha$ is a constant to be determined.

2.1.1.12. Quintessence scalar field model (QPL, QEX)Scalar fields can naturally arise in particle physics. They are also a simple generalization of the cosmological constant, and can play the role of dark energy. A quintessence field is such a scalar field, with standard kinetic energy and minimally coupling to gravity. In a spatially flat FRW universe, the evolution of a scalar field $\phi$ is governed by the Friedmann equation Eq. (3) and the Klein-Gordon equation

$$
\ddot{\phi}+3 H \dot{a}+\frac{d V}{d \phi}=0,
$$

where the dot denotes derivative with respect to cosmic time $t$. This model is completed by specifying the potential. In this paper, we consider two types of potential

$$
\begin{aligned}
\text { Model I }(Q P L) & : V \propto \phi^{-n} \\
\text { Model II }(Q E X): V & \propto \exp (-\lambda \phi),
\end{aligned}
$$

where the proportionality can be determined by the initial conditions, and $n$ and $\lambda$ are the free parameters. Model I was originally proposed by several authors (Ratra \& Peebles 1988, Peebles \& Ratra 1988; Caldwell et al. 1998). The solution from this model can alleviate the fine-tuning problem (Watson \& Scherrer 2003). Model II was first motivated by considering the consequences of an anomaly in the dilatation symmetry within particle physics (Wetterich 1988). This potential can give rise to accelerated expansion, and yields a scaling solution (Copeland et al. 1998; Barreiro et al. 2000) in which the energy density of dark energy is proportional to the matter.

2.1.1.13. QCD ghost dark energy - The QCD ghost dark energy (hereafter QCD) was proposed in Urban \& Zhitnitsky (2010a, 2009a b, 2010b); Ohta (2011). The key ingredient of this model is the Veneziano ghost field which is required to exist for the resolution of the $\mathrm{U}(1)$ problem in QCD (Witten 1979, Veneziano 1979, Rosenzweig et al. 1980, Kawarabayashi \& Ohta 1980; Kawarabayashi \& Ohta 1981; Ohta 1981). This field yields a vacuum energy density when it exists in a curved space or time-dependent background. In a flat FRW universe, the energy density of $\mathrm{DE}$ in this model is given by $\rho_{\mathrm{DE}}=\alpha H+\beta H^{2}$, where $\alpha$ and $\beta$ are constants (Cai et al. 2012). After a redefinition of the parameters, we 
can write the Friedmann equation of the QCD ghost dark energy model as:

$$
E^{2}(z)=\kappa+\sqrt{\kappa^{2}+\frac{\Omega_{m}(1+z)^{3}+\Omega_{r}(1+z)^{4}}{\gamma}},
$$

where $\kappa=\left(1-\left(\Omega_{m}+\Omega_{r}\right) / \gamma\right) / 2$. Therefore this model has one more free parameter $\gamma$ compared with the $\Lambda \mathrm{CDM}$ model.

\subsubsection{Modified gravity}

2.1.2.1. DGP cosmology - As a modified gravity theory, DGP model is proposed by Dvali et al. (2000) as a braneworld model where our universe is a 4-dimensional brane embedded in a 5-dimensional bulk. It differs from the RS braneworld model (Randall \& Sundrum 1999; Randall \& Sundrum 1999) by a curvature term on the brane. We follow the treatment of this model as detailed in Lombriser et al. (2009), wherein the Hubble parameter can be written as

$$
\begin{aligned}
E^{2}(z)= & \left(\sqrt{\Omega_{m}(1+z)^{3}+\Omega_{r}(1+z)^{4}+\Omega_{\Lambda}+\Omega_{r_{c}}}+\sigma \sqrt{\Omega_{r_{c}}}\right) \\
& +\Omega_{k}(1+z)^{2},
\end{aligned}
$$

where the density parameters have the same meaning as $\Lambda \mathrm{CDM}$ model. $\sigma=+1$ refers to the self-accelerating branch (sDGP) which has late time acceleration without a cosmological constant. $\sigma=-1$ is the normal branch (nDGP) where the DGP modifications slow the expansion rate. In the normal branch, a cosmological constant is then required to achieve late-time acceleration. Here

$$
\sqrt{\Omega_{r_{c}}}=\frac{1}{2 H_{0} r_{c}}=\sigma \frac{\Omega_{\mathrm{DGP}}}{2 \sqrt{1-\Omega_{k}}},
$$

where $\Omega_{\mathrm{DGP}}=1-\Omega_{m}-\Omega_{r}-\Omega_{k}-\Omega_{\Lambda}$, and $r_{c}$ is the crossover distance which governs the transition from $5 \mathrm{D}$ to $4 \mathrm{D}$ scalar-tensor gravity.

2.1.2.2. $f(R)$ gravity - $f(R)$ gravity is a non-trivial modification of GR which replaces the Ricci scalar in the Einstein-Hilbert action by a non-linear function. A detailed introduction of this theory is given in Sotiriou \& Faraoni (2010) and references therein. This model has the action

$$
S=\int d^{4} x \sqrt{-g}\left[\frac{R+f(R)}{2 \mu^{2}}+\mathcal{L}_{\mathrm{m}}\right],
$$

where $R$ is the Ricci scalar, $\mu^{2} \equiv 8 \pi G$ and $\mathcal{L}_{\mathrm{m}}$ is the Lagrangian of matter. This model is completed once the functional form of $f(R)$ is specified. Much attention has been paid to this class of models, both theoretically and observationally (Capozziello \& Fang 2002 , Nojiri \& Odintsov 2003; Capozziello et al. 2003; Cognola et al. 2005: Amendola et al. 2007b a; Hu \& Sawicki 2007; Starobinsky |2007; Li \& Barrow 2007; Zhao et al. 2011).

In this paper, we consider the designer model introduced by Song et al. (2007) and Lombriser et al. (2012) . This model mimics by construction the expansion history of the $\Lambda \mathrm{CDM}$ model. Imposing this constraint yields a form for $f(R)$ but also some freedom. Measurements of the growth of perturbations constrain the form $f(R)$ can take further. The form of $f(R)$ can be parameterized in terms of the Compton wavelength parameter

$$
B=\frac{f_{R R}}{1+f_{R}} R^{\prime} \frac{H^{\prime}}{H},
$$

evaluated at $B_{0} \equiv B(\ln a=0)$, where $f_{R} \equiv d f / d R$, $f_{R R} \equiv d^{2} f / d R^{2}$, and the prime denotes the derivative with respect to $\ln a$. This parameter indicates the modification of gravity, and $B_{0}=0$ recovers the standard gravity. The condition of stability requires $B_{0} \geq 0$ (Song et al. 2007, Sawicki \& Hu 2007, Lombriser et al. 2012).

2.1.2.3. $f(T)$ gravity - $f(T)$ gravity is based on the teleparallel equivalent of general relativity (Einstein 1928; Unzicker \& Case 2005, Hayashi \& Shirafuji 1979, Arcos \& Pereira 2004 Maluf 1994). A comprehensive review of this theory can be found in Cai et al. (2015). The key ingredient in this formulation is the torsion tensor which plays the role as Ricci tensor in general relativity. The natural extension of this formulation is to generalize the Lagrangian to be a function of $T$, which 2is the equivalent quantity of $R$ in general relativity and $f(R)$ gravity (Ferraro \& Fiorini 2007, 2008, Bengochea \& Ferraro 2009; Linder 2010). Unlike $f(R)$ gravity, the field equation in $f(T)$ theory is second-order rather than fourth-order, and this may cause pathological behaviors. In general, the corresponding action of $f(T)$ gravity is

$$
S=\frac{1}{16 \pi G} \int d^{4} x e[T+f(T)],
$$

where $e=\operatorname{det}\left(\mathrm{e}_{\mu}^{\mathrm{A}}\right)=\sqrt{-\mathrm{g}}$, and $e_{\mu}^{A}$ is the vierbein fields. Note that this theory gives $\Lambda$ CDM model when $f(T)=$ constant.

In a flat FRW universe, the resulting Friedmann equation can be written as (Nesseris et al.]2013)

$$
E^{2}(z)=\Omega_{m}(1+z)^{3}+\Omega_{r}(1+z)^{4}+\left(1-\Omega_{m}-\Omega_{r}\right) y(z),
$$

where $y(z)$ is a function of redshift $z$ dependent on the particular $f(T)$ model. In this paper, we consider the following specific $f(T)$ models from the literature.

Model I $\left(f(T)_{P L}\right)$ : the power-law model (Bengochea \& Ferraro 2009)

$$
f(T)=\alpha(-T)^{b},
$$

where $\alpha$ and $b$ are parameters. The function in the Friedmann equation is

$$
y(z)=E^{2 b}(z)
$$

Model II $\left(f(T)_{E x p 1}\right)$ : the exponential model as $f(R)$ gravity (Nesseris et al. 2013, Linder 2009)

$$
f(T)=\alpha T_{0}\left(1-e^{-T /\left(b T_{0}\right)}\right),
$$

with $\alpha$ and $b$ are parameters and

$$
y(z)=\frac{1-\left(1+\frac{2 E^{2}}{b}\right) e^{-E^{2} / b}}{1-\left(1+\frac{2}{b}\right) e^{-1 / b}} .
$$

Model III $\left(f(T)_{\operatorname{Exp} 2}\right)$ : the exponential model proposed by Linder (2009)

$$
f(T)=\alpha T_{0}\left(1-e^{-\sqrt{T / T_{0}} / b}\right),
$$


where $\alpha$ and $b$ are parameters, and

$$
y(z)=\frac{1-\left(1+\frac{E}{b}\right) e^{-E / b}}{1-\left(1+\frac{1}{b}\right) e^{-1 / b}} .
$$

Model IV $\left(f(T)_{\tanh }\right)$ : the hyperbolic-tangent model proposed by Wu \& Yu (2011)

$$
f(T)=\alpha(-T)^{n} \tanh \frac{T_{0}}{T},
$$

where $\alpha$ and $n$ are model parameters. We also obtain

$$
y(z)=E^{2(n-1)} \frac{2 \operatorname{sech}^{2}\left(\frac{1}{E^{2}}\right)+(1-2 n) E^{2} \tanh \left(\frac{1}{E^{2}}\right)}{2 \operatorname{sech}^{2}(1)+(1-2 n) \tanh (1)} .
$$

This model is different from the previous 3 models in that it cannot return to $\Lambda \mathrm{CDM}$ for any value of the parameters.

Another model we do not consider here is that of Bamba et al. (2011):

$$
\left.f(T)=\alpha T_{0} \sqrt{(} \frac{T}{q T_{0}}\right) \ln \left(\frac{q T_{0}}{T}\right),
$$

where $\alpha$ and $q$ are model parameters. The Hubble parameter in this model is independent from $\alpha$ or $q$, and it coincides with the flat sDGP model without cosmological constant. Therefore the analysis of sDGP model is equivalent to this model. This model has different dynamical behavior than sDGP which is represented by the perturbation equation (Nesseris et al. 2013). However, we do not consider this model here, as it is already ruled out by the expansion and CMB data for the same reasons sDGP is.

2.1.2.4. Galileon cosmology: Tracker solution (GAL)Galileon theory is a scalar field model introduced in Nicolis et al. (2009); Deffayet et al. (2009a b). This model is inspired by the DGP model and its ability to produce the current acceleration without dark energy. This model is invariant under the Galileon symmetry in the Minkowski space-time, and keeps the field equation to be second order. The Galileon model considered in this paper is detailed in Nesseris et al. (2010), de Felice \& Tsujikawa (2010), de Felice et al. (2011), and de Felice \& Tsujikawa (2011). For numerical purposes, we consider the tracker solution, which has the Hubble parameter (hereafter $G A L$ ):

$$
\begin{aligned}
& E^{2}(z)=\frac{1}{2} \Omega_{k}(1+z)^{2}+\frac{1}{2} \Omega_{m}(1+z)^{3}+\frac{1}{2} \Omega_{r}(1+z)^{4} \\
& +\sqrt{\Omega_{g}+\frac{(1+z)^{4}}{4}\left[\Omega_{m}(1+z)+\Omega_{k}+\Omega_{r}(1+z)^{2}\right]^{2}}(, 60)
\end{aligned}
$$

where $\Omega_{g}=1-\Omega_{m}-\Omega_{k}-\Omega_{r}$. Note that this model has the same parameters as $\Lambda \mathrm{CDM}$ model.

2.1.2.5. Kinetic gravity braiding model (KGBM, $K G B M_{n=1}$ ) - The kinetic braiding model is inspired from Galileon model (Deffayet et al. 2010), which introduces the extended self-interaction term $G(\phi, X) \square \phi$ minimally coupled to gravity, where $G(\phi, X)$ is a function of $\phi$ and $X$ with $X=-g^{\mu \nu} \nabla_{\mu} \phi \nabla_{\nu} \phi / 2$. This model is further generalized in Kimura \& Yamamoto (2011) as $G(\phi, X) \propto X^{n}$ and the original model corresponds to $n=1$. In a flat FRW universe, the Friedmann equation is

$$
E^{2}(z)=\left(1-\Omega_{m}-\Omega_{r}\right) E^{-\frac{2}{2 n-1}}+\Omega_{m}(1+z)^{3}+\Omega_{r}(1+z)^{4} .
$$

For $n=1$, the expansion in this model is the same as the tracking solution in Galileon model Eq. (60). If $n$ is taken as an arbitrary parameter, the expansion is equivalent to the power-law $f(T)$ theory Eq. (52), despite the fact that these two models have different physical mechanisms. However, the KGBM and $f(T)_{\mathrm{PL}}$ growth rate predictions do differ, which can be used to distinguish them.

\subsection{Data sets}

We now discuss the data sets we use in this paper. We will consider various combinations of the following five data sets.

\subsubsection{BAO data}

The BAO data arise from the measurements of the correlation function or power spectrum of large scale structure tracers. As an absolute distance measurement, the determination of the BAO scale is based on an fiducial cosmology, which translates the angular and redshift separations to comoving distances. In an anisotropic analysis, the measurement of the BAO scale constrains the comoving angular diameter distance $D_{M}(z)$ and the Hubble parameter $H(z)$ through

$$
D_{M}(z) / r_{d}=\alpha_{\perp} D_{M, \text { fid }} / r_{d, \text { fid }}
$$

and

$$
D_{H}(z) / r_{d}=\alpha_{\|} D_{H, \text { fid }} / r_{d, \text { fid }},
$$

where $D_{H}(z)=c / H(z)$ and $r_{d}$ is the sound horizon at the drag epoch $z_{d}$ when photons and baryons decouple:

$$
r_{d}=\int_{z_{d}}^{\infty} \frac{c_{s}(z)}{H(z)} d z
$$

with the sound speed in the photon-baryon fluid $c_{s}(z)=$ $3^{-1 / 2} c\left[1+\frac{3}{4} \rho_{b}(z) / \rho_{\gamma}(z)\right]^{-1 / 2}$. The subscript "fid" refers to the quantity in the assumed fiducial model. $\alpha_{\perp}$ and $\alpha_{\|}$ are the ratios of the distances perpendicular and parallel to the line of sight.

An isotropic BAO analysis can be interpreted as constraining an effective distance that is a combination of $D_{M(z)}$ and $D_{H}(z)$ (Eisenstein et al. 2005)

$$
D_{V}=\left[z D_{H}(z) D_{M}^{2}(z)\right]^{1 / 3}
$$

through

$$
D_{V}(z) / r_{d}=\alpha D_{V, \text { fid }}(z) / r_{d, \text { fid }},
$$

where $\alpha$ is the ratio of the BAO scale to that predicted by the fiducial model.

The measurements of BAO come from galaxy surveys and Lyman $\alpha$ Forest (LyaF) surveys. In our calculation, the data adopted are taken from 6dFGS (Beutler et al. 2011), the SDSS main galaxy sample (MGS, Ross et al. 2015), BOSS galaxies (Anderson et al. 2014), the BOSS LyaF auto-correlation (Delubac et al. 2015), and the BOSS LyaF cross-correlation (Font-Ribera et al. 2014). The likelihood calculations of these data are the same as Aubourg et al. (2015) (as summarized in their 


\begin{tabular}{|c|c|c|c|c|c|c|c|}
\hline Model & Acronym & parametera $^{a}$ & Section & Not in tension with: & $f \sigma_{8}$ & $H_{0}$ & LyaF BAO \\
\hline Flat $\Lambda$ CDM model & $\Lambda \mathrm{CDM}$ & $\Omega_{m}, h, \sigma_{8}$ & 2 & & Yes & No & No \\
\hline Non-flat $\Lambda$ CDM model & $o \Lambda \mathrm{CDM}$ & $\Omega_{m}, \Omega_{k}, h, \sigma_{8}$ & 2 & & Yes & No & No \\
\hline Constant parameterization & $\mathrm{XCDM}$ & $w$ & 2.1 .1 .1 & & Yes & No & No \\
\hline CPL parameterization & CPL & $w_{0}, w_{1}$ & 2.1 .1 .1 & & Yes & No & No \\
\hline JBP parameterization & JBP & $w_{0}, w_{1}$ & 2.1 .1 .1 & & Yes & No & No \\
\hline Linear parameterization & $w_{\text {Linear }}$ & $w_{0}, w_{1}$ & 2.1 .1 .1 & & Yes & No & No \\
\hline Pseudo-Nambu Goldstone Boson & PNGB & $w_{0}, F$ & 2.1 .1 .2 & & Yes & No & No \\
\hline Cassimir effect & $\mathrm{CE}$ & $\Omega_{c}$ & 2.1 .1 .3 & & Yes & No & No \\
\hline Cardassian ansatz & $\mathrm{CA}$ & $q, n$ & 2.1 .1 .4 & & Yes & No & No \\
\hline Early dark energy & EDE & $w_{0}, \Omega_{\mathrm{de}}^{\mathrm{e}}$ & 2.1 .1 .5 & & Yes & No & No \\
\hline Slow roll dark energy & SR & $\delta w_{0}$ & 2.1 .1 .6 & & Yes & No & No \\
\hline Polynomial CDM & PolyCDM & $\Omega_{1}, \Omega_{2}$ & 2.1 .1 .7 & & Yes & No & Yes \\
\hline Logarithmic Hubble parameter & HLG & $\beta$ & 2.1 .1 .7 & & Yes & No & No \\
\hline Chaplygin gas & CG & $A_{s}$ & 2.1 .1 .8 & & Yes & No & No \\
\hline Generalized Chaplygin gas & GCG & $A_{s}, \alpha$ & 2.1 .1 .8 & & Yes & No & No \\
\hline Modified Chaplygin gas & MCG & $A_{s}, \alpha, B$ & 2.1 .1 .8 & & Yes & No & No \\
\hline Interacting DE (model I) & $\mathrm{IDE}_{1}$ & $\bar{w}$ & 2.1 .1 .9 & & Yes & No & No \\
\hline Interacting DE (model II) & $\mathrm{IDE}_{2}$ & $\bar{w}_{0}, \bar{w}_{1}$ & 2.1 .1 .9 & & Yes & No & No \\
\hline Weakly-coupled scalar field (1D) & $\mathrm{WCSF}_{1 D}$ & $\epsilon_{s}$ & 2.1 .1 .10 & & Yes & No & No \\
\hline Weakly-coupled scalar field (2D) & $\mathrm{WCSF}_{2 D}$ & $\epsilon_{s}, \epsilon_{\infty}$ & 2.1 .1 .10 & & Yes & No & No \\
\hline Holographic dark energy & HDE & $c$ & 2.1 .1 .11 & & Yes & No & No \\
\hline Agegraphic dark energy & $\mathrm{ADE}$ & $n$ & 2.1 .1 .11 & & Yes & No & No \\
\hline Ricci scalar dark energy & $\mathrm{RDE}$ & $\alpha$ & 2.1 .1 .11 & & No & No & No \\
\hline Quintessence: Power-law potential & QPL & $n$ & 2.1 .1 .12 & & Yes & No & No \\
\hline Quintessence: Exponential potential & QEX & $\lambda$ & 2.1 .1 .12 & & Yes & No & No \\
\hline QCD Ghost dark energy & QCD & $\gamma$ & 2.1 .1 .13 & & No & No & Yes \\
\hline DGP cosmology & DGP & - & 2.1 .2 .1 & & No & No & No \\
\hline$f(R)$ gravity $(\mathrm{k}=0.1)$ & $f(R)_{1}$ & $B_{0}$ & 2.1 .2 .2 & & Yes & No & No \\
\hline$f(R)$ gravity $(\mathrm{k}=0.02)$ & $f(R)_{2}$ & $B_{0}$ & 2.1 .2 .2 & & Yes & No & No \\
\hline$f(T)$ gravity (model I) & $f(T)_{P L}$ & $b$ & 2.1 .2 .3 & & Yes & No & No \\
\hline$f(T)$ gravity (model II) & $f(T)_{\operatorname{Exp} 1}$ & $b$ & 2.1 .2 .3 & & Yes & No & No \\
\hline$f(T)$ gravity (model III) & $f(T)_{\operatorname{Exp} 2}$ & $b$ & 2.1 .2 .3 & & Yes & No & No \\
\hline$f(T)$ gravity (model IV) & $f(T)_{\text {tanh }}$ & $n$ & 2.1 .2 .3 & & No & No & No \\
\hline Galileon cosmology: tracker solution & GAL & - & 2.1 .2 .4 & & No & Yes & No \\
\hline Kinetic gravity braiding model & KGBM & $n$ & 2.1 .2 .5 & & Yes & No & No \\
\hline Kinetic gravity braiding model with $n=1$ & $\mathrm{KGBM}_{n=1}$ & - & 2.1 .2 .5 & & No & No & No \\
\hline
\end{tabular}

${ }^{\mathrm{a}}$ For models other than $\Lambda \mathrm{CDM}$ and $o \Lambda \mathrm{CDM}$ model, only the different parameters are shown

Table 1

Models considered in the paper, including their names, parameters and section in the paper where they are introduced. The last three columns also present their performance and the tension with the linear growth data, local $\mathrm{H}_{0}$ measurement and $\mathrm{BAO}$ measurements from LyaF. For LyaF BAO, we classify the model as "Yes" if the $p$-value difference $\Delta p$ is smaller than 0.1 and "No" when $\Delta p$ is larger than 0.1 when the dataset is added. For $\mathrm{H}_{0}$ and linear growth, the tension is assumed as existed if the model does not perform much better than $\Lambda \mathrm{CDM}$ model.

Table II), and we refer the readers to that paper for more details.

\subsubsection{Linear growth data}

The growth of structure is an important probe to test dark energy and modified gravity models, especially when geometrical measurements are not able to distinguish them from $\Lambda$ CDM. For scales well within the Hubble radius, the growth of structure is governed by the equation

$$
\ddot{\delta}+2 H \dot{\delta}-4 \pi G \rho_{m} \delta=0,
$$

where the dots are derivatives with respect to time, and $\delta \equiv \delta \rho_{m} / \rho_{m}$ is the matter density contrast. Note that this equation should be modified accordingly in the modified gravity theories, or when the interaction between dark energy and dark matter is taken into account. Examples can be found in Fay (2016); Tsujikawa (2007).

Here we consider measurements of this growth rate from redshift space distortions (Kaiser 1987; Scoccimarro 2004). This measurement of the growth is often represented by $f \sigma_{8}(z)$, where $f \equiv d \ln D / d \ln a$, with $D(z) \equiv$ $\delta(z) / \delta(0)$, and $\sigma_{8}(z)=\sigma_{8} D(z)$ is the power spectrum amplitude. The current value of $\sigma_{8}$ is a parameter to be fit, $f$ varies according to the cosmological model, and their combination is the observable that can be extracted from redshift space distortions. We calculate $f$ starting from initial conditions at $z \approx 30$, when the universe is dominated by matter and (for the models considered here) modified gravity effects have not become significant, and thus we have $\delta \propto a$ (Bertschinger \& Zukin 2008 Rapetti et al. 2013). The data we used in the calculation include measurements from 6dFGRS (Beutler et al. 2012), SDSS galaxy (Samushia et al. 2012) and BOSS CMASS galaxy (Beutler et al. 2014) samples, as shown in Figure 1. These measurements span a redshift range of $0.1 \lesssim z \lesssim 0.7$ with limited overlap in the target samples, making them independent data points. Specifically, the 6dFGRS survey is a Southern sky program, with a median redshift of $z \sim 0.07$. The Samushia et al. (2012) data measure clustering for galaxies in the SDSSI/11 Legacy survey, with median redshift of $z \sim 0.37$. These galaxies were also included in the overall target sample for the BOSS data analyzed in Beutler et al. 
(2014), but only $1.5 \%$ of CMASS targets were obtained from legacy SDSS data. This low fraction is due to the difference in redshift of the two samples, with CMASS probing structure at $z \sim 0.55$, and that $1 / 3$ of the BOSS footprint covers new area not mapped in SDSS. We note that the linear growth at this redshift was also measured by Reid et al. (2012); Chuang et al. (2013), the results are in good agreement with the one used in our analysis and the latter has better accuracy due to the increased survey area (Beutler et al. 2014) . These data at $z \gtrsim 0.1$ have been superceded by more recent analysis of the completed BOSS dataset (Alam et al. 2016), published after this work was in an advanced stage. The error bars on $f \sigma_{8}(z)$ are $\sim 10-20 \%$ smaller in Alam et al. (2016) than those used here, but the impact of replacing our current data with these new data would not significantly change our results.

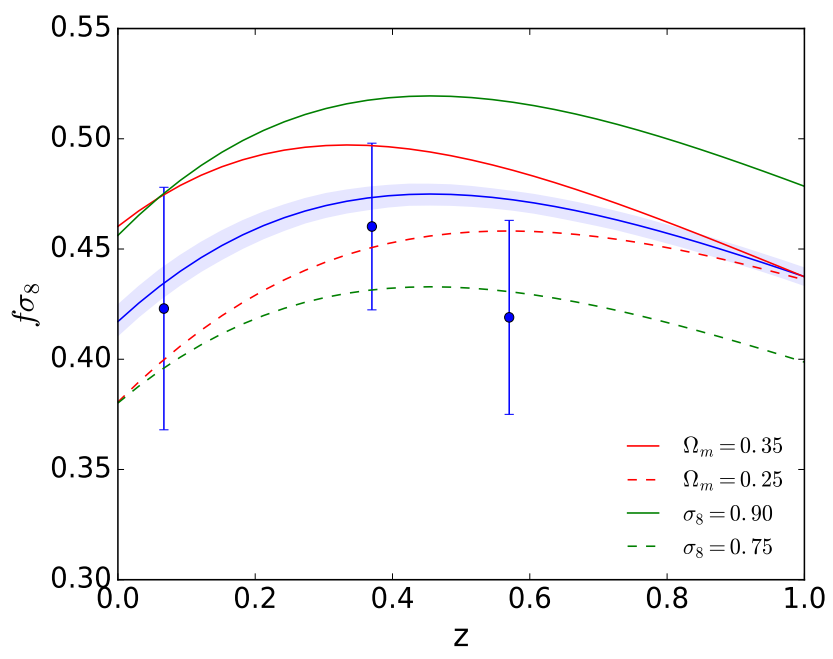

Figure 1. The linear growth data $f \sigma_{8}$ as a function of $z$. The solid blue line corresponds to the best-fit flat $\Lambda \mathrm{CDM}$ model from all the datasets, while the shaded region is the $1 \sigma$ error. Green and red lines represent the effect of the parameter $\Omega_{m}$ and $\sigma_{8}$ respectively (other parameters are kept fixed at the best-fit values).

\subsubsection{Cosmic Microwave Background data}

The Cosmic Microwave Background measurements contain important cosmological information. In this pa- per we focus on the expansion history and linear perturbation of the universe; therefore, we can use an approximation that avoids computation of the full $\mathrm{CMB}$ power spectrum for each model evaluation. Therefore we adopt the same strategy as Aubourg et al. (2015) and compress the CMB measurements to variables related to the expansion and linear growth. The geometrical aspect of this CMB compression is represented by the distance scale $D_{M}(1090) / r_{d}$ (a BAO measurement at $z=1090), \Omega_{m} h^{2}$ and $\Omega_{b} h^{2}$, the matter and baryon fractions of the universe, which determine the absolute length of the $\mathrm{BAO}$ ruler.

For our work, we quantify the amplitude of perturbations using $\sigma_{8}(z=30)$, the amplitude of the matter power spectrum at the initial epoch of the perturbation Eq. (67). Using this quantification of $\sigma_{8}$ allows us to avoid integrating the growth equation from $z \sim 1090$ to low redshift for every set of model parameters. $z \sim 30$ is after the point at which radiation matters to the expansion but before any of our dark energy or modified gravity models start to deviate from $\Lambda \mathrm{CDM}$, so it is a convenient starting point.

The compressed data vector for the CMB is then:

$$
\mathbf{v}=\left(\begin{array}{c}
\Omega_{b} h^{2} \\
\Omega_{m} h^{2} \\
D_{M}(1090) / r_{d} \\
\sigma_{8}(30)
\end{array}\right)
$$

In this paper, we use the publicly available base_Alens chains with the planck_lowl_lowLike dataset from the Planck dataset with low-l WMAP polarization (Planck Collaboration et al. 2014 ${ }^{3}$. Note that in the computation of $\sigma_{8}(30)$ for each sample in the Planck chains, we start from the late time variables in a Planck cosmology instead of the early time variables. This is only for the numerical considerations, and the accuracy compared from camb (Lewis \& Bridle 2002; Lewis et al. 2000) is found to be better than $1 \%$.

The resulting compression of the CMB data is represented by a simple Gaussian distribution with mean of the data vector

$$
\mu_{\mathbf{v}}=\left(\begin{array}{c}
0.02245 \\
0.1393 \\
94.27 \\
0.03448
\end{array}\right)
$$

and its covariance

$$
C_{\mathbf{v}}=\left(\begin{array}{cccc}
1.29 \times 10^{-7} & -6.04 \times 10^{-7} & 1.43 \times 10^{-5} & 3.45 \times 10^{-8} \\
-6.04 \times 10^{-7} & 7.55 \times 10^{-6} & -3.41 \times 10^{-5} & -2.36 \times 10^{-7} \\
1.43 \times 10^{-5} & -3.41 \times 10^{-5} & 4.24 \times 10^{-3} & -6.60 \times 10^{-7} \\
3.45 \times 10^{-8} & -2.36 \times 10^{-7} & -6.60 \times 10^{-7} & 2.20 \times 10^{-7}
\end{array}\right)
$$

For more details regarding this type of compression of the $\mathrm{CMB}$ data, we refer the readers to Aubourg et al.

\footnotetext{
3 The Planck 2015 chains (Planck Collaboration et al. 2015a)
} are not available at this time.
(2015).

\subsubsection{Supernovae data}

Type-Ia supernovae (SNe) are standardizable candles that can be used to probe the expansion history of the 
universe by measuring the luminosity distance as a function of redshift. In this paper, we use the Joint Lightcurve Analysis (JLA) sample (Betoule et al. 2014), which is constructed from the SDSS-II Supernova Survey (Sako et al. 2014) and the Supernova Legacy Survey (SNLS) 3year data set (Conley et al. 2011) combined with several samples of low redshift SNe. For simplicity, we here use the compressed representation of the relative distance constraints rather than the full cosmomc module. The compressed data is described by a vector in 31 redshift bins, and a covariance matrix. Note that absolute luminosity of $\mathrm{SNe}$ is considered uncertain and therefore in our analysis we marginalize over the fiducial absolute magnitude.

\subsubsection{Hubble constant $H_{0}$}

The Hubble constant $\left(\mathrm{H}_{0}\right)$ measures the local expansion of the universe. The CMB measurements depend on early universe conditions and about the integral of light propagation across the Universe. Therefore, the CMBinferred measurement of $H_{0}$ is model dependent, so the comparison with the local measurement is a test of the standard cosmological model. In this paper, we employ the analysis of Riess et al. (2016), which yields a $2.4 \%$ determination of the quantity

$$
H_{0}=73.24 \pm 1.74 \mathrm{kms}^{-1} \mathrm{Mpc}^{-1} .
$$

This value is $3.4 \sigma$ higher than the result from Planck data, and we will see its effect on the goodness of fit of the cosmological data in detail below.

\section{METHODOLOGY OF GOODNESS OF FIT}

As mentioned earlier in the introduction, one of the aims in this paper is to evaluate the goodness of fit for each model introduced in Section 2.1. Doing so will allow us to eliminate models that are incompatible with the data.

Evaluating a $p$-value requires the choice of a summary metric for the data. For a summary metric, we choose a $\chi^{2}$-like global statistic. For linear models and Gaussian uncertainties, the distribution of this statistic is just the $\chi^{2}$ distribution with $N-M$ degrees of freedom, where $N$ is the number of measurements and $M$ is the number of linear parameters. In such a case, the $p$-value for a given set of data, given that we have used the data to fit the parameters of the model, is trivial to calculate. However, the models described above do not make predictions that vary linearly with the model parameters, and the uncertainties in the data are not always modeled as Gaussian. The non-Gaussianity mainly comes from the LyaF, BOSS CMASS and SDSS MGS BAO measurements. Thus, we will estimate the $p$-value through a Monte Carlo experiment in which for a large number of simulated data sets drawn from the model we explicitly fit the model to the data and then calculate the $\chi^{2}$ statistic. In this procedure, fitting the model to each simulated data set effectively penalizes models which have extra degrees of flexibility that are relevant to the observed data.

We do not attempt in this investigation to select between models whose $p$-values are sufficiently compatible with the data (i.e. around $p \sim 0.05$ or better). A number of well-developed techniques exist for model selection, including the evaluation of the Bayes Factor and other simpler techniques such as the Akaike Information Criterion
(AIC, Akaike 1974), defined as $\mathrm{AIC}=-2 \mathcal{L}+2 k$, where $\mathcal{L}$ is the maximum likelihood and $k$ is the number of parameters, and the Bayesian Information Criterion (BIC, Schwarz et al. 1978) defined as $\mathrm{BIC}=-2 \mathcal{L}+k \ln N$, where $N$ is the number of data points. Our experiments with the latter two criteria indicate that they yield in practice for our problem similar results to comparing $p$-values. In the test with information criterion, the model with lower values of AIC or BIC is favored, and only the relative value between different models is of interest (Liddle 2004). For instance, a difference of 2 for BIC is considered as positive evidence, and of 6 or more as strong evidence against the model with larger value (Liddle 2004). A similar rule is also applicable to the outcome of AIC.

To calculate the $p$-value, in detail we perform the following steps:

1. For a given dataset and model, we find the maximum likelihood $\mathcal{L}$ with respect to the model parameters $\theta$. We take the best-fit of the parameters $\hat{\theta}$ as our point estimate of the true but unknown parameters $\Theta$. For linear models and Gaussian uncertainties, the quantity $-2 \ln \mathcal{L}(\hat{\theta})$ has a $\chi^{2}$ distribution, and we will refer to it below as " $\chi_{\min }^{2}$." In general, either $\mathcal{L}(\hat{\theta})$ or $\chi_{\min }^{2}$ is a global measure of how different the model and the data are.

2. We generate an ensemble of simulated data sets (specifically, the SN and BAO distances, compressed CMB parameters, $H_{0}$ measurements, and $\left.f \sigma_{8}\right)$ predicted under the model with the estimated $\hat{\theta}$, and assuming the estimated uncertainties (which in some cases are non-Gaussian).

3. For each simulated data set, we find the maximum likelihood $\mathcal{L}$ under the model while varying the parameters.

4. The $p$-value is defined as the fraction of the simulated data sets with smaller values of $\mathcal{L}(\hat{\theta})$ than the data.

The resulting $p$-value then reflects how infrequently we expect data with lower $\mathcal{L}(\hat{\theta})$ or higher $\chi^{2}$, given the model, if the model under consideration is correct.

As a side note, a more robust test of the model acceptability might let $\hat{\theta}$ vary over an acceptable range in the above simulations. Since the parameters of our models are generally well-constrained, we do not explore the effect of doing so.

Fig. 2 shows the application of this method to the spatially flat $\Lambda$ CDM model, which is the simplest model. The vertical lines show $\chi_{\min }^{2}$ for eight different combinations of the data. The curves show the cumulative probability of finding a lower $\chi_{\min }^{2}$ estimated using the simulations described above. The points indicate the position of the real data in this distribution. Adding more data raises $\chi_{\min }^{2}$, but also raises the expected distribution. In this plot the vertical axis is 1 minus the $p$-value.

The $p$-values range from 0.15 to about 0.01 depending on the data combination. That is, all data combinations experience a slight tension, and some of them are fairly severe, but none of them invalidate the underlying 
model at a significance comparable to the ordinarily desired 5- $\sigma$ criterion. In general, adding $H_{0}$ always lowers the $p$-value, due to the well-known tension between $H_{0}$ and other data sets. The lowest $p$-value among our data combinations is achieved by combining $\mathrm{BAO}, \mathrm{CMB}$, and $H_{0}$. Adding $f \sigma_{8}$ or $\mathrm{SNe}$ always raises the $p$-value; this rise results because adding these data effectively dilutes the impact of the $H_{0}$ tension on the global $\chi_{\min }^{2}$ statistic.

In detail, the addition of $H_{0}$ increases the $\chi_{\min }^{2}$ by at least 5.5. This is consistent with previous results reported by the Planck team (Planck Collaboration et al. 2014, 2015a) and Riess et al. (2016).

Fig. 3 shows the result with the addition of spatial curvature $\Omega_{k}$. The values of $\chi_{\min }^{2}$ from each real data set is smaller than in Fig. 2, which is consistent with expectation due to a small but non-zero curvature. The cumulative probability from the random ensemble is also shifted to the left because of the increased flexibility of the model. The amounts of the shift are not far from what one would expect for a $\chi^{2}$ distribution with one fewer degree of freedom. The resulting $p$-values are similar to those found for the spatially flat $\Lambda \mathrm{CDM}$ model; in some data combinations the data is a slightly greater outlier than for the flat model.

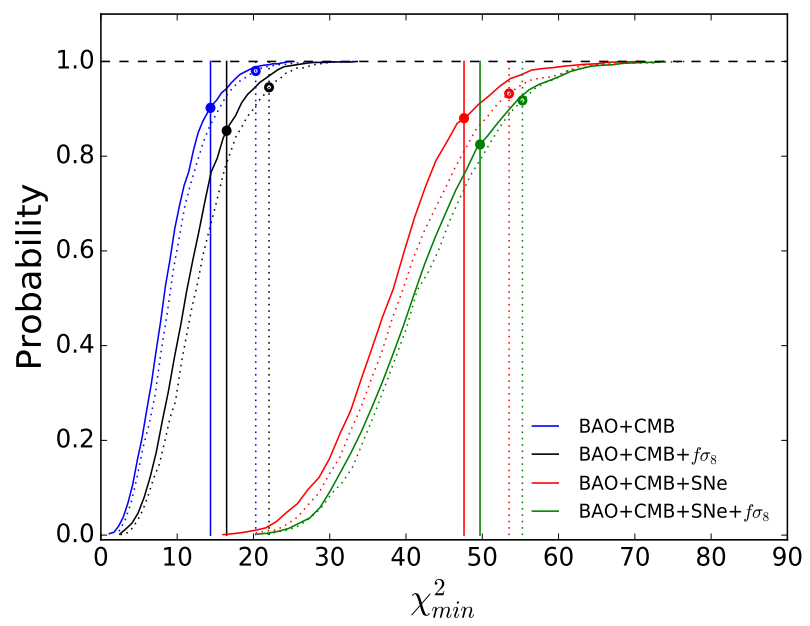

Figure 2. The $p$-value test of the flat $\Lambda \mathrm{CDM}$ model with different data combinations. The vertical lines correspond to the $\chi_{\min }^{2}$ (corresponding to the maximum likelihood $\mathcal{L}_{\max }$ ) from the real observations. The curved lines correspond to the cumulative probability from the distribution of $\mathcal{L}_{\max }$ from the random ensemble, which has one thousand realizations in this figure. The intersection points represented by the dots correspond to $1-p$, where $p$ is the probability under the model of observing data with a lower value of the maximum likelihood than the actual data. The dotted lines and empty dots correspond to the datasets plus the local Hubble constant $H_{0}$.

We apply this method to the models introduced in Section 2.1 and compare the inferred $p$-value from each data set. Figures 4 and 5 summarize the results of the $p$-value from different data combinations, in particular focusing on the effects of $H_{0}$ and linear growth.

In the top panel of Figure 4 all of the data is included. In the bottom panel of Figure 4 we exclude $H_{0}$ measurements. In the top panel of Figure 5 we exclude $f \sigma_{8}$ data.

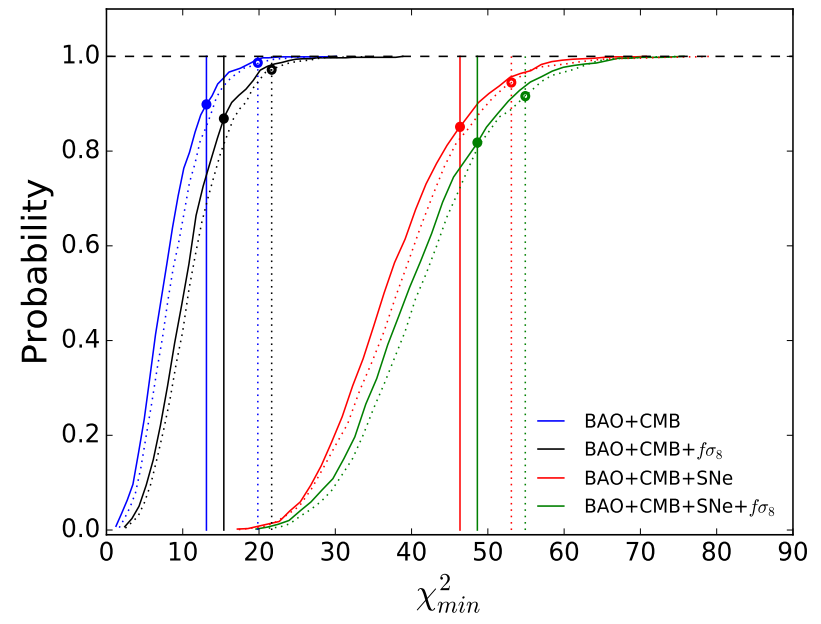

Figure 3. Similar to Fig 2 but for $\Lambda \mathrm{CDM}$ with spatial curvature $\Omega_{k}$.

In the bottom panel of Figure 5 we exclude both $\mathrm{H}_{0}$ and $f \sigma_{8}$. In these figures, the red points indicate modified gravity models.

The bottom of Figure 5 shows that even just with $\mathrm{BAO}, \mathrm{CMB}$, and $\mathrm{SNe}$ there are a handful of models fully incompatible with the data: RDE, GAL, and sDGP. Although GAL and sDGP are occasionally used as examples for which $f \sigma_{8}$ can yield extra information (because they are modified gravity models), in fact these particular examples are already strongly ruled out without it. The rest of the models exist between about $p \sim 0.1$ and 0.25 - they mostly have some mild tension with the data, but nothing alarming. The $f(R)$ models we consider here do not appear in this figure, because they yield identical expansion rate predictions to $\Lambda \mathrm{CDM}$ by construction.

On the top of Figure 5 we see the effect of adding back in $\mathrm{H}_{0}$. This makes all of the fits less compatible with the data, quite substantially, to $p \sim 0.03-0.13$. Given that this is a global statistic, it is indicative of how difficult that single data point is to fit. The Casimir Effect (CE) model and a handful of other models suffer the tension less than others, though not by actually fitting the $\mathrm{H}_{0}$ much better (see Section 4.2). Again, the $f(R)$ models do not appear.

On the bottom of Figure 4 we see the effect of adding back in $f \sigma_{8}$ (but not $\mathrm{H}_{0}$ ). $f \sigma_{8}$ fully rules out $f(T)_{\text {tanh }}$ and $\mathrm{KGBM}_{n=1}$ and furthermore makes KGBM and QCD decidedly less well favored. Notably, for a number of modified gravity models $(f(R)$ and the exponential and power law $f(T)$ models) it has virtually no effect on their compatibility with the data.

On the top of Figure 4 we see the full data combination. The QCD, RDE, GAL, $\mathrm{KGBM}_{n=1}, f(T)_{\text {tanh }}$, and sDGP models are all now fully ruled out. Note that in the way we have quantified goodness of fit, many models have higher $p$-values than without $f \sigma_{8}$ - this result means that $f \sigma_{8}$ 's large error bars are diluting the goodness of fit, possibly because they are overestimated but equally plausibly because they lie closer to most of these models by accident. 

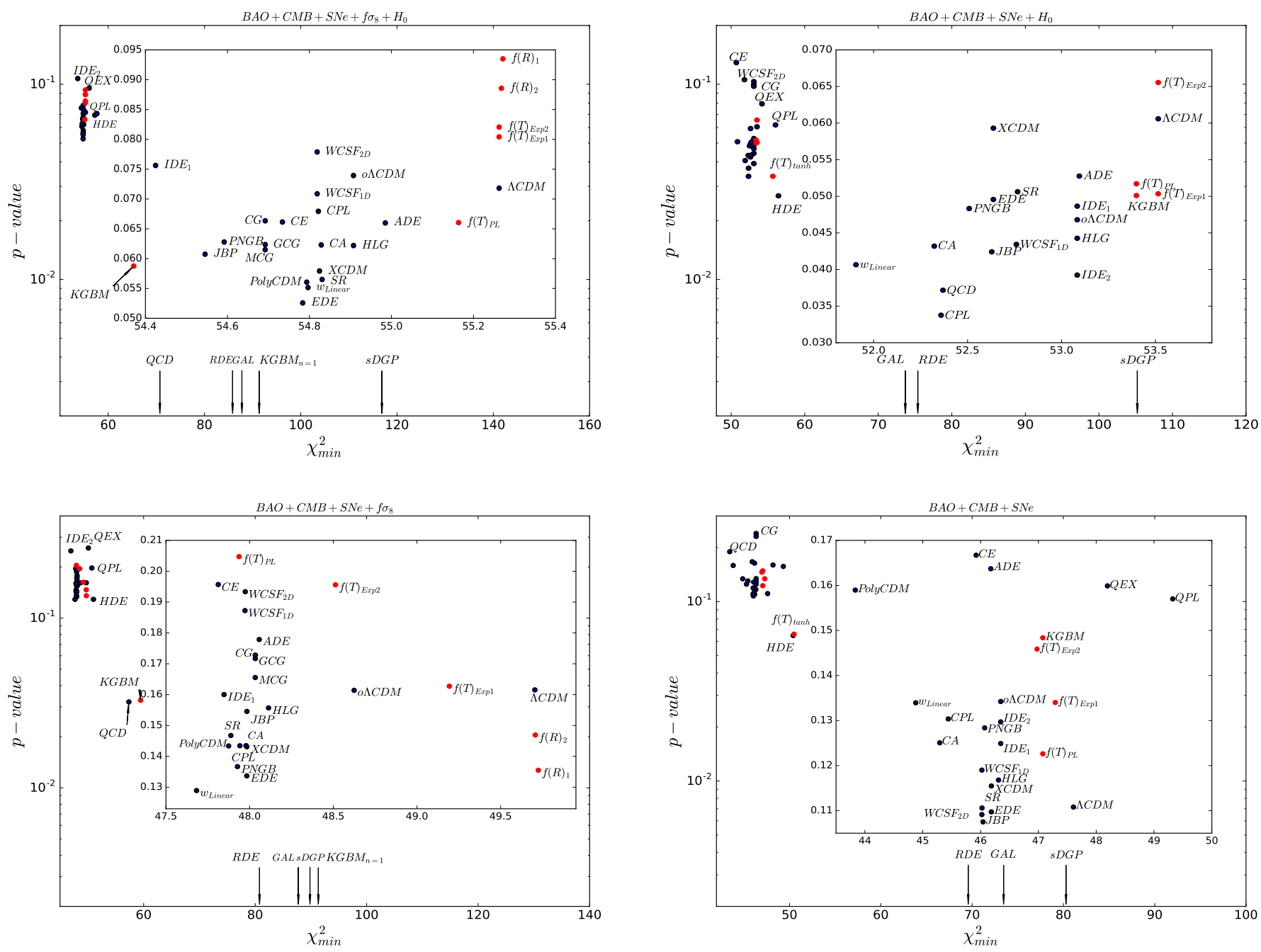

Figure 4. The $p$-value test for the dark energy (blue dots) and modified gravity (red dots) models by the use of the datasets: Top : $\mathrm{BAO}+\mathrm{CMB}+\mathrm{SNe}+f \sigma_{8}+\mathrm{H}_{0} ;$ Bottom : $\mathrm{BAO}+\mathrm{CMB}+\mathrm{SNe}+f \sigma_{8}$ The box inside the figure is the zoom-in region around the unidentified points. Note that the $f(T)_{\text {tanh }}$ model has a very high $\chi^{2}$ and a $p$-value that is effectively zero and does not appear here. The vertical arrows show the upper limit of the $p$-value for models which has none in the ensemble of the simulated data sets have a $\chi^{2}$ larger than the real data (Gehrels 1986).

The KGBM model survives with $p \sim 0.01$. The remaining models all imply that the data is a marginal outlier with $p \sim 0.05-0.11$. The data is most compatible with the IDE $_{2}$ model (though we remind the reader that this fact does not imply that this model is most probable, which is not something we can determine, at least using our methodology).

It is also well known that some tension exists between the LyaF BAO and Planck $\Lambda$ CDM model (Planck Collaboration et al. 2014, Aubourg et al. 2015). We investigate this problem for the models introduced here and the resulting $p$-value test is summarized in the last column of Table 1. We compare the $p$-value for the dataset $\mathrm{BAO}+\mathrm{CMB}$ with and without $\mathrm{LyaF} \mathrm{BAO}$. The addition of the LyaF BAO typically decreases the $p$-value by $0.1 \sim 0.3$, and indicates mild tension between the datasets. The difference of the $p$-values $\Delta p$ when a par-

Figure 5. The $p$-value test for the dark energy (blue dots) and modified gravity (red dots) models by the use of the datasets: Top : $\mathrm{BAO}+\mathrm{CMB}+\mathrm{SNe}+\mathrm{H}_{0} ;$ Bottom $: \mathrm{BAO}+\mathrm{CMB}+\mathrm{SNe}$.

ticular dataset is added can be used to characterize this tension. In the test of LyaF BAO, PolyCDM and QCD ghost models have a $p$-value difference smaller than 0.1 , which we believe indicates a mitigation of the tension, due to achieving a better $\chi^{2}$ than other models when the LyaF BAO is added (this is how we define "Yes" for these two models and "No" for all the other models in the last column of Table 1 . In the test of $\mathrm{H}_{0}$ tension, we note that adding $\mathrm{H}_{0}$ typically decreases the $p$-value by $0.05 \sim 0.1$. Although some models have $\Delta p$ smaller than 0.1 as a criterion for LyaF BAO test, none of these models performs much better than $\Lambda$ CDM, which implies that the tension of $\mathrm{H}_{0}$ still exists in these models and this is represented as "No" in Table 14. The result for linear growth data is obtained in a similar way.

We should emphasize here the point made in Wasserstein \& Lazar (2016), that whereas $p$-values can indicate how incompatible the data are with a specified statisti-

4 The Galileon model with tracker solution is ruled out by $p$ value, but the best-fit parameter indicates the $\mathrm{H}_{0}$ discrepancy does not exist, therefore we fill it with "Yes". 


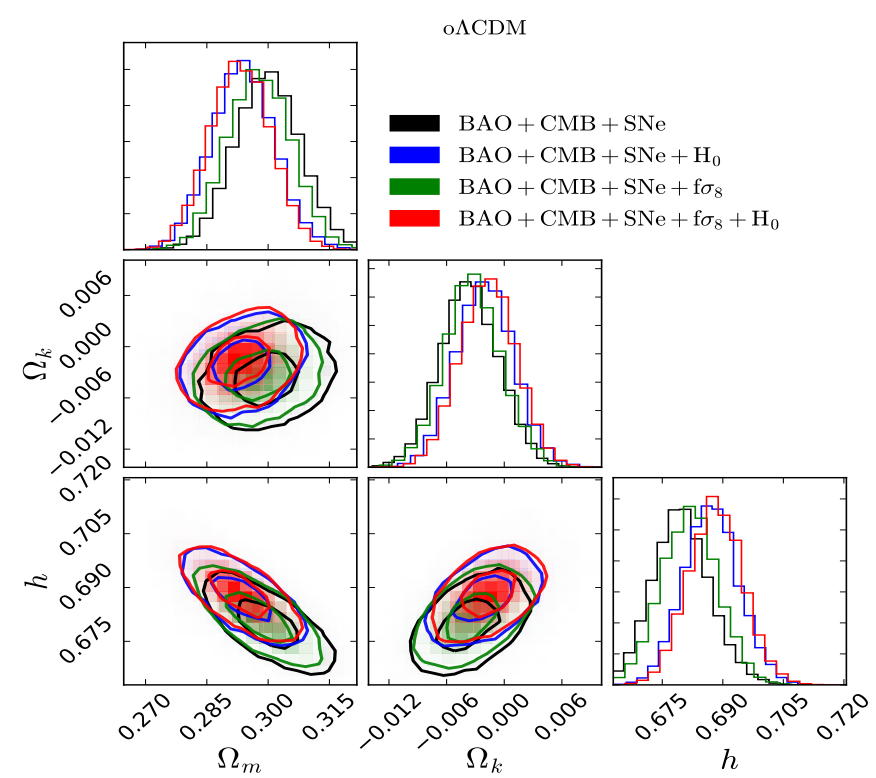

Figure 6. The $68.7 \%$ and $95.0 \%$ confidence regions of the parameters for the non-flat $\Lambda \mathrm{CDM}$. The diagonal panels show the one-dimensional probability distribution functions. The data combinations used are: $\mathrm{BAO}+\mathrm{CMB}+\mathrm{SNe}$ (black), $\mathrm{BAO}+\mathrm{CMB}+\mathrm{SNe}+\mathrm{H}_{0}$ (blue), $\mathrm{BAO}+\mathrm{CMB}+\mathrm{SNe}+f \sigma_{8} \quad$ (green), and $\mathrm{BAO}+\mathrm{CMB}+\mathrm{SNe}+f \sigma_{8}+\mathrm{H}_{0}$ (red). The color coding for the constraint results in the following sections is the same.

cal model, they do not measure the probability that the model is true, or the probability that the disagreement was produced by random chance alone. An extremely low $p$-value for a specific model does indicate an important incompatibility with the data, which can be reasonably used to rule out it as a successful theory (assuming that there is not some systematic issue affecting the data or its uncertainty estimates).

\section{ANALYSIS OF INDIVIDUAL MODELS}

We now turn to consider each of the cosmological models described in Section 2.1, both its goodness of fit and its cosmological constraints.

As we showed above, the simplest $\Lambda \mathrm{CDM}$ model explains the data roughly as well as any other model, at least vis-a-vis our chosen summary metric. Nevertheless it is interesting to explore what the constraints are on both the conventional cosmological parameters, and the additional parameters introduced in the more exotic models.

To examine these constraints, we use a Monte Carlo Markov Chain technique. Specifically, we use the python package emcee (Foreman-Mackey et al. 2013), which is based on an affine-invariant ensemble sampling algorithm (Goodman \& Weare 2010).

Figure 6 shows the confidence regions and onedimensional probability functions of the parameters for the non-flat $\Lambda \mathrm{CDM}$ model, under our four chosen parameter combinations. The impact of the $H_{0}$ data is evident from this figure; the best-fit value of $h$ increases by about $1 \sigma$ when the $\mathrm{H}_{0}$ measurement is added. The constraint on the spatial curvature $\Omega_{k}$ doesn't show strong deviation from flatness; $\Omega_{k}=0$ is well within the $1 \sigma$ region for all the data sets combined. Table 2 and 3 summarize the mean values and uncertainties of the parameters for the
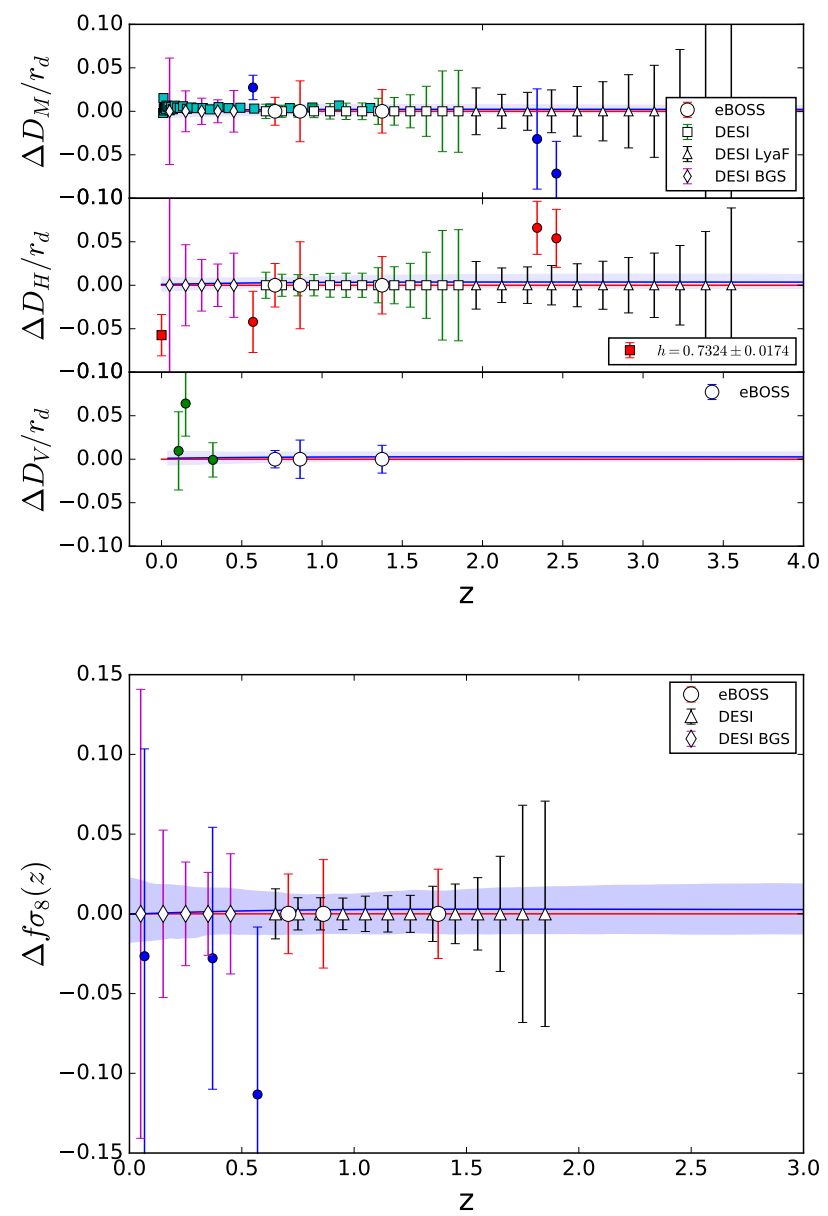

Figure 7. Predicted observables for non-flat $\Lambda$ CDM model based on current data. Top panel: the BAO distance scales; Bottom panel: $f \sigma_{8}$ from the linear perturbation theory. Quantities shown are the fraction differences from the flat $\Lambda$ CDM model. The horizontal red line at zero is the prediction from flat $\Lambda \mathrm{CDM}$ model. Filled symbols with error bars represent current observations: red circles are $\mathrm{BAO}$ measurements, cyan squares are $\mathrm{SNe}$, and the red square is $\mathrm{H}_{0}$. Open symbols with error bars are the forecasts for future experiments: circles are eBOSS (LRGs, ELGs, and QSOs), diamonds are the DESI Bright Galaxy Survey (DESI BGS), squares are the DESI LRG and ELG surveys, and triangles are the DESI Lyman $\alpha$ Forest survey (DESI LyaF). The blue line is the best-fit prediction from the non-flat $\Lambda \mathrm{CDM}$ model and the shaded region shows the $1 \sigma$ distribution given the MCMC constraints.

flat $\Lambda \mathrm{CDM}$ and non-flat $\Lambda \mathrm{CDM}$ model respectively. The $\mathrm{BIC}$ and AIC calculations are also shown. For models different than flat $\Lambda \mathrm{CDM}$ model, only the difference is shown in the following calculation.

Figure 7 shows the predicted observables under the non-flat $\Lambda \mathrm{CDM}$ models. These observables shown are the distance measures and $f \sigma_{8}$ across redshift. The predictions for these observables are shown as fractional deviations from the best-fit flat $\Lambda \mathrm{CDM}$ model. The band shown is the $1 \sigma$ variation of the observable under the MCMC sampling of the model parameters. We include current data and uncertainties as the filled symbols. Clearly in this model space the range of variation in observed quantities is quite small. In this figure, we also include projections for eBOSS (Dawson et al. 2016. Zhao et al. 2016) and DESI (Levi et al. 2013; DESI 2016) 


\begin{tabular}{llllll}
\hline \multicolumn{2}{c}{$\Lambda \mathrm{CDM}$} & & & \\
\hline Data & $\Omega_{m}$ & $h$ & $\sigma_{8}$ & $\mathrm{BIC}$ & $\mathrm{AIC}$ \\
\hline $\mathrm{BAO}+\mathrm{CMB}+\mathrm{SNe}$ & $0.301 \pm 0.008$ & $0.684 \pm 0.006$ & - & 58.9 & 53.6 \\
$\mathrm{BAO}+\mathrm{CMB}+\mathrm{SNe}+\mathrm{H}_{0}$ & $0.296 \pm 0.007$ & $0.689 \pm 0.006$ & - & 64.9 & 59.5 \\
$\mathrm{BAO}+\mathrm{CMB}+\mathrm{SNe}+f \sigma_{8}$ & $0.300 \pm 0.008$ & $0.685 \pm 0.006$ & $0.825 \pm 0.011$ & 65.0 & 57.7 \\
$\mathrm{BAO}+\mathrm{CMB}+\mathrm{SNe}+f \sigma_{8}+\mathrm{H}_{0}$ & $0.294 \pm 0.007$ & $0.690 \pm 0.006$ & $0.823 \pm 0.011$ & 70.7 & 63.3 \\
\hline
\end{tabular}

Table 2

Cosmological parameter constraints for the flat $\Lambda \mathrm{CDM}$ model, the values of the BIC and AIC are also shown in the last two columns.

\begin{tabular}{lllllll}
\hline \multicolumn{7}{c}{$o \Lambda \mathrm{CDM}$} \\
\hline Data & $\Omega_{m}$ & $\Omega_{k}$ & $h$ & $\sigma_{8}$ & $\Delta \mathrm{BIC}$ & $\Delta \mathrm{AIC}$ \\
\hline $\mathrm{BAO}+\mathrm{CMB}+\mathrm{SNe}$ & $0.300 \pm 0.008$ & $-0.003 \pm 0.003$ & $0.680 \pm 0.007$ & - & 2.5 & 0.7 \\
$\mathrm{BAO}+\mathrm{CMB}+\mathrm{SNe}+\mathrm{H}_{0}$ & $0.294 \pm 0.008$ & $-0.002 \pm 0.003$ & $0.687 \pm 0.007$ & - & 3.4 & 1.6 \\
$\mathrm{BAO}+\mathrm{CMB}+\mathrm{SNe}+f \sigma_{8}$ & $0.298 \pm 0.008$ & $-0.003 \pm 0.003$ & $0.682 \pm 0.007$ & $0.828 \pm 0.012$ & 2.7 & 0.9 \\
$\mathrm{BAO}+\mathrm{CMB}+\mathrm{SNe}+f \sigma_{8}+\mathrm{H}_{0}$ & $0.293 \pm 0.007$ & $-0.002 \pm 0.003$ & $0.688 \pm 0.007$ & $0.825 \pm 0.011$ & 3.5 & 1.6 \\
\hline
\end{tabular}

Table 3

Cosmological parameter constraints for the non-flat $\Lambda \mathrm{CDM}$ model. The last two columns show the difference of BIC and AIC compared with spatially flat $\Lambda \mathrm{CDM}$ model: $\Delta \mathrm{BIC}=\mathrm{BIC}_{o \Lambda \mathrm{CDM}}-\mathrm{BIC}_{\Lambda \mathrm{CDM}}, \Delta \mathrm{AIC}=\mathrm{AIC}_{o \Lambda \mathrm{CDM}}-\mathrm{AIC}_{\Lambda \mathrm{CDM}}$.

as open symbols.

4.1. Parameterization of equation of state $w(X C D M$, $C P L, J B P, w_{\text {Linear }}$ and $\left.P N G B\right)$

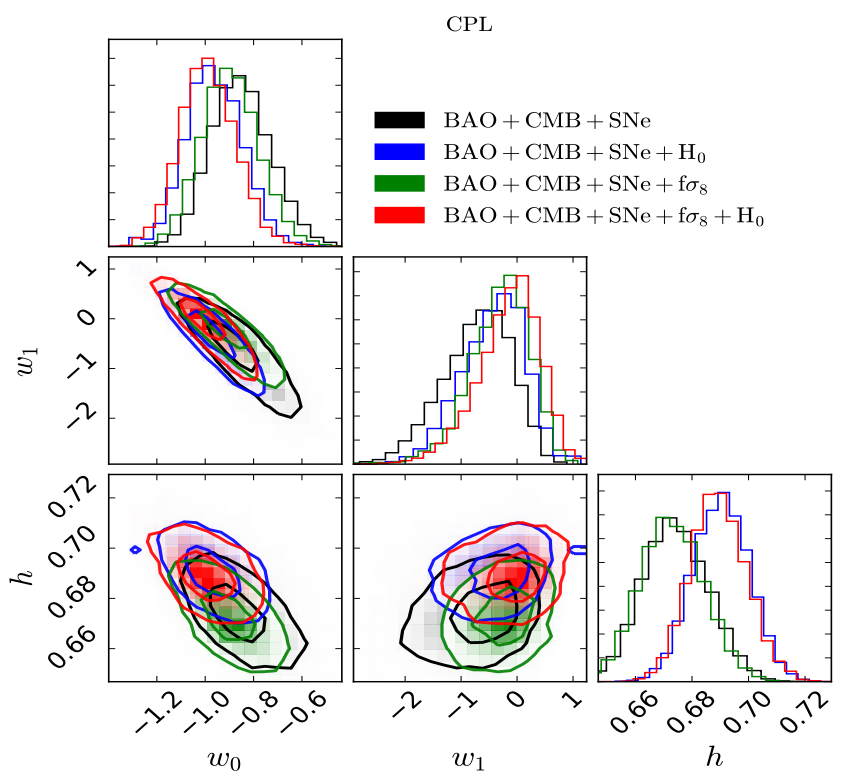

Figure 8. The $68.7 \%$ and $95.0 \%$ confidence regions of the parameters for non-flat CPL parameterization of $w$. The diagonal panels show the one-dimensional probability distribution functions.

Simple parameterizations of the equation of state of dark energy $w$ allow us to study aspects of the observed acceleration without explicit assumptions about its physical cause. As an example, Figure 8 presents the constraints on the CPL parameterization. A constant equation of state $\left(w_{1}=0\right)$ lies within the $95 \%$ distribution for all data combinations. The distribution of $h$ is strongly affected by the $H_{0}$ data. The other parametrizations (XCDM, JBP, and $w_{\text {Linear }}$ ) behave very similarly.

The Pseudo-Nambu Goldstone Boson (PNGB) model has a well motivated physical background, but the cosmological solution is represented by a parameterization of $w$, which is a perturbation around -1 . In this model, we find similar results as the CPL model. The constraints on the parameters are displayed in Figure 9. The parameter $F$ is restricted to a limited range due to the numerical considerations, but it is clear that the upper bound is much larger than illustrated. The constraint on $w_{0}$ is consistent with -1 .

Tables 4 to 8 summarize the constraints on the XCDM, CPL, JBP, Linear parameterization, and PNGB models. The values of $w_{0}$ from different datasets prefer a quintessence-like result to a phantom-like result in the very late universe. The result doesn't show clear evidence of the dynamical evolution of dark energy. The well-known tension of $H_{0}$ is a little larger than $1 \sigma$.

The $p$-value test of these models all show similar behavior. The addition of the local measurement of $\mathrm{H}_{0}$ degrades the $p$-value to around $p \sim 0.05$. Compared with the standard $\Lambda$ CDM model, their flexibility allows them to lower $\chi^{2}$ by about 1 to 2 , but not a much better $p$-value.

Figure 10 shows the predicted observables under the CPL model, which are illustrative of most of these parametrizations aside from PNGB. Whereas PNGB behaves similarly to $\Lambda \mathrm{CDM}$, for the others the predicted observations have a broader range, especially in $f \sigma_{8}$. The fractional variations do not appear preferentially high at any range of redshifts.

\subsection{Casimir effect (CE)}

For the CE model, the constraint on the parameter $\Omega_{\mathrm{c}}$ together with the spatial curvature $\Omega_{k}$ (marginalizing the other parameters in the Casimir-effect model) is presented in Figure 11 and Table 9. A flat FRW cosmology is within the than $2 \sigma$ contours for all four data combinations. The geometrical probes favor a larger deviation of $\Omega_{k}$ from 0 ; this deviation is suppressed when including the linear growth data.

The upper bound of the contribution from the negative radiation-like term is much smaller than $1.0 \%$ at a $95 \%$ confidence level. The results for $h$ show that despite the improved $p$-value, the disagreement between the large scale structure data and the local measurement of $\mathrm{H}_{0}$ remains.

In the $p$-value test, for the geometrical probes $\mathrm{CE}$ gives better $p$-value than the $\Lambda \mathrm{CDM}$ model, and in fact 


\begin{tabular}{|c|c|c|c|c|c|c|c|}
\hline \multicolumn{8}{|c|}{ XCDM } \\
\hline Data & $\Omega_{m}$ & $\Omega_{k}$ & $w$ & $h$ & $\sigma_{8}$ & $\Delta \mathrm{BIC}$ & $\Delta \mathrm{AIC}$ \\
\hline $\mathrm{BAO}+\mathrm{CMB}+\mathrm{SNe}$ & $0.302 \pm 0.010$ & $-0.003 \pm 0.003$ & $-0.98 \pm 0.05$ & $0.677 \pm 0.011$ & - & 6.1 & 2.6 \\
\hline $\mathrm{BAO}+\mathrm{CMB}+\mathrm{SNe}+\mathrm{H}_{0}$ & $0.292 \pm 0.009$ & $-0.003 \pm 0.003$ & $-1.03 \pm 0.05$ & $0.690 \pm 0.010$ & - & 6.7 & 3.1 \\
\hline $\mathrm{BAO}+\mathrm{CMB}+\mathrm{SNe}+f \sigma_{8}$ & $0.303 \pm 0.010$ & $-0.002 \pm 0.003$ & $-0.96 \pm 0.05$ & $0.675 \pm 0.011$ & $0.822 \pm 0.013$ & 5.9 & 2.3 \\
\hline $\mathrm{BAO}+\mathrm{CMB}+\mathrm{SNe}+f \sigma_{8}+\mathrm{H}_{0}$ & $0.292 \pm 0.009$ & $-0.002 \pm 0.003$ & $-1.01 \pm 0.05$ & $0.690 \pm 0.010$ & $0.826 \pm 0.014$ & 7.3 & 3.6 \\
\hline
\end{tabular}

Table 4

Cosmological parameter constraints for the XCDM model.

\begin{tabular}{|c|c|c|c|c|c|c|c|c|}
\hline \multicolumn{9}{|c|}{ CPL parameterization } \\
\hline Data & $\Omega_{m}$ & $\Omega_{k}$ & $w_{0}$ & $w_{1}$ & $h$ & $\sigma_{8}$ & $\Delta \mathrm{BIC}$ & $\Delta \mathrm{AIC}$ \\
\hline $\mathrm{BAO}+\mathrm{CMB}+\mathrm{SNe}$ & $0.305 \pm 0.011$ & $-0.006 \pm 0.004$ & $-0.86 \pm 0.12$ & $-0.7 \pm 0.7$ & $0.674 \pm 0.012$ & - & 9.1 & 3.8 \\
\hline $\mathrm{BAO}+\mathrm{CMB}+\mathrm{SNe}+\mathrm{H}_{0}$ & $0.293 \pm 0.009$ & $-0.004 \pm 0.004$ & $-0.97 \pm 0.12$ & $-0.4 \pm 0.5$ & $0.690 \pm 0.010$ & - & 10.2 & 4.8 \\
\hline $\mathrm{BAO}+\mathrm{CMB}+\mathrm{SNe}+f \sigma_{8}$ & $0.305 \pm 0.011$ & $-0.004 \pm 0.004$ & $-0.90 \pm 0.12$ & $-0.3 \pm 0.5$ & $0.673 \pm 0.011$ & $0.829 \pm 0.019$ & 9.7 & 4.2 \\
\hline $\mathrm{BAO}+\mathrm{CMB}+\mathrm{SNe}+f \sigma_{8}+\mathrm{H}_{0}$ & $0.293 \pm 0.009$ & $-0.002 \pm 0.004$ & $-0.99 \pm 0.11$ & $-0.1 \pm 0.5$ & $0.690 \pm 0.010$ & $0.829 \pm 0.020$ & 11.1 & 5.6 \\
\hline
\end{tabular}

Table 5

Cosmological parameter constraints for the CPL parameterization of the equation of state.

\begin{tabular}{llllll}
\hline \multicolumn{7}{c}{ JBP parameterization } & & & \\
\hline Data & $w_{0}$ & $w_{1}$ & $h$ & $\Delta$ BIC & $\Delta$ AIC \\
\hline $\mathrm{BAO}+\mathrm{CMB}+\mathrm{SNe}$ & $-0.90 \pm 0.18$ & $-0.6 \pm 1.2$ & $0.674 \pm 0.011$ & 9.7 & 4.4 \\
$\mathrm{BAO}+\mathrm{CMB}+\mathrm{SNe}+\mathrm{H}_{0}$ & $-1.01 \pm 0.16$ & $-0.2 \pm 1.2$ & $0.690 \pm 0.010$ & 10.5 & 5.1 \\
$\mathrm{BAO}+\mathrm{CMB}+\mathrm{SNe}+f \sigma_{8}$ & $-0.94 \pm 0.16$ & $-0.1 \pm 1.1$ & $0.675 \pm 0.011$ & 9.8 & 4.3 \\
$\mathrm{BAO}+\mathrm{CMB}+\mathrm{SNe}+f \sigma_{8}+\mathrm{H}_{0}$ & $-1.08 \pm 0.16$ & $0.5 \pm 1.1$ & $0.692 \pm 0.010$ & 10.8 & 5.3 \\
\hline
\end{tabular}

Table 6

Cosmological constraints for a selection of parameters for the JBP parameterization of the equation of state.

\begin{tabular}{llllll}
\hline \multicolumn{7}{l}{ Linear parameterization } & & \\
\hline Data & $w_{0}$ & $w_{1}$ & $h$ & $\Delta$ BIC & $\Delta$ AIC \\
\hline BAO+CMB+SNe & $-0.86 \pm 0.09$ & $-0.4 \pm 0.3$ & $0.673 \pm 0.010$ & 8.6 & 3.3 \\
$\mathrm{BAO}+\mathrm{CMB}+\mathrm{SNe}+\mathrm{H}_{0}$ & $-0.93 \pm 0.09$ & $-0.4 \pm 0.4$ & $0.689 \pm 0.009$ & 9.7 & 4.4 \\
$\mathrm{BAO}+\mathrm{CMB}+\mathrm{SNe}+f \sigma_{8}$ & $-0.87 \pm 0.08$ & $-0.3 \pm 0.3$ & $0.672 \pm 0.011$ & 9.5 & 4.0 \\
$\mathrm{BAO}+\mathrm{CMB}+\mathrm{SNe}+f \sigma_{8}+\mathrm{H}_{0}$ & $-0.95 \pm 0.07$ & $0.2 \pm 0.2$ & $0.689 \pm 0.010$ & 11.1 & 5.5 \\
\hline
\end{tabular}

Table 7

Cosmological constraints for a selection of parameters for the Linear parameterization of the equation of state.

\begin{tabular}{|c|c|c|c|c|}
\hline \multicolumn{5}{|c|}{ PNGB } \\
\hline Data & $w_{0}$ & $F$ & $\Delta \mathrm{BIC}$ & $\Delta \mathrm{AIC}$ \\
\hline $\mathrm{BAO}+\mathrm{CMB}+\mathrm{SNe}$ & $-0.94 \pm 0.11$ & $4.85_{-2.79}$ & 9.7 & 4.5 \\
\hline $\mathrm{BAO}+\mathrm{CMB}+\mathrm{SNe}+\mathrm{H}_{0}$ & $-1.06 \pm 0.10$ & $5.06_{-2.97}$ & 10.3 & 5.0 \\
\hline $\mathrm{BAO}+\mathrm{CMB}+\mathrm{SNe}+f \sigma_{8}$ & $-0.92 \pm 0.10$ & $4.79-3.15$ & 9.7 & 4.2 \\
\hline $\mathrm{BAO}+\mathrm{CMB}+\mathrm{SNe}+f \sigma_{8}+\mathrm{H}_{0}$ & $-1.04 \pm 0.11$ & $5.24_{-2.83}$ & 10.9 & 5.3 \\
\hline
\end{tabular}

Table 8

Cosmological constraints for a selection of parameters for the PNGB model.

is the model under which the data is least discrepant when $\mathrm{H}_{0}$ is included. However, adding linear growth data suppresses the ability of CE to introduce curvature and reduces its advantage over other models.

When we consider the predicted observables under this model (the analog of Figure 7) we find it is very similar to that for non-flat $\Lambda \mathrm{CDM}$ - there is not much more freedom allowed by this model regarding the observables considered here.

\subsection{Cardassian expansion ( $C A$ )}

The Cardassian expansion tested here (specifically the version introduced by Wang et al. 2003) is a direct modification of the Friedmann equation Eq.(15). However, the model also can be thought of as a generalization of the XCDM model. A number of previous studies have considered constraints on this model (Zhu \& Fujimoto 2002, 2003, Sen \& Sen 2003, Zhu et al. 2004 Frith 2004. Alcaniz et al. 2005; Xu 2012).

The constraints on the parameters in this paper are shown in Figure 12 and Table 10 . The deviation from $\Lambda$ CDM model with $q=1$ and $n=0$ is not significant. The degeneracy between $q$ and $n$ is clear in various data combinations; generally a broad range of each is allowed as long as the value for the other is such that the expansion and growth rate remain similar to $\Lambda$ CDM.

This property also appears in the $p$-value test. The tiny allowed deviation from the $\Lambda$ CDM model means that it behaves similarly in terms of compatibility with the data.

This model behaves similarly to parametrizations such as CPL in terms of its predicted observables given current 


\begin{tabular}{|c|c|c|c|c|}
\hline \multicolumn{5}{|c|}{ Casimir effect } \\
\hline Data & $\Omega_{k}$ & $\Omega_{\text {Cas }}$ & $\Delta \mathrm{BIC}$ & $\Delta \mathrm{AIC}$ \\
\hline $\mathrm{BAO}+\mathrm{CMB}+\mathrm{SNe}$ & $-0.016_{-0.036}^{+0.009}$ & $0.0_{-0.001}$ & 5.8 & 2.3 \\
\hline $\mathrm{BAO}+\mathrm{CMB}+\mathrm{SNe}+\mathrm{H}_{0}$ & $-0.050_{-0.012}^{+0.040}$ & $-0.001-0.002$ & 4.8 & 1.2 \\
\hline $\mathrm{BAO}+\mathrm{CMB}+\mathrm{SNe}+f \sigma_{8}$ & $-0.006 \pm 0.007$ & $0.0_{-0.0002}$ & 5.8 & 2.1 \\
\hline $\mathrm{BAO}+\mathrm{CMB}+\mathrm{SNe}+f \sigma_{8}+\mathrm{H}_{0}$ & $-0.007_{-0.008}^{+0.007}$ & $0.0_{-0.0001}$ & 7.2 & 3.5 \\
\hline
\end{tabular}

Table 9

Cosmological constraints for a selection of parameters for the Casimir effect.

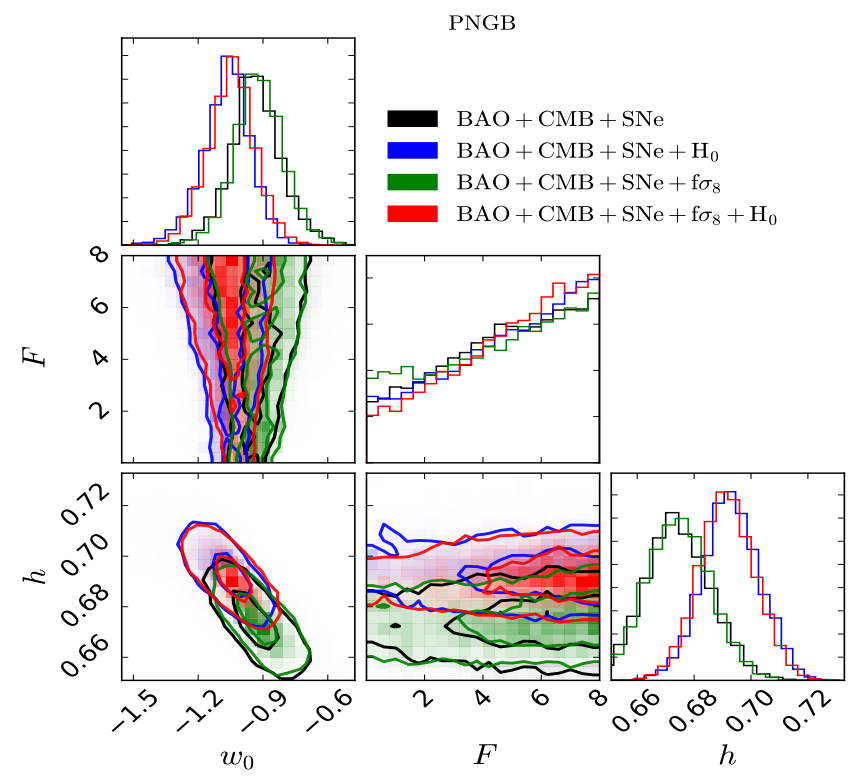

Figure 9. The $68.7 \%$ and $95.0 \%$ confidence regions of the parameters $w_{0}, F$ and $h$ for non-flat PNGB model. The diagonal panels show the one-dimensional probability distribution functions.

data (the results look similar to Figure 10 .

\subsection{Early dark energy (EDE)}

Figure 13 and Table 11 present constraints on the early dark energy model from the data. The dark energy component has effects on early times and leaves its footprint on the matter perturbation. However, the data constraint suggests a small energy fraction, with $\Omega_{\text {de }}^{e}$ less than 0.07 at $95 \%$ confidence (in agreement with Doran \& Robbers 2006). Explaining the linear growth data does not require this early energy, though all the measurements are compatible with some modest amount of it. The different data combinations give consistent results regarding $\Omega_{d e}^{e}$. As emphasized in Aubourg et al. (2015); Shi \& Baugh (2016), a detailed analysis of the CMB power spectrum (not limited to the "compressed" CMB data used here) can impose much tighter constraints on early dark energy, at least for this specific model.

The nearly-negligible contribution of early dark energy doesn't improve the fit to the data significantly, therefore the limited improvement of $\chi^{2}$ is not able to offset the added model complexity, and the $p$-value for this model is comparable to that for the $\Lambda \mathrm{CDM}$ model.

Early dark energy models constrained by current data have predicted observables with a range similar to that shown in Figure 10 for the CPL parametrization.

\subsection{Slow roll dark energy (SR)}
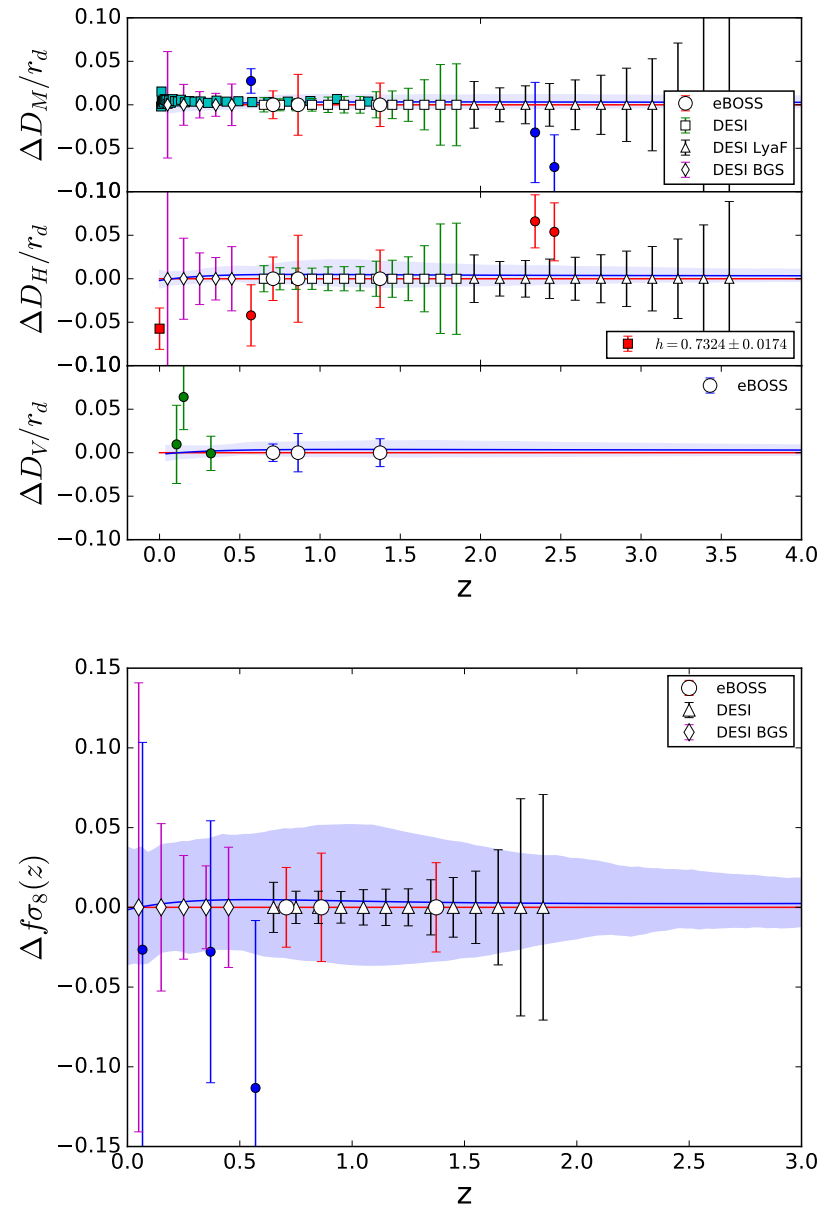

Figure 10. Similar to Figure 7 for the CPL parametrization.

The slow roll dark energy scenario has the same number of parameters as the XCDM model and is very similar in form, especially for small deviations from $w=-1$. The constraints are shown in Figure 14, which yields $\delta w_{0}=0.01 \pm 0.06$ for all datasets combined, and no significant departure from zero for any combination of data. Table 12 presents the mean and uncertainties of the parameters for the slow roll dark energy model.

The $p$-value for this model is quite similar to the early dark energy model, as their deviation from the $\Lambda \mathrm{CDM}$ model is constrained to be small. The predicted observables given current data are even tighter, very similar to that found for non-flat $\Lambda$ CDM in Figure 7 .

\subsection{Parameterization of the Hubble parameter}

(PolyCDM, HLG) 


\begin{tabular}{lllll}
\hline \multicolumn{5}{c}{ Cardassian expansion } \\
\hline Data & $q$ & $n$ & $\Delta \mathrm{BIC}$ & $\Delta \mathrm{AIC}$ \\
\hline $\mathrm{BAO}+\mathrm{CMB}+\mathrm{SNe}$ & $2.3_{-1.0}^{+2.7}$ & $0.36_{-0.20}^{+0.10}$ & 9.0 & 3.7 \\
$\mathrm{BAO}+\mathrm{CMB}+\mathrm{SNe}+\mathrm{H}_{0}$ & $1.6_{-0.8}^{+1.3}$ & $0.21_{-0.35}^{+0.16}$ & 10.2 & 4.8 \\
$\mathrm{BAO}+\mathrm{CMB}+\mathrm{SNe}+f \sigma_{8}$ & $1.3_{-0.5}^{+0.9}$ & $0.19_{-0.34}^{+0.18}$ & 9.8 & 4.3 \\
$\mathrm{BAO}+\mathrm{CMB}+\mathrm{SNe}+f \sigma_{8}+\mathrm{H}_{0}$ & $1.1_{-0.4}^{+0.8}$ & $0.02_{-0.41}^{+0.26}$ & 11.1 & 5.6 \\
\hline
\end{tabular}

Table 10

Cosmological constraints for a selection of parameters for the Cardassian expansion model.

\begin{tabular}{lllll}
\hline \multicolumn{5}{c}{ Early dark energy } \\
\hline Data & $\Omega_{d}$ & $w_{0}$ & $\Delta \mathrm{BIC}$ & $\Delta \mathrm{AIC}$ \\
\hline $\mathrm{BAO}+\mathrm{CMB}+\mathrm{SNe}$ & $0.0^{+0.025}$ & $-1.01_{-0.07}^{+0.06}$ & 9.9 & 4.6 \\
$\mathrm{BAO}+\mathrm{CMB}+\mathrm{SNe}+\mathrm{H}_{0}$ & $0.0^{+0.029}$ & $-1.06_{-0.07}^{+0.06}$ & 10.5 & 5.1 \\
$\mathrm{BAO}+\mathrm{CMB}+\mathrm{SNe}+f \sigma_{8}$ & $0.0^{+0.023}$ & $-1.00 \pm 0.06$ & 9.8 & 4.3 \\
$\mathrm{BAO}+\mathrm{CMB}+\mathrm{SNe}+f \sigma_{8}+\mathrm{H}_{0}$ & $0.0^{+0.025}$ & $-1.06_{-0.07}^{+0.06}$ & 11.1 & 5.5 \\
\hline
\end{tabular}

Table 11

Cosmological constraints for a selection of parameters for the Early dark energy model.

\begin{tabular}{lllll}
\hline \multicolumn{5}{c}{ Slow roll dark energy } \\
\hline Data & $\Omega_{m}$ & $\delta w_{0}$ & $\Delta$ BIC & $\Delta$ AIC \\
\hline BAO+CMB+SNe & $0.304 \pm 0.011$ & $0.04 \pm 0.07$ & 5.9 & 2.4 \\
$\mathrm{BAO}+\mathrm{CMB}+\mathrm{SNe}+\mathrm{H}_{0}$ & $0.291 \pm 0.009$ & $-0.04 \pm 0.07$ & 6.8 & 3.3 \\
$\mathrm{BAO}+\mathrm{CMB}+\mathrm{SNe}+f \sigma_{8}$ & $0.304 \pm 0.010$ & $0.07 \pm 0.07$ & 5.8 & 2.2 \\
$\mathrm{BAO}+\mathrm{CMB}+\mathrm{SNe}+f \sigma_{8}+\mathrm{H}_{0}$ & $0.292 \pm 0.009$ & $-0.01 \pm 0.06$ & 7.3 & 3.6 \\
\hline
\end{tabular}

Table 12

Cosmological constraints for a selection of parameters for the Slow roll dark energy model.

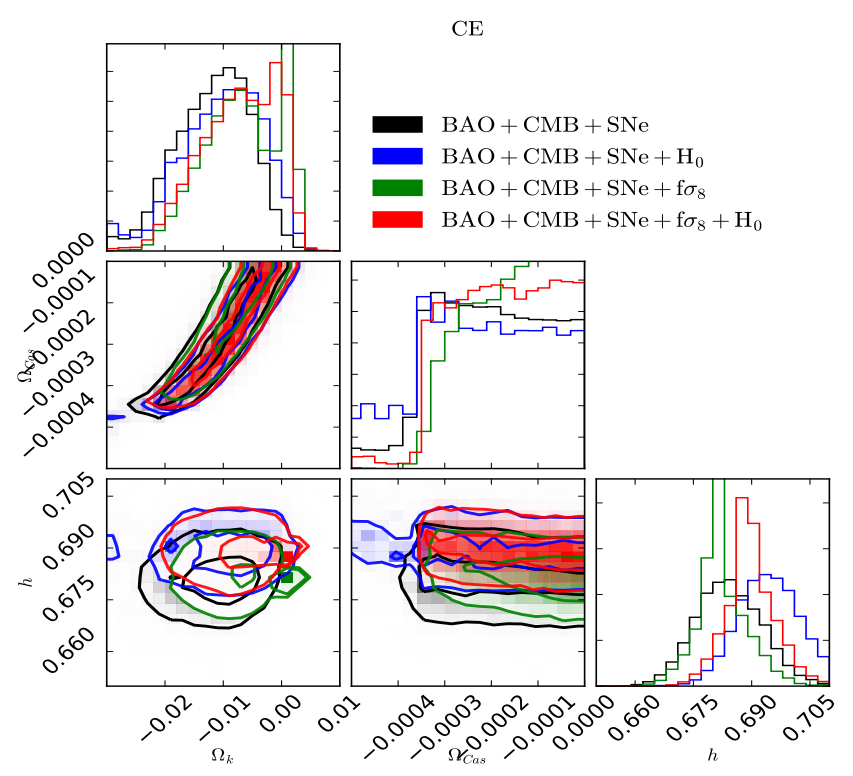

Figure 11. The $68.7 \%$ and $95.0 \%$ confidence regions of the parameters for the Casimir-effect cosmology.

Figure 15 presents the constraints on the parameters of two phenomenological parameterizations of the Hubble parameter: the PolyCDM model (Eq. 22) and the HLG model (Eq. 23). The figure shows clear differences in best fit parameters depending on the datasets used for the PolyCDM model; because the model is quite flexible, it results in quite different parameters if some data is excluded (though none that are more than $2 \sigma$ away from $\Lambda \mathrm{CDM})$. As the measurements of Hubble constant and

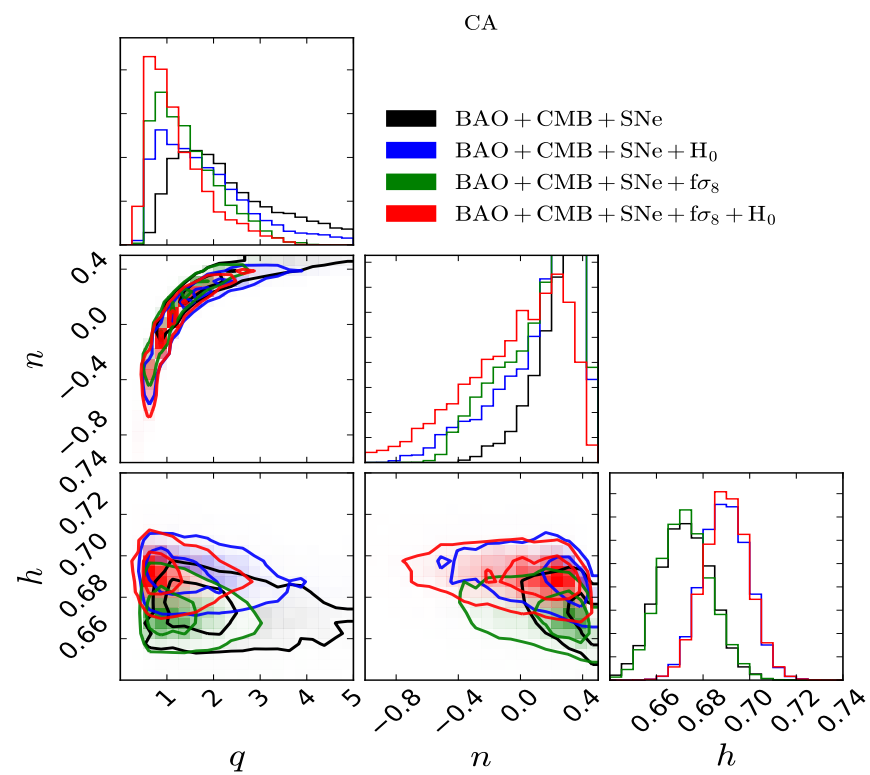

Figure 12. The $68.7 \%$ and $95.0 \%$ confidence regions of the parameters for the Cardassian expansion. The diagonal panels show the one-dimensional probability distribution functions.

linear growth are added, the parameters $\Omega_{m 1}$ and $\Omega_{m 2}$ approach 0.0 , which recovers the standard $\Lambda$ CDM model. In other words, a non-zero value of these two parameters can fit certain datasets better than $\Lambda$ CDM, but there is no such choice when all the datasets are included. The same effect is found in the logarithmic model. For different data samples, the $\Lambda$ CDM model is well within $1 \sigma$ confidence region. Inclusion of $\mathrm{H}_{0}$ pulls $h$ to higher values but does not resolve the tension of $\mathrm{H}_{0}$ with the other 


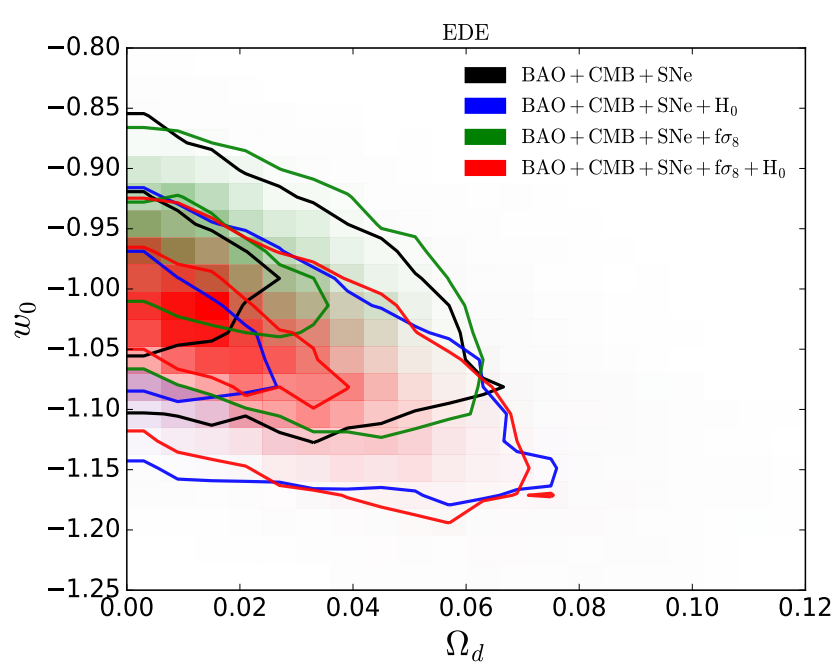

Figure 13. The $68.7 \%$ and $95.0 \%$ confidence regions of the parameters $\Omega_{d e}^{e}$ and $w_{0}$ for non-flat early dark energy model.

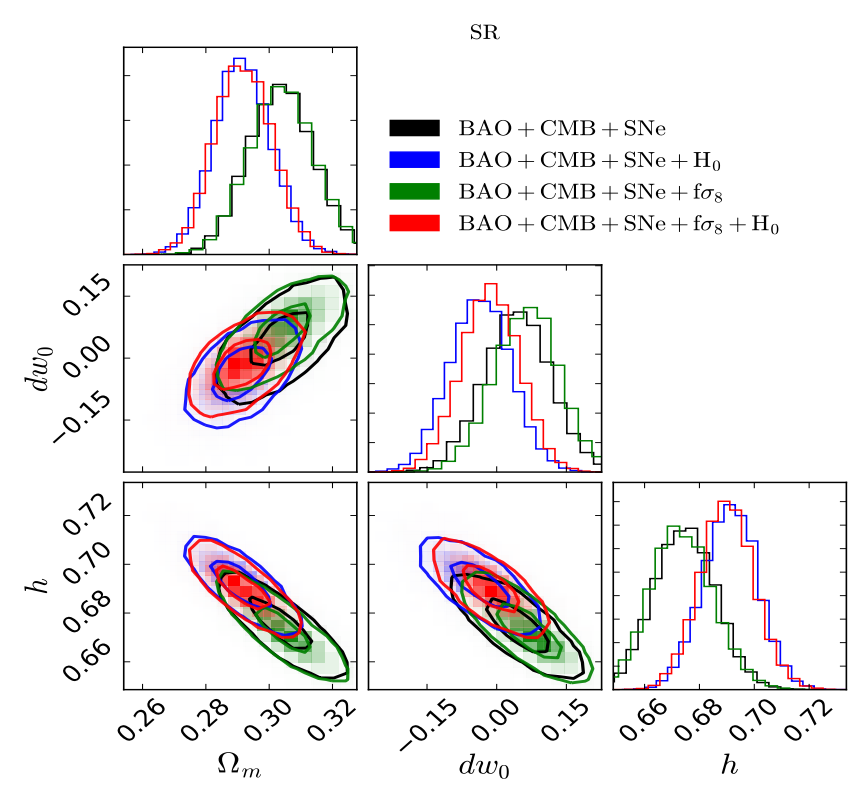

Figure 14. The $68.7 \%$ and $95.0 \%$ confidence regions of the parameters $\Omega_{m}$ and $d w_{0}$ for non-flat slow roll dark energy model. The diagonal panels show the one-dimensional probability distribution functions.

data. Table 13 and 14 summarize the constraint results for these two models respectively.

The $p$-value test of these two models reveal similar patterns. For instance, when the local measurement of $\mathrm{H}_{0}$ and linear growth data are excluded, the PolyCDM model gives a better $p$-value than $\Lambda \mathrm{CDM}$ model. As more data are added, PolyCDM is constrained to be closer to $\Lambda \mathrm{CDM}$ and this difference is reduced.

Because of the increased freedom in PolyCDM, the predicted observables are somewhat broader than other generalizations like CPL. Figure 16 shows these predicted observables. On the other hand, HLG has predicted observables similar to those for $\Lambda \mathrm{CDM}$ (Figure 7).
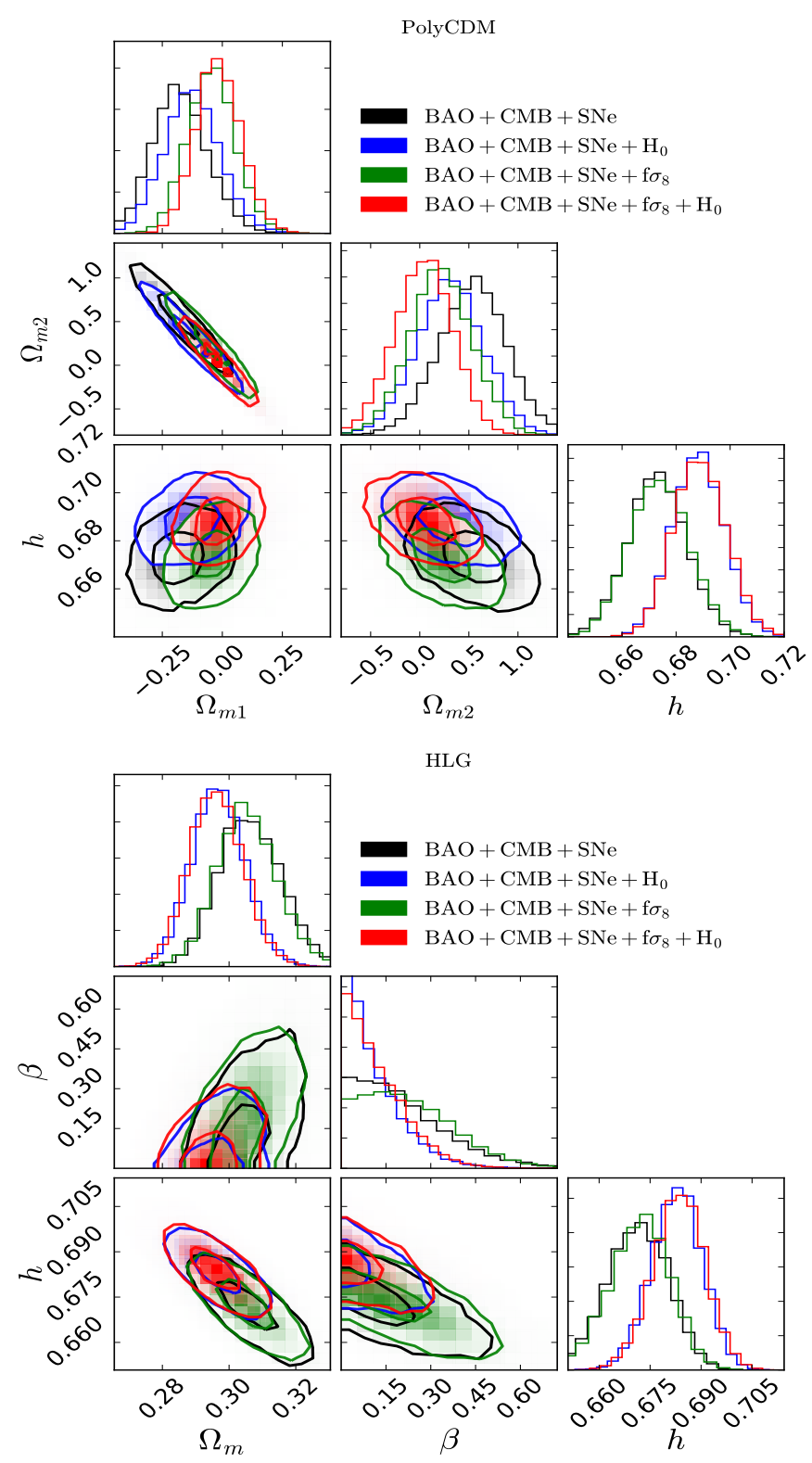

Figure 15. The $68.7 \%$ and $95.0 \%$ confidence regions of the parameters for two parameterizations of the Hubble parameter: the PolyCDM model (Top panel) and the Logarithmic model (Bottom panel). The diagonal panels show the one-dimensional probability distribution functions.

\subsection{Chaplygin gas (CG, GCG, $M C G)$}

The Chaplygin gas model has several generalizations that can produce cosmic acceleration. We present constraints on the Generalized Chaplygin gas model (GCG) parameters in Figure 17, but the results for the other Chaplygin gas models are similar. This model does not show significant deviations from the $\Lambda$ CDM model. The allowed deviation from a cosmological constant is small and the $\Lambda$ CDM model is well within the $1 \sigma$ confidence regions. Table 15, 16 and 17 summarize the constraints on the parameters for these Chaplygin gas models. For recent discussions about the cosmological constraints of this model see Park et al. (2010), Paul \& Thakur (2013), Wang et al. (2013), Lu et al. (2015), Sharov (2016), and 


\begin{tabular}{lllll}
\hline \multicolumn{5}{c}{ PolyCDM model } \\
\hline Data & $\Omega_{m 1}$ & $\Omega_{m 2}$ & $\Delta$ BIC & $\Delta$ AIC \\
\hline $\mathrm{BAO}+\mathrm{CMB}+\mathrm{SNe}$ & $-0.19_{-0.11}^{+0.12}$ & $0.55_{-0.35}^{+0.33}$ & 7.5 & 2.2 \\
$\mathrm{BAO}+\mathrm{CMB}+\mathrm{SNe}+\mathrm{H}_{0}$ & $-0.13_{-0.11}^{+0.12}$ & $0.31 \pm 0.34$ & 8.7 & 3.3 \\
$\mathrm{BAO}+\mathrm{CMB}+\mathrm{SNe}+f \sigma_{8}$ & $-0.05 \pm 0.10$ & $0.21_{-0.31}^{+0.32}$ & 9.7 & 4.2 \\
$\mathrm{BAO}+\mathrm{CMB}+\mathrm{SNe}+f \sigma_{8}+\mathrm{H}_{0}$ & $-0.02_{-0.09}^{+0.10}$ & $0.04 \pm 0.30$ & 11.1 & 5.5 \\
\hline
\end{tabular}

Table 13

Cosmological constraints for a selection of parameters for the PolyCDM dark energy model.

\begin{tabular}{lllll}
\hline \multicolumn{5}{c}{ Logarithmic parameterization } \\
\hline Data & $\Omega_{m}$ & $\beta$ & $\Delta \mathrm{BIC}$ & $\Delta \mathrm{AIC}$ \\
\hline $\mathrm{BAO}+\mathrm{CMB}+\mathrm{SNe}$ & $0.306 \pm 0.009$ & $0.04^{+0.18}$ & 6.2 & 2.7 \\
$\mathrm{BAO}+\mathrm{CMB}+\mathrm{SNe}+\mathrm{H}_{0}$ & $0.296 \pm 0.008$ & $0.00^{+0.12}$ & 7.1 & 3.6 \\
$\mathrm{BAO}+\mathrm{CMB}+\mathrm{SNe}+f \sigma_{8}$ & $0.305 \pm 0.009$ & $0.13^{+0.17}$ & 6.1 & 2.4 \\
$\mathrm{BAO}+\mathrm{CMB}+\mathrm{SNe}+f \sigma_{8}+\mathrm{H}_{0}$ & $0.296 \pm 0.008$ & $0.00^{+0.12}$ & 7.3 & 3.6 \\
\hline
\end{tabular}

Table 14

Cosmological constraints for a selection of parameters for the logarithmic parameterization of the Friedmann equation.
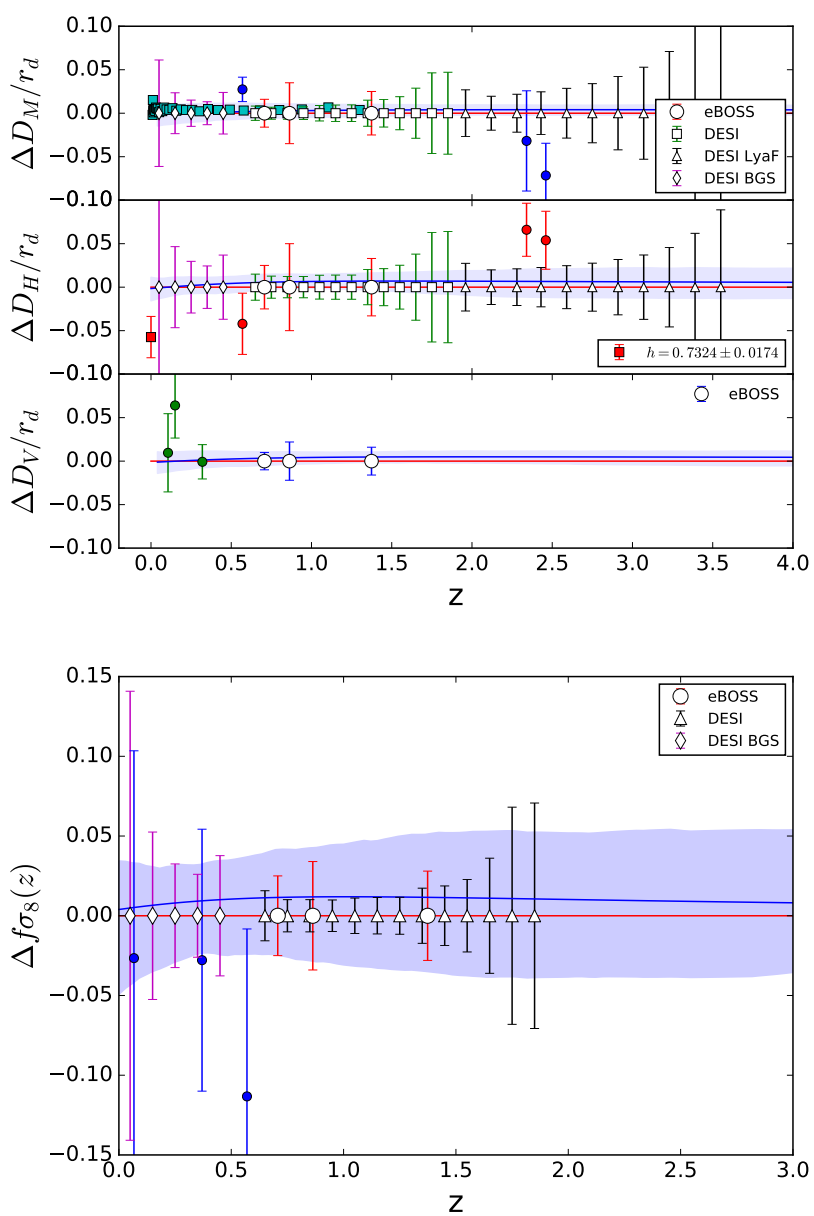

Figure 16. Similar to Figure 7 for the PolyCDM model.

references therein.

The Chaplygin gas models give the most favorable $p$ values relative to other models when only the geometrical data are included. Adding either $\mathrm{H}_{0}$ data or linear growth data makes the $p$-value less favorable and more comparable to other models.

The predicted observables for this model are similar to that for CPL (Figure 10). As expected, the GCG and MCG models show somewhat more variation in their predictions.

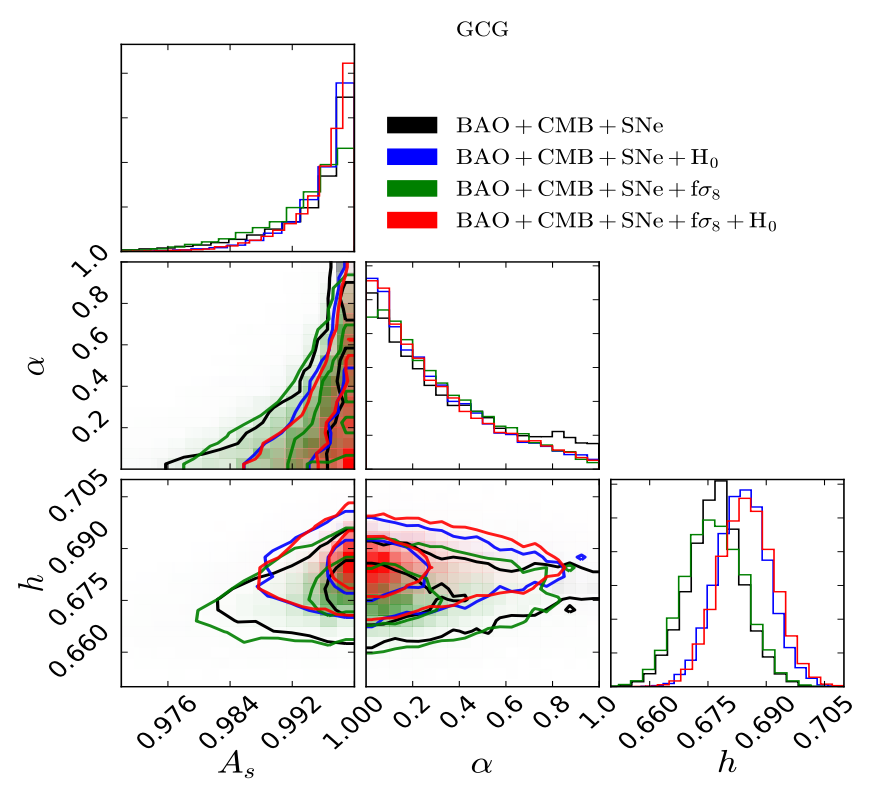

Figure 17. The $68.7 \%$ and $95.0 \%$ confidence regions of the parameters for the Generalized Chaplygin gas model. The diagonal panels show the one-dimensional probability distribution functions.

\subsection{Interacting $D E$ and $D M\left(I D E_{1}, I D E_{2}, I D E_{3}\right)$}

Figure 18 shows the constraints on the coupled dark energy models. This model mimics the expansion of the $\Lambda \mathrm{CDM}$ model. The constraint on the coupling between $\mathrm{DE}$ and DM is through the linear growth data. We here consider two parameterizations of the equation of state of the coupled dark energy. Compared with Fay (2016), which uses linear growth data only, the combination with the geometrical probes provide tighter constraints, as expected due to the better constraint on the dark matter component. Similarly, the degeneracy between the parameters is also consistent with the result using growth data only. The constraints on this model are shown in 


\begin{tabular}{|c|c|c|c|c|}
\hline \multicolumn{5}{|c|}{ CG } \\
\hline Data & $\Omega_{m}$ & $h$ & $\Delta \mathrm{BIC}$ & $\Delta \mathrm{AIC}$ \\
\hline $\mathrm{BAO}+\mathrm{CMB}+\mathrm{SNe}$ & $0.301 \pm 0.008$ & $0.677 \pm 0.007$ & 6.3 & 2.7 \\
\hline $\mathrm{BAO}+\mathrm{CMB}+\mathrm{SNe}+\mathrm{H}_{0}$ & $0.295 \pm 0.008$ & $0.684 \pm 0.007$ & 7.1 & 3.6 \\
\hline $\mathrm{BAO}+\mathrm{CMB}+\mathrm{SNe}+f \sigma_{8}$ & $0.302 \pm 0.008$ & $0.677 \pm 0.007$ & 6.0 & 2.3 \\
\hline $\mathrm{BAO}+\mathrm{CMB}+\mathrm{SNe}+f \sigma_{8}+\mathrm{H}_{0}$ & $0.295 \pm 0.008$ & $0.684 \pm 0.007$ & 7.1 & 3.4 \\
\hline
\end{tabular}

Table 15

Cosmological constraints for a selection of parameters for the Chaplygin gas model. The model parameter $A_{s}$ is found to be constrained tightly in the range $(0.999,1)$.

\begin{tabular}{lllll}
\hline \multicolumn{5}{c}{ GCG } \\
\hline Data & $\Omega_{m}$ & $\alpha(68 \% \mathrm{CL})$ & $\Delta$ BIC & $\Delta$ AIC \\
\hline BAO+CMB+SNe & $0.302 \pm 0.008$ & $0.0<\alpha<0.43$ & 10.0 & 4.7 \\
$\mathrm{BAO}+\mathrm{CMB}+\mathrm{SNe}+\mathrm{H}_{0}$ & $0.296_{-0.007}^{+0.007}$ & $0.0<\alpha<0.35$ & 10.9 & 5.6 \\
$\mathrm{BAO}+\mathrm{CMB}+\mathrm{SNe}+f \sigma_{8}$ & $0.302_{-0.008}^{+0.009}$ & $0.0<\alpha<0.38$ & 9.8 & 4.3 \\
$\mathrm{BAO}+\mathrm{CMB}+\mathrm{SNe}+f \sigma_{8}+\mathrm{H}_{0}$ & $0.295 \pm 0.008$ & $0.0<\alpha<0.35$ & 11.0 & 5.4 \\
\hline
\end{tabular}

Table 16

Cosmological constraints for a selection of parameters for the Generalized Chaplygin gas model.

\begin{tabular}{lllll}
\hline \multicolumn{5}{c}{ MCG } \\
\hline Data & $\alpha(68 \% \mathrm{CL})$ & $B(68 \% \mathrm{CL})$ & & \\
\hline $\mathrm{BAO}+\mathrm{CMB}+\mathrm{SNe}$ & $0.0<\alpha<0.28$ & $0.0<B<0.16$ & 13.8 & 6.7 \\
$\mathrm{BAO}+\mathrm{CMB}+\mathrm{SNe}+\mathrm{H}_{0}$ & $0.0<\alpha<0.33$ & $0.0<B<0.14$ & 14.7 & 7.6 \\
$\mathrm{BAO}+\mathrm{CMB}+\mathrm{SNe}+f \sigma_{8}$ & $0.0<\alpha<0.36$ & $0.0<B<0.15$ & 13.6 & 6.3 \\
$\mathrm{BAO}+\mathrm{CMB}+\mathrm{SNe}+f \sigma_{8}+\mathrm{H}_{0}$ & $0.0<\alpha<0.36$ & $0.0<B<0.16$ & 14.8 & 7.4 \\
\hline
\end{tabular}

Table 17

Cosmological constraints for a selection of parameters for the Modified Chaplygin gas model.

Table 18 and 19. These models do not relieve the tension of $\mathrm{H}_{0}$ with the other data.

From the $p$-value analysis, without $f \sigma_{8} \mathrm{IDE}_{2}$ performs similarly to $\Lambda \mathrm{CDM}$. However, it is able to fit the observed $f \sigma_{8}$ a little better than other models, which makes it one of the models with highest $p$-value when all data is included. The predicted observables, shown in Figure 19. demonstrate how its fit improves over other models for $f \sigma_{8}$. Clearly additional data from eBOSS and DESI on the growth of structure will tighten this model space further.

It has been suggested that a different dark sector interaction may be able to resolve the tension (Salvatelli et al. 2013, 2014, Costa et al. 2014; Murgia et al. 2016). As a simple test, we apply the geometrical probes to a particular coupled dark energy and dark matter model which has the interaction term proportional to the dark energy component $Q=\xi H \rho_{D E}$. In this model, a larger $\mathrm{H}_{0}$ can be obtained by increasing the coupling $\xi$, which implies a energy transfer from dark energy to dark matter (Murgia et al. 2016). Note that this model is not required to mimic the expansion as the $\Lambda \mathrm{CDM}$ model. Our result is presented in the bottom panel of Figure 18. The result shows that the improvement of the tension of $\mathrm{H}_{0}$ is marginal. The use of the non-compressed CMB data might resolve the tension by some amount; a more detailed analysis would be required to answer this question. The MCMC result of this model also presents a positive degeneracy between $h$ and the coupling constant $\xi$.

\subsection{Weakly-coupled canonical scalar field (WCSF)}

Figure 20 presents the constraints of the parameterizations for a weakly-coupled canonical scalar field (WCSF).
This model parameterizes the equation of state $w(a)$ at late times instead of writing down explicitly the potential energy of the field. Therefore various quintessence potentials can be mapped into the same parameter space. The figure shows constraints for one and two-parameter trajectories respectively. The constraints include $\Lambda \mathrm{CDM}$, which corresponds to setting all parameters $\left(\epsilon_{\infty}, \epsilon_{s}, \zeta_{s}\right)$ to be 0 , though allow some departure from it. Like Planck Collaboration et al. 2015b, we also find that the constraint on $\epsilon_{s}$ for the two-parameter case is tighter than the one-parameter case because the parameters are correlated. Table 20 and 21 present the results for this model.

The $p$-value test of this model doesn't show significant difference from the $\Lambda \mathrm{CDM}$ model. The predicted observables are tightly constrained under this model, comparable to those found in Figure 7 for the non-flat $\Lambda \mathrm{CDM}$ model.

\subsection{Holographic dark energy (HDE, ADE, RDE)}

The constraints of the holographic dark energy models are displayed in Figure 21, and listed in Tables 22, 23 and 24

For the HDE model, the dependence of $h$ on the inclusion of $\mathrm{H}_{0}$ data is stronger than in the standard $\Lambda \mathrm{CDM}$ model. The constraint on $n$ is tight, a result previously pointed out (Li et al. 2013, Li et al. 2013, Zhang et al. 2014). The addition of the linear growth data does not change the constraints significantly, revealing the consistency of the geometrical probes and the dynamical probes for this model (Xu 2013 Zhang et al. 2015). Figure 22 shows the predicted observables for this model, which show that although they are not currently ruled out, this class of models can be ruled out by future experiments. 


\begin{tabular}{|c|c|c|c|c|}
\hline \multicolumn{5}{|c|}{ Coupled dark energy: Model I } \\
\hline Data & $\overline{\Omega_{m}}$ & $\bar{w}$ & $\Delta \mathrm{BIC}$ & $\Delta \mathrm{AIC}$ \\
\hline $\mathrm{BAO}+\mathrm{CMB}+\mathrm{SNe}+f \sigma_{8}$ & $0.310 \pm 0.015$ & $-1.01 \pm 0.02$ & 5.8 & 2.1 \\
\hline $\mathrm{BAO}+\mathrm{CMB}+\mathrm{SNe}+f \sigma_{8}+\mathrm{H}_{0}$ & $0.302 \pm 0.014$ & $-1.01 \pm 0.02$ & 6.9 & 3.2 \\
\hline
\end{tabular}

Table 18

Cosmological constraints for a selection of parameters for the coupled dark energy model (Model I).

\begin{tabular}{llllll}
\hline \multicolumn{7}{c}{ Coupled dark energy: Model II } \\
\hline Data & $\bar{\Omega}_{m}$ & $\bar{w}_{0}$ & $\bar{w}_{1}$ & $\Delta$ BIC & $\Delta$ AIC \\
\hline $\mathrm{BAO}+\mathrm{CMB}+\mathrm{SNe}+f \sigma_{8}$ & $0.335_{-0.028}^{+0.030}$ & $-1.05 \pm 0.04$ & $0.14_{-0.13}^{+0.16}$ & 8.7 & 3.2 \\
$\mathrm{BAO}+\mathrm{CMB}+\mathrm{SNe}+f \sigma_{8}+\mathrm{H}_{0}$ & $0.327_{-0.026}^{+0.030}$ & $-1.05 \pm 0.04$ & $0.14_{-0.12}^{+0.15}$ & 10.0 & 4.4 \\
\hline
\end{tabular}

Table 19

Cosmological constraints for a selection of parameters for the coupled dark energy model (Model II).

\begin{tabular}{lllll}
\hline \multicolumn{5}{c}{ WCSF $_{1 D}$ : one-parameter trajectory } \\
\hline Data & $\Omega_{m}$ & $\epsilon_{s}$ & $\Delta$ BIC & $\Delta$ AIC \\
\hline $\mathrm{BAO}+\mathrm{CMB}+\mathrm{SNe}$ & $0.304 \pm 0.010$ & $0.14 \pm 0.24$ & 5.9 & 2.4 \\
$\mathrm{BAO}+\mathrm{CMB}+\mathrm{SNe}+\mathrm{H}_{0}$ & $0.292 \pm 0.009$ & $-0.10 \pm-0.22$ & 6.8 & 3.2 \\
$\mathrm{BAO}+\mathrm{CMB}+\mathrm{SNe}+f \sigma_{8}$ & $0.304 \pm 0.010$ & $0.20_{-0.24}^{+0.23}$ & 5.9 & 2.3 \\
$\mathrm{BAO}+\mathrm{CMB}+\mathrm{SNe}+f \sigma_{8}+\mathrm{H}_{0}$ & $0.292 \pm 0.009$ & $-0.04 \pm 0.20$ & 7.3 & 3.6 \\
\hline
\end{tabular}

Table 20

Cosmological constraints for a selection of parameters for the weakly-coupled scalar field: one-parameter trajectory.

\begin{tabular}{|c|c|c|c|c|}
\hline \multicolumn{5}{|c|}{$\mathrm{WCSF}_{2 D}$ : two-parameter trajectory } \\
\hline Data & $\epsilon_{s}$ & $\epsilon_{\infty}(68 \% \mathrm{CL})$ & $\Delta \mathrm{BIC}$ & $\Delta \mathrm{AIC}$ \\
\hline $\mathrm{BAO}+\mathrm{CMB}+\mathrm{SNe}$ & $0.01_{-0.11}^{+0.22}$ & $<0.64$ & 9.7 & 4.4 \\
\hline $\mathrm{BAO}+\mathrm{CMB}+\mathrm{SNe}+\mathrm{H}_{0}$ & $-0.11_{-0.14}^{+0.08}$ & $<0.73$ & 9.6 & 4.3 \\
\hline $\mathrm{BAO}+\mathrm{CMB}+\mathrm{SNe}+f \sigma_{8}$ & $0.11_{-0.11}^{+0.17^{14}}$ & $<0.51$ & 9.8 & 4.3 \\
\hline $\mathrm{BAO}+\mathrm{CMB}+\mathrm{SNe}+f \sigma_{8}+\mathrm{H}_{0}$ & $-0.06_{-0.12}^{+0.08}$ & $<0.65$ & 11.1 & 5.6 \\
\hline
\end{tabular}

Table 21

Cosmological constraints for a selection of parameters for the weakly-coupled scalar field: two-parameter trajectory.

The importance of linear growth data increases somewhat for the agegraphic dark energy (ADE) model but is still a marginal effect. The ADE predictions for $f \sigma_{8}$ are more similar to $\Lambda$ CDM than HDE's predictions are; unlike HDE, the predicted observables from ADE given current constraints fully contain $\Lambda \mathrm{CDM}$ (and are very similar to those found for $\Lambda \mathrm{CDM}$ in Figure 7). An important difference between these two models is that the parameter $n$ in $\mathrm{ADE}$ is not constrained. Unlike other studies in the literature, we are not applying the initial condition of the ADE model to reduce the number of parameters, so both the matter fraction $\Omega_{m}$ and $n$ are constrained in the MCMC test (Zhang et al. 2007; Wei \& Cai 2008, Li et al. 2009). In this case, the large value of $n$ preferred by the data gives $\Lambda$ CDM-like evolution in this model. Further theoretical considerations would be required to limit $n$ to low values, and doing so would likely rule out this model (Neupane 2009).

The bottom panel of Figure 21 displays the constraints of the Ricci dark energy model (RDE). The $p$-value test from the last section shows that there is significant incompatibility between this model and the data, which implies that this model is not favored. The constraints of the parameters also reveal tensions in $\alpha$ among different data combinations. This behavior is also found in the sDGP model, which is also not favored in the $p$-value test. Figure 23 shows the predicted observables under the best fit RDE model; both the expansion rate and the growth rate measurements clearly exclude it.

In HDE and RDE models, the degeneracy between $\Omega_{m}$ and $h$ is found to be similar to that of $\Lambda \mathrm{CDM}$. Therefore the unsolved tension of $\mathrm{H}_{0}$ also indicates a $1 \sigma$ disagreement in $\Omega_{m}$ and $>1.5 \sigma$ disagreement for $h$ among the datasets. However for ADE model, this tension is relaxed to be $0.5 \sigma$ for $\Omega_{m}$ and $1.0 \sigma$ for $h$. Nevertheless, our $p$ value analysis shows that this improvement comes at the expense of fitting the other data sets so that it is not a much different $\chi^{2}$ than many other models, and that the extra parameters introduced mean that the $p$-value is not much better for ADE or HDE.

\subsection{Quintessence scalar field model}

Cosmological constraints on the quintessence scalar field have been considered by a number of authors (Samushia \& Ratra 2008, Chen \& Ratra 2011; Farooq \& Ratra 2013; Chiba et al. 2013 Pavlov et al. 2014: Paliathanasis et al. 2014; and references therein). Figure 24 shows the constraints on the two Quintessence scalar field models considered in this work: the power law potential and exponential potential. The results of these models show an anti-correlation between the potential parameter and the Hubble constant. When $\mathrm{H}_{0}$ data is excluded, the non-zero value of $n$ for Model I and $\lambda$ for Model II implies a deviation from the $\Lambda \mathrm{CDM}$ model, which can be seen by the green curves in the figure. The addition of $\mathrm{H}_{0}$ data reduces this deviation and the stan- 


\begin{tabular}{|c|c|c|c|c|}
\hline \multicolumn{5}{|c|}{$\mathrm{HDE}$} \\
\hline Data & $\Omega_{m}$ & $n$ & $\Delta \mathrm{BIC}$ & $\Delta \mathrm{AIC}$ \\
\hline $\mathrm{BAO}+\mathrm{CMB}+\mathrm{SNe}$ & $0.302 \pm 0.010$ & $0.80_{-0.07}^{+0.08}$ & 10.3 & 6.8 \\
\hline $\mathrm{BAO}+\mathrm{CMB}+\mathrm{SNe}+\mathrm{H}_{0}$ & $0.290 \pm 0.009$ & $0.73 \pm 0.06$ & 10.5 & 6.9 \\
\hline $\mathrm{BAO}+\mathrm{CMB}+\mathrm{SNe}+f \sigma_{8}$ & $0.302 \pm 0.010$ & $0.80 \pm 0.07$ & 8.9 & 5.3 \\
\hline $\mathrm{BAO}+\mathrm{CMB}+\mathrm{SNe}+f \sigma_{8}+\mathrm{H}_{0}$ & $0.290 \pm 0.008$ & $0.73_{-0.05}^{+0.06}$ & 9.6 & 5.9 \\
\hline
\end{tabular}

Table 22

Cosmological constraints for a selection of parameters for the holographic dark energy model.

\begin{tabular}{|c|c|c|c|c|}
\hline \multicolumn{5}{|c|}{$\mathrm{ADE}$} \\
\hline Data & $\Omega_{m}$ & $n(68 \% \mathrm{CL})$ & $\Delta \mathrm{BIC}$ & $\Delta \mathrm{AIC}$ \\
\hline $\mathrm{BAO}+\mathrm{CMB}+\mathrm{SNe}$ & $0.299 \pm 0.008$ & $>154$ & 6.1 & 2.6 \\
\hline $\mathrm{BAO}+\mathrm{CMB}+\mathrm{SNe}+\mathrm{H}_{0}$ & $0.296_{-0.008}^{+0.009}$ & $>126$ & 7.1 & 3.6 \\
\hline $\mathrm{BAO}+\mathrm{CMB}+\mathrm{SNe}+f \sigma_{8}$ & $0.298 \pm 0.008$ & $>134$ & 6.0 & 2.4 \\
\hline $\mathrm{BAO}+\mathrm{CMB}+\mathrm{SNe}+f \sigma_{8}+\mathrm{H}_{0}$ & $0.293 \pm 0.008$ & $>168$ & 7.4 & 3.7 \\
\hline
\end{tabular}

Table 23

Cosmological constraints for a selection of parameters for the agegraphic dark energy model. The constraint on $n$ is an artifact due to the cutoff at around 1000 in our MCMC test. A less conservative choice of the this cutoff may change the result a little, but the estimation of other parameters here is still robust.

\begin{tabular}{|c|c|c|c|c|}
\hline \multicolumn{5}{|c|}{$\mathrm{RDE}$} \\
\hline Data & $\Omega_{m}$ & $\alpha$ & $\Delta \mathrm{BIC}$ & $\Delta \mathrm{AIC}$ \\
\hline $\mathrm{BAO}+\mathrm{CMB}+\mathrm{SNe}$ & $0.299_{-0.010}^{+0.011}$ & $0.393_{-0.014}^{+0.015}$ & 29.5 & 26.0 \\
\hline $\mathrm{BAO}+\mathrm{CMB}+\mathrm{SNe}+\mathrm{H}_{0}$ & $0.288 \pm 0.009$ & $0.381 \pm 0.012$ & 29.4 & 25.9 \\
\hline $\mathrm{BAO}+\mathrm{CMB}+\mathrm{SNe}+f \sigma_{8}$ & $0.298 \pm 0.010$ & $0.379_{-0.013}^{+0.014}$ & 38.7 & 35.1 \\
\hline $\mathrm{BAO}+\mathrm{CMB}+\mathrm{SNe}+f \sigma_{8}+\mathrm{H}_{0}$ & $0.289 \pm 0.009$ & $0.370 \pm 0.012$ & 38.3 & 34.6 \\
\hline
\end{tabular}

Table 24

Cosmological constraints for a selection of parameters for the Ricci scalar dark energy model.

dard model is well within $1 \sigma$ confidence region. This result holds for both scalar field models; a difference is that the tension of $\mathrm{H}_{0}$ in the power-law model is tighter than the exponential model. Table 25 and 26 summarize the constraint on the parameters for the quintessence scalar field models.

The $p$-value is relatively high for these models, and remains so when $\mathrm{H}_{0}$ is included, but this is a relatively small difference from $\Lambda \mathrm{CDM}$ and does not signify a very interesting reduction of the tension with $\mathrm{H}_{0}$. The predicted observables for these models are very similar to those for $\Lambda$ CDM (Figure 7).

\subsection{QCD ghost dark energy and DGP cosmology}

The QCD ghost dark energy model and the DGP model have similar expressions of the cosmic expansion. The $p$-value test shows that these two models are highly disfavored by the data, but they have different reasons for their incompatibility with the observations.

According to the $p$-value, the QCD ghost dark energy model agrees with the data quite well when only the $\mathrm{BAO}, \mathrm{CMB}$ and $\mathrm{SNe}$ data are considered. The addition of the linear growth data or $\mathrm{H}_{0}$ reduces the $p$-value significantly. The change of $\Delta \chi^{2}$ from $\mathrm{H}_{0}$ is about twice the amount found for the $\Lambda$ CDM model, which results in a significant decrease of the $p$-value. The top panel of Figure 25 displays the constraints on the QCD ghost dark energy model. The inclusion or exclusion of $\mathrm{H}_{0}$ clearly has a strong effect on the best fit parameters. The figure also shows a large difference made by $f \sigma_{8}$. The addition of this data set changes the component contributed from spatial curvature by about $2 \%$, which is a source of the tension measured in the $p$-value.
The reasons for this become clear when we consider the predicted observables from this model, in Figure 26. Relative to $\Lambda$ CDM, the QCD ghost dark energy model eases several tensions within the BAO data while requiring only slight compromises with the SNe and CMB. It slightly exacerbates the disagreement with $\mathrm{H}_{0}$, and also with $f \sigma_{8}$. Taken together, these two combine to make the model untenable. With eBOSS and DESI measurements of expansion and growth of structure this model could be ruled out completely without reference to $\mathrm{H}_{0}$.

As a modified gravity theory with an extra dimension, the DGP model and its application to cosmology has been explored widely (Lombriser et al. 2009, Azizi et al. 2012; Shi et al. 2012; $\mathrm{Xu}$ 2014; Santos et al. 2016 $\mathrm{Xu}$ \& Zhang 2016 and references therein). The $p$-value test indicates that this model is not favored by the data and the disagreement is stronger than the QCD ghost model and Ricci dark energy model. The bottom panel of Figure 25 shows the constraints of the sDGP model. These constraints show clear tensions among different data sets. However, one important contribution to the $\chi^{2}$ is from the disagreement of DGP model with the CMB data alone, which implies that the expansion of the universe at early times is quite different than the prediction from the model. The combination with other data sets enhances the tension and results in a huge incompatibility between the model and observations.

The DGP model allows the existence of the vacuum energy, therefore the normal branch is also able to predict the current acceleration. However, the addition of the vacuum energy in both the normal branch and selfaccelerating branch just gives a $\Lambda$ CDM-like model, and the modification of gravity from the extra-dimension is 


\begin{tabular}{lllll}
\hline \multicolumn{5}{c}{ Quintessence scalar field: power law potential } \\
\hline Data & $\Omega_{m}$ & $n(68 \% \mathrm{CL})$ & $\Delta \mathrm{BIC}$ & $\Delta \mathrm{AIC}$ \\
\hline $\mathrm{BAO}+\mathrm{CMB}+\mathrm{SNe}$ & $0.312 \pm 0.008$ & $0.00<n<0.40$ & 5.5 & 3.7 \\
$\mathrm{BAO}+\mathrm{CMB}+\mathrm{SNe}+\mathrm{H}_{0}$ & $0.302_{-0.007}^{+0.008}$ & $0.00<n<0.22$ & 6.3 & 4.5 \\
$\mathrm{BAO}+\mathrm{CMB}+\mathrm{SNe}+f \sigma_{8}$ & $0.312 \pm 0.009$ & $0.00<n<0.42$ & 4.8 & 3.0 \\
$\mathrm{BAO}+\mathrm{CMB}+\mathrm{SNe}+f \sigma_{8}+\mathrm{H}_{0}$ & $0.301 \pm 0.008$ & $0.00<n<0.26$ & 6.3 & 4.4 \\
\hline
\end{tabular}

Table 25

Cosmological constraints for a selection of parameters for the quintessence scalar field with power law potential.

\begin{tabular}{lllll}
\hline \multicolumn{5}{c}{ Quintessence scalar field: exponential potential } \\
\hline Data & $\Omega_{m}$ & $\lambda(68 \% \mathrm{CL})$ & $\Delta \mathrm{BIC}$ & $\Delta$ AIC \\
\hline $\mathrm{BAO}+\mathrm{CMB}+\mathrm{SNe}$ & $0.307 \pm 0.009$ & $0.00<\lambda<0.59$ & 4.3 & 2.6 \\
$\mathrm{BAO}+\mathrm{CMB}+\mathrm{SNe}+\mathrm{H}_{0}$ & $0.299 \pm 0.008$ & $0.00<\lambda<0.43$ & 4.5 & 2.7 \\
$\mathrm{BAO}+\mathrm{CMB}+\mathrm{SNe}+f \sigma_{8}$ & $0.306 \pm 0.009$ & $0.00<\lambda<0.63$ & 4.2 & 2.4 \\
$\mathrm{BAO}+\mathrm{CMB}+\mathrm{SNe}+f \sigma_{8}+\mathrm{H}_{0}$ & $0.297_{-0.007}^{+0.008}$ & $0.00<\lambda<0.45$ & 4.6 & 2.8 \\
\hline
\end{tabular}

Table 26

Cosmological constraints for a selection of parameters for the quintessence scalar field with exponential potential.

a weak effect $(\mathrm{Xu} 2014)$. Thus, we do not explore this possibility here.

The constraints on the parameters for the QCD ghost and DGP model are summarized in Table 27 and 28

\subsection{3. $f(R)$ gravity}

The designer model of $f(R)$ gravity has the same expansion history as the standard $\Lambda \mathrm{CDM}$ model; therefore, constraints on it come in this work from the linear growth data. Because the growth in $f(R)$ gravity is scale-dependent (Pogosian \& Silvestri 2008), the modifications to structure occur for some wavenumbers given the scale $B$ (Linder 2009), we test this model using two scaling wavenumbers as representatives, $k=0.1 \mathrm{hMpc}^{-1}$ and $k=0.02 h \mathrm{Mpc}^{-1}$, as suggested in Huterer et al. (2015) (hereafter called the $f(R)_{1}$ and $f(R)_{2}$ model respectively).

Figure 27 shows the constraints on these two models, with and without the inclusion of $\mathrm{H}_{0}$. The two models give similar constraints on the matter fraction $\Omega_{m}$; the allowed region for the factor $B_{0}$ is different, and includes $B_{0}=0$ in both cases. For $k=0.1 h \mathrm{Mpc}^{-1}$, the constraint on the Compton wavelength parameter is $B_{0}<1.0 \times 10^{-3}$ at $95 \%$ confidence level, while for $k=0.02 h \mathrm{Mpc}^{-1}$, the constraint is twice as bad. This property does not depend much on the $\mathrm{H}_{0}$ data, since the parameter $B_{0}$ only affects the evolution of the perturbation in the designer model. Note that for the constraints on $B_{0}$, only the redshift space distortion data as a dynamical probe are used. Previous work using more dynamical probes, such as the galaxy correlations and cluster data, shows consistent results (Lombriser et al. 2012, Cataneo et al. 2015). The constraint on the matter fraction $\Omega_{m}$ and the Compton wavelength parameter $B_{0}$ at the two different scales are summarized in Table 29 and 30 .

Since the constraints limit this model to be close to $\Lambda \mathrm{CDM}$, we expect that the $p$-value test will yield comparable results to $\Lambda \mathrm{CDM}$. Indeed, the $p$-value test of $f(R)$ gravity model shows is marginally more consistent with the data than is the $\Lambda \mathrm{CDM}$ model when all the datasets are used, and somewhat less consistent when $\mathrm{H}_{0}$ is included. The predicted observables under this model are tightly constrained to be near $\Lambda \mathrm{CDM}$ (similar to Figure 7).

$$
\text { 4.14. } f(T) \text { gravity }\left(f(T)_{\mathrm{PL}}, f(T)_{\operatorname{Exp} 1}, f(T)_{\operatorname{Exp} 2},\right.
$$

Figure 28 displays the constraints on the four $f(T)$ gravity models considered in this paper. The first three models tend to the $\Lambda \mathrm{CDM}$ cosmology when their parameter $b$ approaches 0 . When $f \sigma_{8}$ is included in the constraints, there is a slight deviation from $b=0$ for $f(T)_{\mathrm{PL}}$ (Model I), but still less than $2 \sigma$. This deviation is apparently reduced when the $\mathrm{H}_{0}$ measurement is added. All of these models perform similarly to $\Lambda \mathrm{CDM}$ in the $p$-value tests, in some cases slightly worse or slightly better (the latter especially when $\mathrm{H}_{0}$ is excluded). In these models, the predicted observables are fairly well constrained, similar to non-flat $\Lambda$ CDM (Figure 7), with a little more freedom for the power-law $f(T)$ (Model III).

$f(T)_{\tanh }$ (Model IV) is the hyperbolic-tangent model, which has the same number of parameters as the other $f(T)$ models. However, this model does not reduce to the $\Lambda \mathrm{CDM}$ cosmology for any value of its parameters. The constraints clearly depend strongly on whether the $f \sigma_{8}$ data are included. Correspondingly, the $p$-values for Model IV are only somewhat worse than $\Lambda$ CDM when $f \sigma_{8}$ is included, but far worse when it is included. Due to its non-trivial modification of the linear perturbation function, this model shows a value of the perturbation amplitude $\sigma_{8}$ of around 1.1 with about a $2 \%$ uncertainty, much higher than indicated by the CMB. This $f(T)$ model is therefore firmly ruled out by the growth of structure data, as also pointed out by Nesseris et al. (2013). Figure 29 shows the predicted observables, which demonstrates clearly how the predictions for the growth of structure deviate from that observed.

Note that none of these four different $f(T)$ models relax the tension with $\mathrm{H}_{0}$ better than the $\Lambda \mathrm{CDM}$ model.

The parameter constraints for the models are presented in Tables 31 34

\subsection{Galileon cosmology: Tracker solution (GAL)}

The Galileon theory has solutions with different branches, including the model conformally (disformally) coupled to matter, general solution, tracker solution and so on. For numerical simplicity, we only consider the tracker solution in this paper, and refer the reader to the literature for a comprehensive discussion of the Galileon 


\begin{tabular}{lllll}
\hline \multicolumn{5}{c}{ QCD ghost dark energy model } \\
\hline Data & $\Omega_{k}$ & $\gamma$ & $\Delta$ BIC & $\Delta$ AIC \\
\hline $\mathrm{BAO}+\mathrm{CMB}+\mathrm{SNe}$ & $0.037 \pm 0.006$ & $1.30_{-0.05}^{+0.06}$ & 3.4 & -0.2 \\
$\mathrm{BAO}+\mathrm{CMB}+\mathrm{SNe}+\mathrm{H}_{0}$ & $0.039_{-0.005}^{+0.006}$ & $1.34 \pm 0.05$ & 6.4 & 2.9 \\
$\mathrm{BAO}+\mathrm{CMB}+\mathrm{SNe}+f \sigma_{8}$ & $0.018_{-0.005}^{+0.006}$ & $1.15 \pm 0.03$ & 15.3 & 11.6 \\
$\mathrm{BAO}+\mathrm{CMB}+\mathrm{SNe}+f \sigma_{8}+\mathrm{H}_{0}$ & $0.020 \pm 0.005$ & $1.18 \pm 0.03$ & 23.2 & 19.5 \\
\hline
\end{tabular}

Table 27

Cosmological constraints for a selection of parameters for the QCD ghost dark energy model.

\begin{tabular}{lllll}
\hline \multicolumn{4}{c}{ DGP } & \\
\hline Data & $\Omega_{m}$ & $h$ & $\Delta$ BIC & $\Delta$ AIC \\
\hline BAO+CMB+SNe & $0.335 \pm 0.009$ & $0.635 \pm 0.006$ & 36.4 & 34.7 \\
$\mathrm{BAO}+\mathrm{CMB}+\mathrm{SNe}+\mathrm{H}_{0}$ & $0.325_{-0.008}^{+0.009}$ & $0.646 \pm 0.006$ & 55.5 & 53.7 \\
$\mathrm{BAO}+\mathrm{CMB}+\mathrm{SNe}+f \sigma_{8}$ & $0.341 \pm 0.009$ & $0.631 \pm 0.006$ & 44.0 & 42.1 \\
$\mathrm{BAO}+\mathrm{CMB}+\mathrm{SNe}+f \sigma_{8}+\mathrm{H}_{0}$ & $0.331 \pm 0.008$ & $0.642 \pm 0.006$ & 65.4 & 63.6 \\
\hline
\end{tabular}

Table 28

Cosmological constraints for a selection of parameters for the DGP model.

\begin{tabular}{lllll}
\hline \multicolumn{5}{c}{$f(R)$ gravity: $k=0.1 h \mathrm{Mpc}^{-1}$} \\
\hline Data & $\Omega_{m}$ & $B_{0}(95 \% \mathrm{CL})$ & $\Delta \mathrm{BIC}$ & $\Delta$ AIC \\
\hline $\mathrm{BAO}+\mathrm{CMB}+\mathrm{SNe}+f \sigma_{8}$ & $0.300 \pm 0.008$ & $0<B_{0}<0.001$ & 3.8 & 2.0 \\
$\mathrm{BAO}+\mathrm{CMB}+\mathrm{SNe}+f \sigma_{8}+\mathrm{H}_{0}$ & $0.294 \pm 0.007$ & $0<B_{0}<0.001$ & 3.9 & 2.0 \\
\hline
\end{tabular}

Table 29

Cosmological constraints for a selection of parameters for the $f(R)$ gravity with $k=0.1 h \mathrm{Mpc}^{-1}$.

\begin{tabular}{lllll}
\hline \multicolumn{5}{c}{$f(R)$ gravity: $k=0.02 h \mathrm{Mpc}^{-1}$} \\
\hline Data & $\Omega_{m}$ & $B_{0}(95 \% \mathrm{CL})$ & $\Delta \mathrm{BIC}$ & $\Delta \mathrm{AIC}$ \\
\hline $\mathrm{BAO}+\mathrm{CMB}+\mathrm{SNe}+f \sigma_{8}$ & $0.300 \pm 0.008$ & $0<B_{0}<0.003$ & 3.8 & 2.0 \\
$\mathrm{BAO}+\mathrm{CMB}+\mathrm{SNe}+f \sigma_{8}+\mathrm{H}_{0}$ & $0.294 \pm 0.007$ & $0<B_{0}<0.003$ & 3.9 & 2.0 \\
\hline
\end{tabular}

Table 30

Cosmological constraints for a selection of parameters for the $f(R)$ gravity with $k=0.02 h \mathrm{Mpc}^{-1}$.

\begin{tabular}{lllll}
\hline \multicolumn{5}{c}{$f(T)$ gravity: Model I } \\
\hline Data & $\Omega_{m}$ & $b$ & $\Delta$ BIC & $\Delta$ AIC \\
\hline $\mathrm{BAO}+\mathrm{CMB}+\mathrm{SNe}$ & $0.304 \pm 0.009$ & $0.06_{-0.10}^{+0.09}$ & 3.2 & 1.5 \\
$\mathrm{BAO}+\mathrm{CMB}+\mathrm{SNe}+\mathrm{H}_{0}$ & $0.295 \pm 0.008$ & $-0.05_{-0.12}^{+0.10}$ & 3.7 & 1.9 \\
$\mathrm{BAO}+\mathrm{CMB}+\mathrm{SNe}+f \sigma_{8}$ & $0.304 \pm 0.009$ & $0.09_{-0.08}^{+0.06}$ & 2.1 & 0.2 \\
$\mathrm{BAO}+\mathrm{CMB}+\mathrm{SNe}+f \sigma_{8}+\mathrm{H}_{0}$ & $0.294_{-0.007}^{+0.008}$ & $0.01_{-0.09}^{+0.08}$ & 3.8 & 1.9 \\
\hline
\end{tabular}

Table 31

Cosmological constraints for a selection of parameters for the $f(T)$ gravity: power-law model.

\begin{tabular}{lllll}
\hline \multicolumn{5}{c}{$f(T)$ gravity: Model II } \\
\hline Data & $\Omega_{m}$ & $b(68 \% \mathrm{CL})$ & $\Delta \mathrm{BIC}$ & $\Delta \mathrm{AIC}$ \\
\hline $\mathrm{BAO}+\mathrm{CMB}+\mathrm{SNe}$ & $0.305 \pm 0.009$ & $0.0<b<0.20$ & 3.4 & 1.7 \\
$\mathrm{BAO}+\mathrm{CMB}+\mathrm{SNe}+\mathrm{H}_{0}$ & $0.296 \pm 0.007$ & $0.0<b<0.15$ & 3.8 & 2.0 \\
$\mathrm{BAO}+\mathrm{CMB}+\mathrm{SNe}+f \sigma_{8}$ & $0.303_{-0.009}^{+0.010}$ & $0.0<b<0.19$ & 3.3 & 1.5 \\
$\mathrm{BAO}+\mathrm{CMB}+\mathrm{SNe}+f \sigma_{8}+\mathrm{H}_{0}$ & $0.295_{-0.007}^{+0.008}$ & $0.0<b<0.15$ & 3.9 & 2.0 \\
\hline
\end{tabular}

Table 32

Cosmological constraints for a selection of parameters for the $f(T)$ gravity: exponential model.

\begin{tabular}{lllll}
\hline \multicolumn{5}{c}{$f(T)$ gravity: Model III } \\
\hline Data & $\Omega_{m}$ & $b(68 \% \mathrm{CL})$ & $\Delta$ BIC & $\Delta$ AIC \\
\hline BAO+CMB+SNe & $0.305_{-0.008}^{+0.009}$ & $0.0<b<0.27$ & 3.1 & 1.4 \\
$\mathrm{BAO}+\mathrm{CMB}+\mathrm{SNe}+\mathrm{H}_{0}$ & $0.297 \pm 0.007$ & $0.0<b<0.20$ & 3.8 & 2.0 \\
$\mathrm{BAO}+\mathrm{CMB}+\mathrm{SNe}+f \sigma_{8}$ & $0.305_{-0.009}^{+0.010}$ & $0.0<b<0.32$ & 2.6 & 0.8 \\
$\mathrm{BAO}+\mathrm{CMB}+\mathrm{SNe}+f \sigma_{8}+\mathrm{H}_{0}$ & $0.295_{-0.007}^{+0.008}$ & $0.0<b<0.22$ & 3.9 & 2.0 \\
\hline
\end{tabular}

Table 33

Cosmological constraints for a selection of parameters for the $f(T)$ gravity: the second exponential model. 


\begin{tabular}{lllll}
\hline \multicolumn{5}{c}{$f(T)$ gravity: Model IV } \\
\\
\hline Data & $\Omega_{m}$ & $n$ & $\Delta$ BIC & $\Delta$ AIC \\
\hline BAO+CMB+SNe & $0.297 \pm 0.008$ & $1.63 \pm 0.02$ & 6.7 & 4.9 \\
$\mathrm{BAO}+\mathrm{CMB}+\mathrm{SNe}+\mathrm{H}_{0}$ & $0.290 \pm 0.007$ & $1.61 \pm 0.02$ & 5.9 & 4.2 \\
$\mathrm{BAO}+\mathrm{CMB}+\mathrm{SNe}+f \sigma_{8}$ & $0.297 \pm 0.008$ & $1.69 \pm 0.02$ & 154.0 & 152.2 \\
$\mathrm{BAO}+\mathrm{CMB}+\mathrm{SNe}+f \sigma_{8}+\mathrm{H}_{0}$ & $0.288 \pm 0.007$ & $1.68 \pm 0.02$ & 159.2 & 157.3 \\
\hline
\end{tabular}

Table 34

Cosmological constraints for a selection of parameters for the $f(T)$ gravity: hyperbolic-tangent model. 

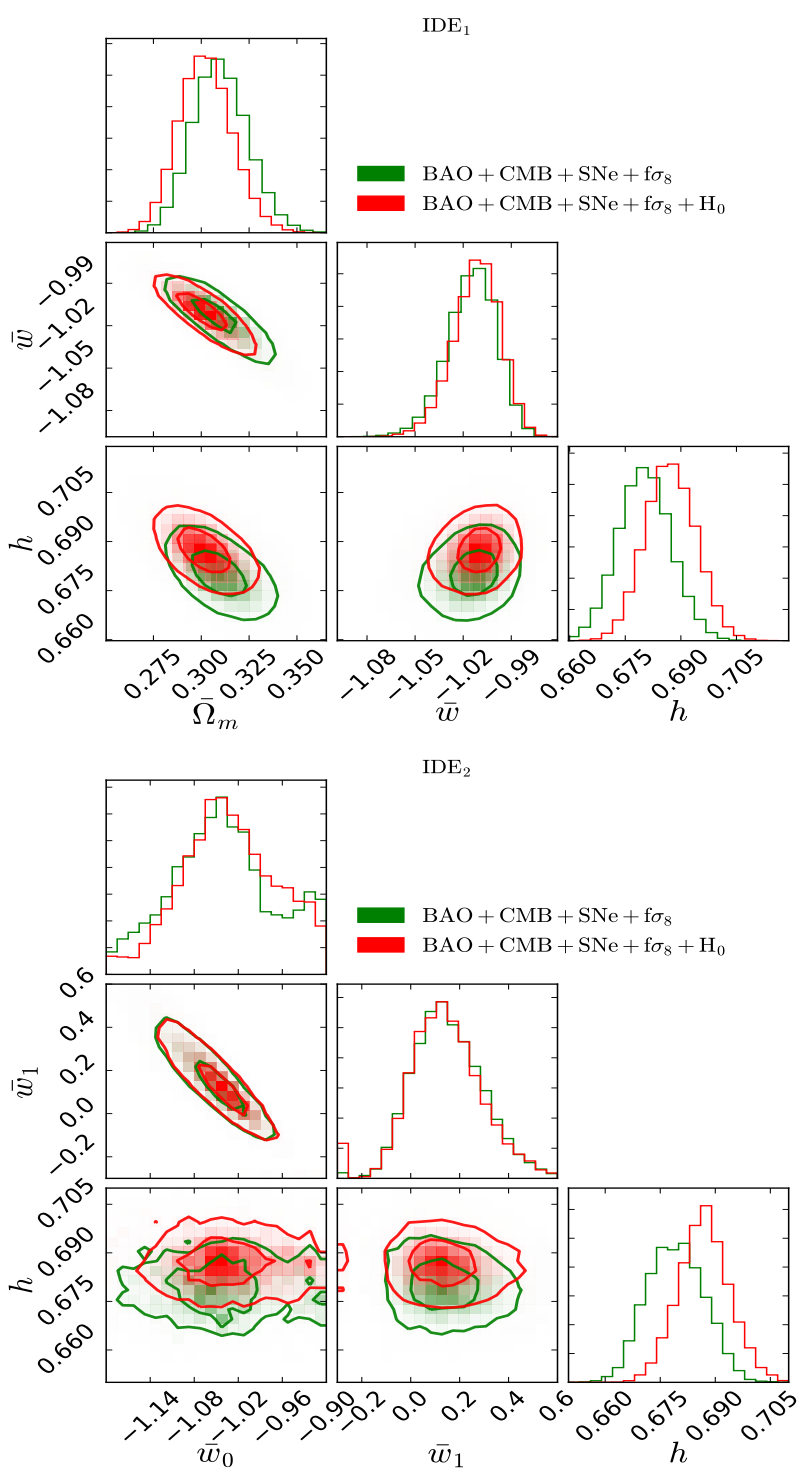

$\mathrm{IDE}_{3}$

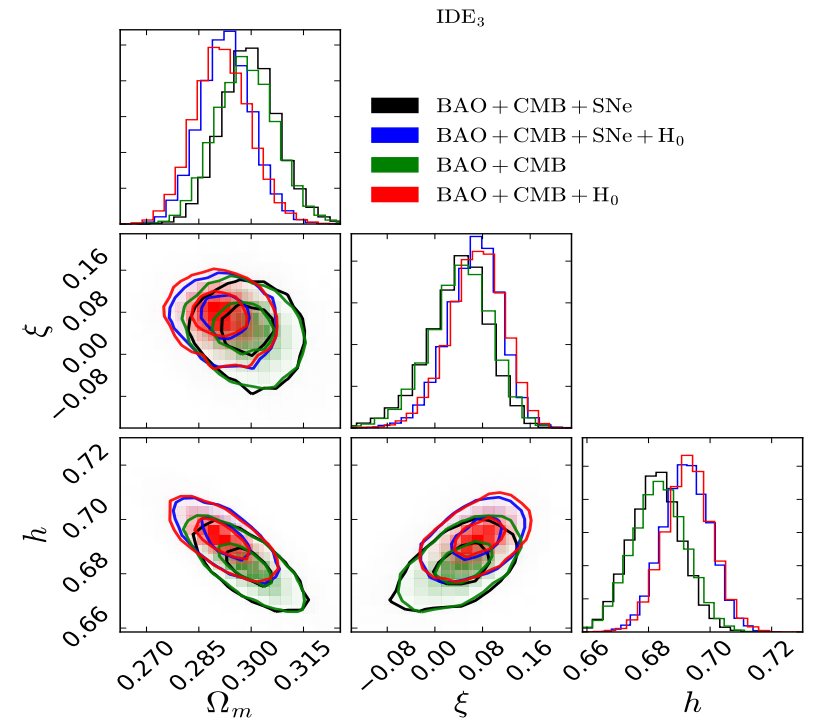

Figure 18. The $68.7 \%$ and $95.0 \%$ confidence regions of the parameters for two coupled dark energy models: Model I (Top panel) and Model II (Middle panel). The bottom panel shows a coupled dark energy and dark matter model which is not required to mimic the expansion of $\Lambda$ model. The diagonal panels show the one-dimensional probability distribution functions.
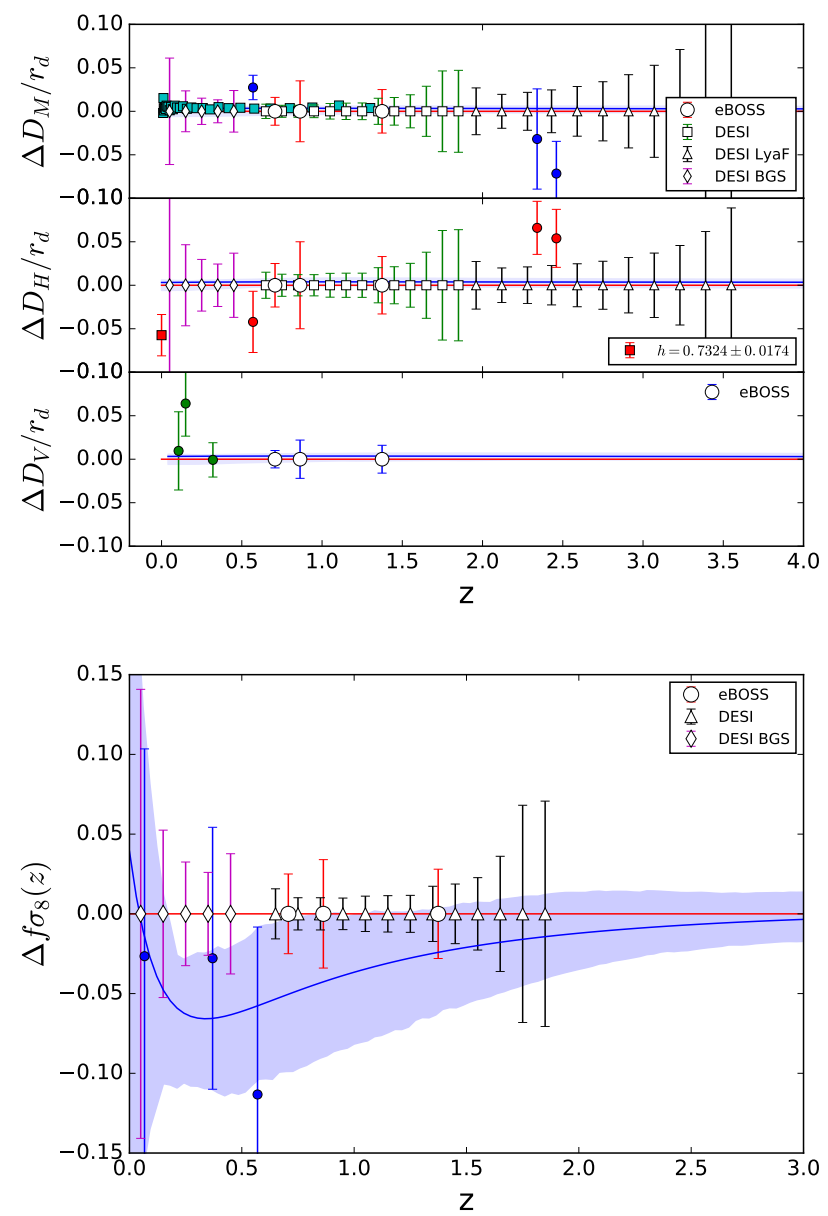

Figure 19. Similar to Figure 7 for the $\mathrm{IDE}_{2}$ model.

cosmology (Barreira et al. 2014ab; Brax et al. 2015 Neveu et al. 2016).

Figure 30 and Table 35 present the constraints on the tracker solution of the Galileon cosmology. The $p$-value test from the last section shows that this model is clearly ruled out by the data.

Figure 31 shows the predicted observables for this model. Interestingly, we see that in this model the tension with the Hubble constant $\mathrm{H}_{0}$ is resolved, so that the best fit $h$ does not depend much on whether $\mathrm{H}_{0}$ is included (as found, for example, by Barreira et al. (2014b)). However, this resolution comes at the expense of being able to fit well the BAO and especially the SNe data. As has been noted before, the growth of structure measurements are very different than $\Lambda$ CDM, but the expansion measurements are already enough to rule out the model completely.

The $p$-value test shows that the incompatibility between the data and the Galileon theory is only for the tracker solution. It is still possible to have more flexible descriptions of the evolution of the universe in this theoretical frame (Neveu et al. 2016).

\subsection{Kinetic gravity braiding model (KGBM, $\left.K G B M_{n=1}\right)$}

The kinetic gravity braiding model can be thought of as a generalization of the Galileon theory. The constraints 


\begin{tabular}{lllll}
\hline \multicolumn{5}{c}{ Galileon cosmology: tracker solution } \\
\hline Data & $\Omega_{m}$ & $h$ & $\Delta$ BIC & $\Delta$ AIC \\
\hline $\mathrm{BAO}+\mathrm{CMB}+\mathrm{SNe}$ & $0.272 \pm 0.007$ & $0.723_{-0.006}^{+0.007}$ & 29.6 & 27.8 \\
$\mathrm{BAO}+\mathrm{CMB}+\mathrm{SNe}+\mathrm{H}_{0}$ & $0.272 \pm 0.007$ & $0.723_{-0.005}^{+0.006}$ & 23.9 & 22.2 \\
$\mathrm{BAO}+\mathrm{CMB}+\mathrm{SNe}+f \sigma_{8}$ & $0.268 \pm 0.007$ & $0.727 \pm 0.007$ & 41.9 & 40.0 \\
$\mathrm{BAO}+\mathrm{CMB}+\mathrm{SNe}+f \sigma_{8}+\mathrm{H}_{0}$ & $0.268 \pm 0.006$ & $0.727 \pm 0.007$ & 36.4 & 34.5 \\
\hline
\end{tabular}

Table 35

Cosmological constraints for a selection of parameters for the tracker solution of the Galileon cosmology.
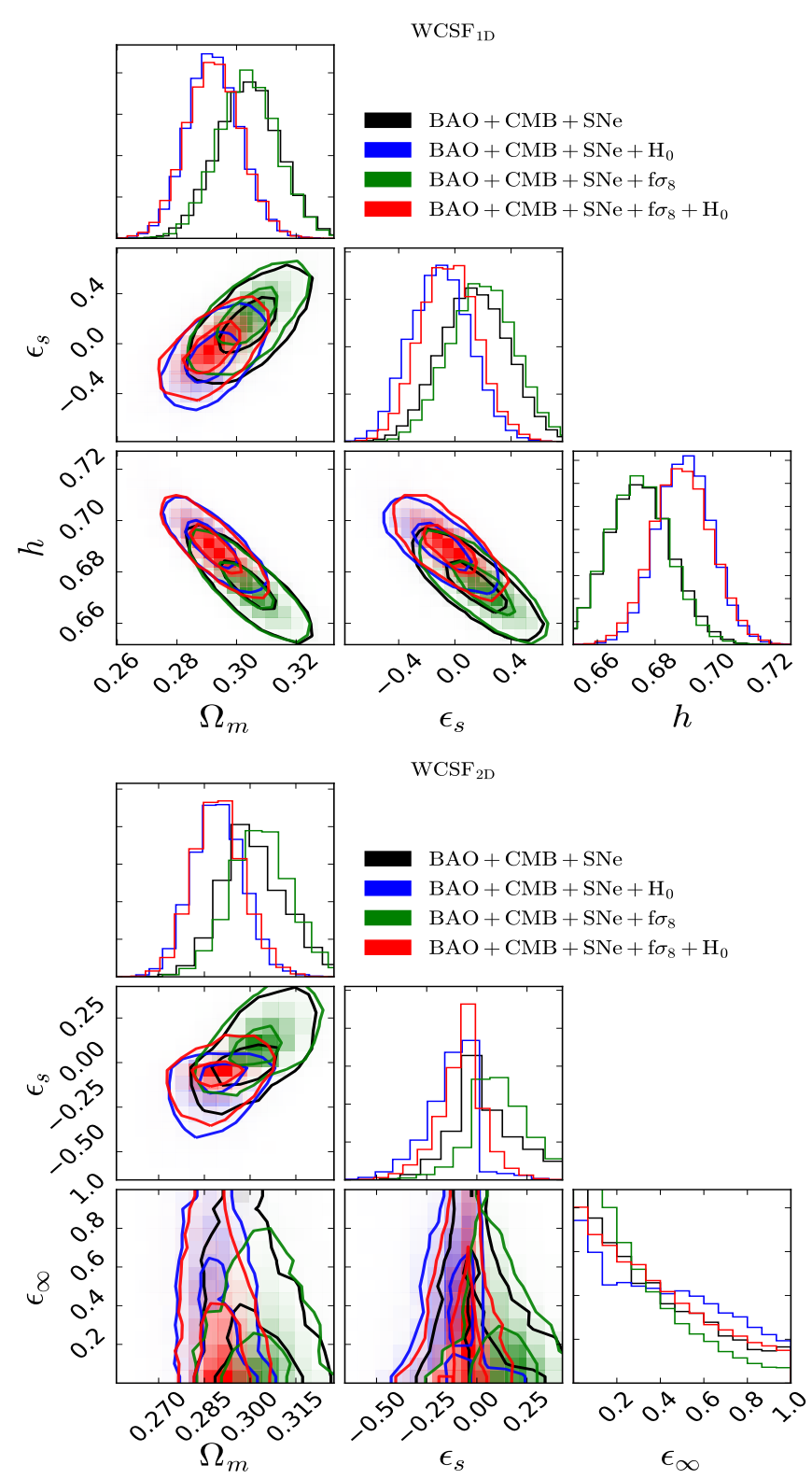

Figure 20. The $68.7 \%$ and $95.0 \%$ confidence regions of the parameters for the parameterization of the weakly-coupled canonical scalar field: one-parameter trajectory (Top panel) and twoparameter trajectory (Bottom panel) respectively. The diagonal panels show the one-dimensional probability distribution functions.

on this model are presented in Figure 32 . As pointed out in Section 2.1.2.5, the geometrical evolution of this model is equivalent to the power-law $f(T)$ theory (with a transformation relating $n$ and $b$ ). When $n$ approaches $\infty$, this model returns to the $\Lambda \mathrm{CDM}$ model. The constraint on $n$ has no useful upper bound for the data combinations considered here; in this way the KGBM model and the power-law $f(T)$ model give consistent results with each other.

The difference of these two theories is reflected by the linear growth data, because KGBM modifies the perturbation equation differently due to the time-dependent effective gravitational constant (Kimura et al. 2012). This results in a fit worse even than the power-law $f(T)$ model and therefore not favored by the linear growth data. The reason for the bad fit is that at early times, the effective gravitational constant $G_{\text {eff }}$ from the modification of the perturbation equation is nearly equal to $G$ in general relativity, and becomes larger than $G$ at late times (Kimura \& Yamamoto 2011). Figure 33 shows the predicted observables, revealing how KGBM can recover expansion very similar to $\Lambda \mathrm{CDM}$ but with a very different, and ruled out, rate of growth of structure.

The kinetic gravity braiding model has a special case when $n=1$, because its geometrical evolution is equivalent to the tracker solution of Galileon theory. However, these two models predict different growth of structure; even for $n=1$, the modified perturbation equation for the kinetic gravity braiding model is not identical to the tracker solution of the Galileon theory. The comparison of the $\mathrm{KGBM}_{n=1}$ predictions with linear growth data is however not better than the Galileon model; thus both of these two models are ruled out in the $p$-value test. Table 36 and 37 show the constraint results for these two cases of the kinetic gravity braiding model respectively.

\section{DISCUSSION AND CONCLUSION}

The underlying cause of cosmic acceleration is as yet unknown. The phenomenon has inspired a large variety of theoretical models and considerable expenditure of observational resources to create samples designed to test these models. The cosmological experiments have provided measurements of the history of expansion and growth of structure to a precision of percent-level or better, which strongly constrain the cosmological parameters. These developments motivate us to test the compatibility between the data and the various models for cosmic acceleration, in a consistent manner for all of them. We use measurements of the $\mathrm{CMB}, \mathrm{BAO}$ distances, $f \sigma_{8}$ using redshift space distortions, SNe Type Ia, and the local Hubble constant.

We have evaluated the models in three ways. First, we have calculated the $p$-value associated with a global $\chi^{2}$ statistic for each model, yielding the probability under the model of obtaining data in at least as much disagreement as the observations are. This approach allows us to determine which models can be ruled out by the data. However, it does not provide a framework for model selection - i.e. determining which of the models that are not ruled out should be favored. We have decided to avoid 


\begin{tabular}{|c|c|c|c|c|}
\hline \multicolumn{5}{|c|}{ KGBM } \\
\hline Data & $\Omega_{m}$ & $n(68 \% \mathrm{CL})$ & $\Delta \mathrm{BIC}$ & $\Delta \mathrm{AIC}$ \\
\hline $\mathrm{BAO}+\mathrm{CMB}+\mathrm{SNe}$ & $0.304 \pm 0.009$ & $>182$ & 3.2 & 1.5 \\
\hline $\mathrm{BAO}+\mathrm{CMB}+\mathrm{SNe}+\mathrm{H}_{0}$ & $0.295 \pm 0.008$ & $>138$ & 3.7 & 1.9 \\
\hline $\mathrm{BAO}+\mathrm{CMB}+\mathrm{SNe}+f \sigma_{8}$ & $0.296_{-0.006}^{+0.008}$ & $>180$ & 13.6 & 11.8 \\
\hline $\mathrm{BAO}+\mathrm{CMB}+\mathrm{SNe}+f \sigma_{8}+\mathrm{H}_{0}$ & $0.291_{-0.007}^{+0.008}$ & $>169$ & 13.9 & 12.0 \\
\hline
\end{tabular}

Table 36

Cosmological constraints for a selection of parameters for the kinetic gravity braiding model.

\begin{tabular}{lllll}
\hline \multicolumn{4}{c}{ KGBM with $n=1$} & \\
\hline Data & $\Omega_{m}$ & $h$ & $\Delta$ BIC & $\Delta$ AIC \\
\hline BAO+CMB+SNe & $0.272 \pm 0.007$ & $0.723_{-0.006}^{+0.007}$ & 29.6 & 27.8 \\
$\mathrm{BAO}+\mathrm{CMB}+\mathrm{SNe}+\mathrm{H}_{0}$ & $0.272 \pm 0.007$ & $0.723_{-0.005}^{+0.006}$ & 23.9 & 22.2 \\
$\mathrm{BAO}+\mathrm{CMB}+\mathrm{SNe}+f \sigma_{8}$ & $0.268 \pm 0.007$ & $0.727 \pm 0.007$ & 45.5 & 43.6 \\
$\mathrm{BAO}+\mathrm{CMB}+\mathrm{SNe}+f \sigma_{8}+\mathrm{H}_{0}$ & $0.268 \pm 0.006$ & $0.728 \pm 0.007$ & 40.0 & 38.1 \\
\hline
\end{tabular}

Table 37

Cosmological constraints for a selection of parameters for the tracker solution of the Galileon cosmology with $n=1$.

model selection because there is little basis for assigning any prior probability for each theoretical model (Efstathiou 2008). Second, we have constrained the parameters of each model using MCMC. Third, we have used the distribution of parameters from the MCMC to compute the predicted observables within each model space, given current constraints. These predicted observables yield insight into where tensions are arising with respect to the model and how future observations could constrain them.

The non-flat $\Lambda \mathrm{CDM}$ and its parametrized generalizations (XCDM, CPL, JBP, $w_{\text {Linear }}$, PolyCDM) exhibit some mild tension with the $\mathrm{CMB}, \mathrm{BAO}$, and $\mathrm{SNe}$, but not much with $f \sigma_{8}$. They all have difficulty explaining $\mathrm{H}_{0}$, which substantially reduces the $p$-value. In general, the less constrained models (e.g. PolyCDM) exhibit a larger variation in their predicted observables, but the range of behavior allowed with these model spaces, under current observational constraints, is relatively small.

Most other physical or parametric models we consider behave very similarly in general to the $\Lambda$ CDM-like ones. They do not exhibit specific redshift ranges that would be especially informative and for the most part when the expansion history measurements agree with $\Lambda$ CDM the growth of structure is predicted to as well.

However, some models behave in interesting ways. Several models are firmly ruled out by just the CMB, BAO, and SNe (Ricci Dark Energy, the Galileon tracker solution, and sDGP).

Adding $f \sigma_{8}$ rules out the $f(T)_{\text {tanh }}$ modified gravity model, rules out a particular class of the kinetic gravity braiding model (the $n=1$ solution), and puts great pressure even on the more general kinetic gravity braiding model and on the QCD ghost model. Holographic Dark Energy agrees very well with current $f \sigma_{8}$ measurements; it is a model that can reproduce $\Lambda$ CDM but could be ruled out strongly by future expansion rate or $f \sigma_{8}$ measurements from eBOSS and DESI.

Further adding $\mathrm{H}_{0}$ firmly rules out the QCD ghost model. In general, $\mathrm{H}_{0}$ adds substantial tension to all models. The Galileon tracker solution explains it pretty well, but fails on multiple other data sets, particularly the Type Ia SNe.

The $\mathrm{H}_{0}$ problem has been visited by many groups re- cently. By exploring an extended parameter space, Di Valentino et al. (2016, 2017) find that the dynamical dark energy component can mitigate the tension on $\mathrm{H}_{0}$ at some extent. A similar conclusion is also reached in Zhao et al. (2017) which employ an non-parametric approach in the modeling of dark energy, while the Bayesian evidence is not sufficient to favor over $\Lambda$ CDM model. A more physically well-motivated dark energy model is examined in Sola et al. (2017b a c) which assume the dark energy arises as running vacuum energy instead of a rigid cosmological constant. On the other hand, there are some studies on the $\mathrm{H}_{0}$ measurement. For instance, Zhang et al. (2017) present a blinded SNe Ia-based $\mathrm{H}_{0}$ determination and the result is found to be consistent with Riess et al. (2016). Wu \& Huterer (2017) calculate the sample variance of the local $\mathrm{H}_{0}$ measurement with a large-volume cosmological N-body simulation. The result indicates that the sample variance is not able to explain the current tension. Feeney et al. (2017) perform an analysis with a Bayesian hierarchical model for the local distance ladder and show that the odds against $\Lambda C D M$ is less dramatic than the common $3-\sigma$ discrepancy. Their work assesses the discrepancy by considering the full likelihood instead of a Gaussian or least square approximation, and pays particular attention to the Bayesian odds in order to perform model selection. In this work, we are not aiming at judging different models to select the ones that are favored. The frequentist $p$-value test, rather than evaluating models against each other directly, only tells us whether how unusual our data would be under each model.

As a general rule, among the models considered here, the redshift space distortion data on the linear $f \sigma_{8}$ does not appear to yield constraints specifically tuned to modified gravity models. sDGP and the Galileon tracker cosmology are ruled out already by expansion data, and the $f(R)$ designer model and several $f(T)$ models can reproduce the growth rate measurements. The modified gravity models where currently $f \sigma_{8}$ plays an important role are $f(T)_{\text {tanh }}$ and the kinetic gravity braiding model. Among the dark energy models, the QCD ghost model and the Holographic Dark Energy are most affected by $f \sigma_{8}$ (for HDE this power will likely only become evident in the next generation of experiments like eBOSS and 


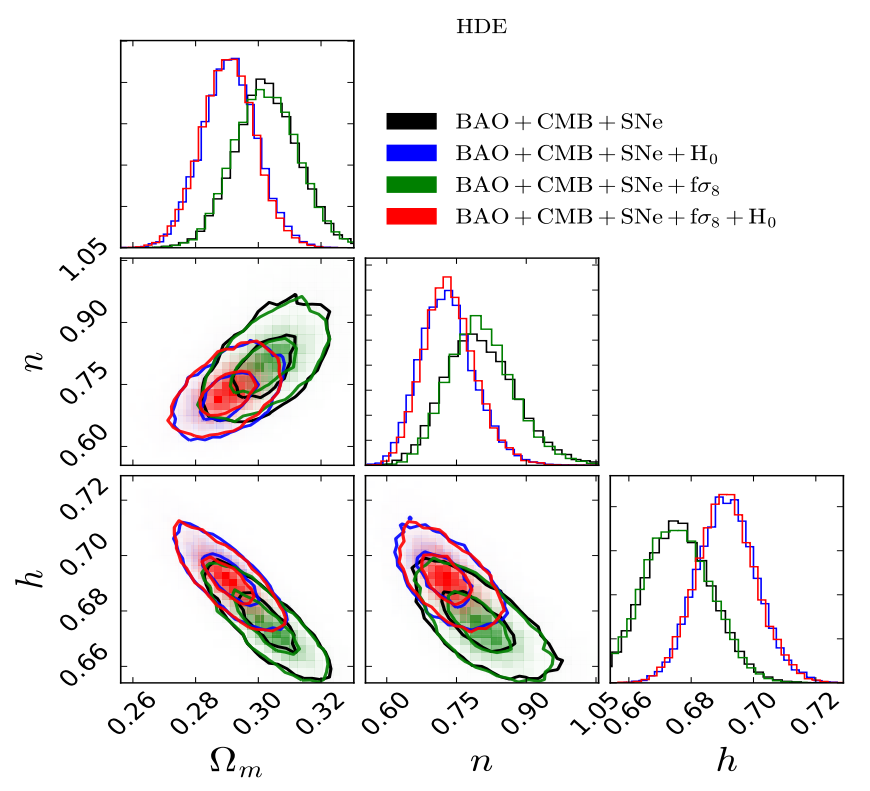

$\mathrm{ADE}$

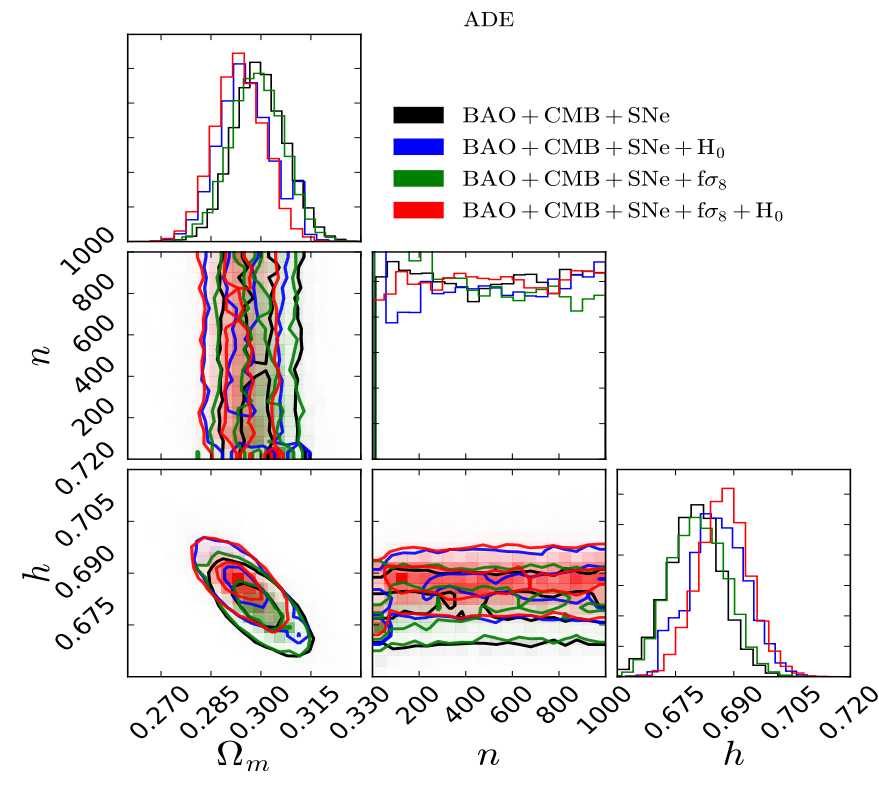

RDE

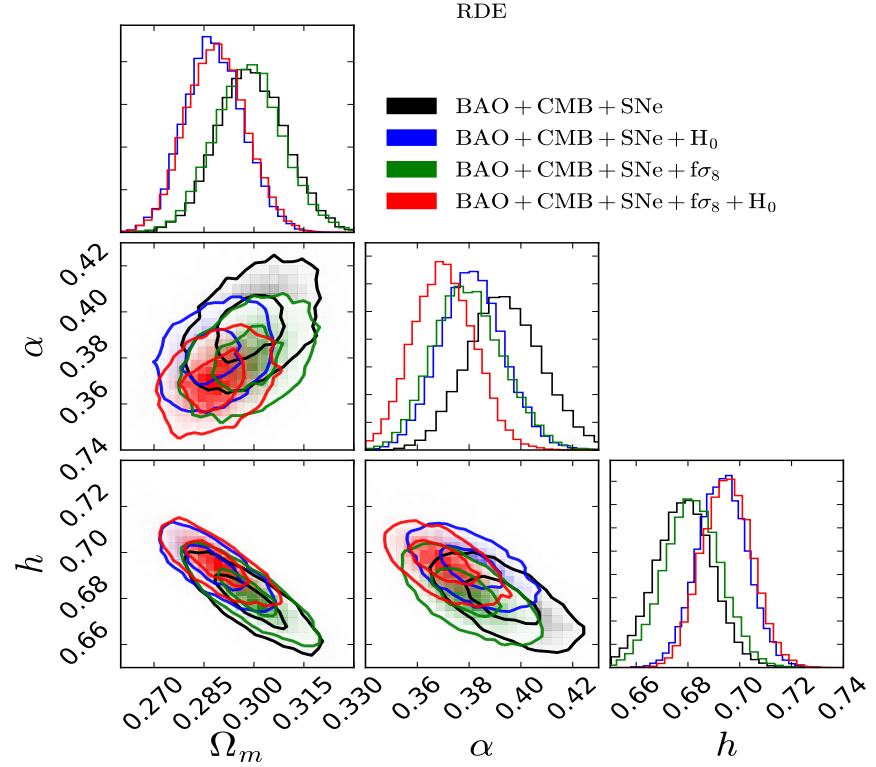

Figure 21. The $68.7 \%$ and $95.0 \%$ confidence regions of the parameters for the holographic dark energy model: $\operatorname{HDE}($ Top panel), ADE(Middle panel), RDE(Bottom panel). The diagonal panels show the one-dimensional probability distribution functions.
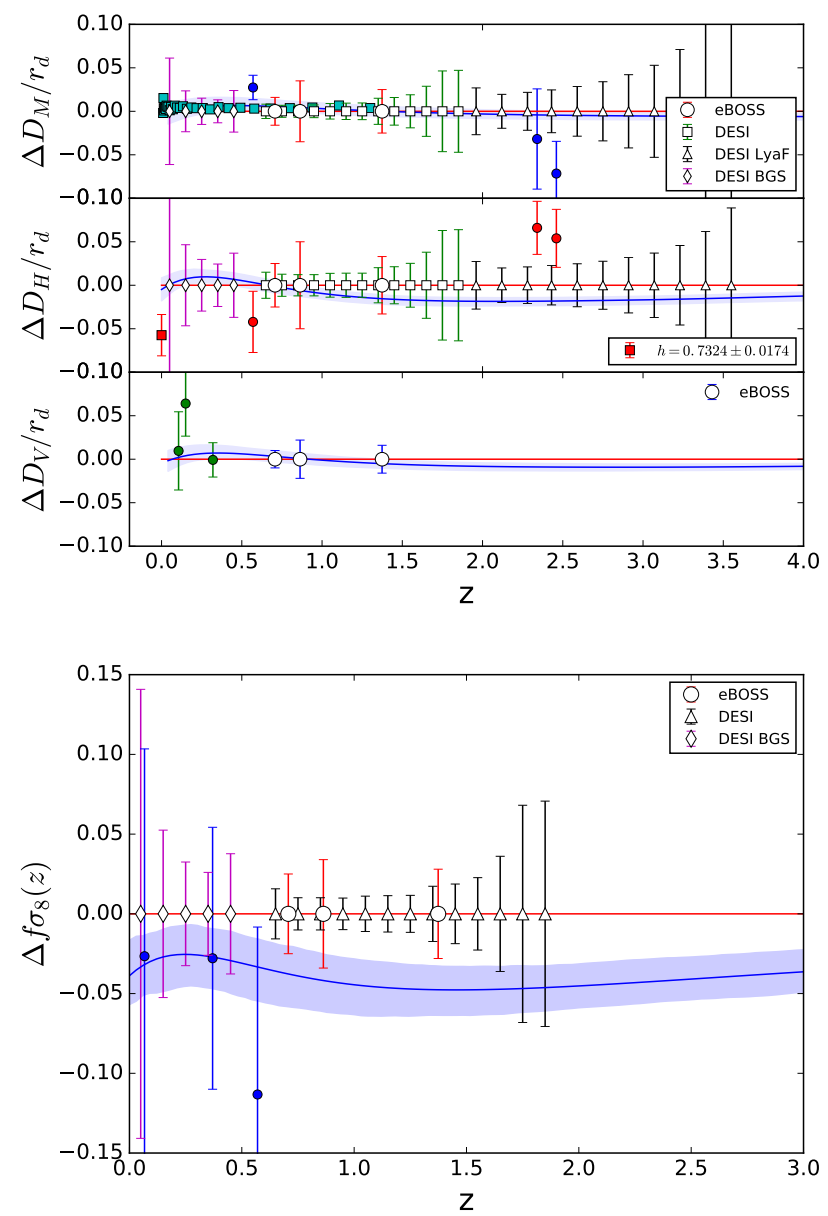

Figure 22. Similar to Figure 7 for the HDE model.

DESI).

The models we consider in this paper are based on phenomenological parameterizations of different quantities or physically motivated theoretical framework. In general, this parametric approach has clear behavior and prediction of observables for properly chosen parameters and initial conditions, at different redshifts and scales. The low number of parameters simplifies our analysis and interpretation. On the other hand, there are also numerous non-parametric or model-independent approaches in the modeling of cosmology, such as methodologies based on Principal Component Approach (PCA, Huterer \& Starkman 2003: Zhao et al. 2017), Gaussian Process (GP, Holsclaw et al. 2010; Seikel et al. 2012, Shafieloo et al. 2012) and so on. The non-parametric approach is much more flexible, and can capture possible features in the data that may be lost in particular parametric models, at the price of higher complexity and larger uncertainties and degeneracies in model parameters (Huterer \& Starkman 2003). In addition, the exact methodology may matter, since the best-fit of the reconstructed result may be inconsistent with different methods given the uncertainties. Therefore a detailed comparison between different non-parametric approaches with high dimensionality would be useful.

There are several limitations to our analysis that we 

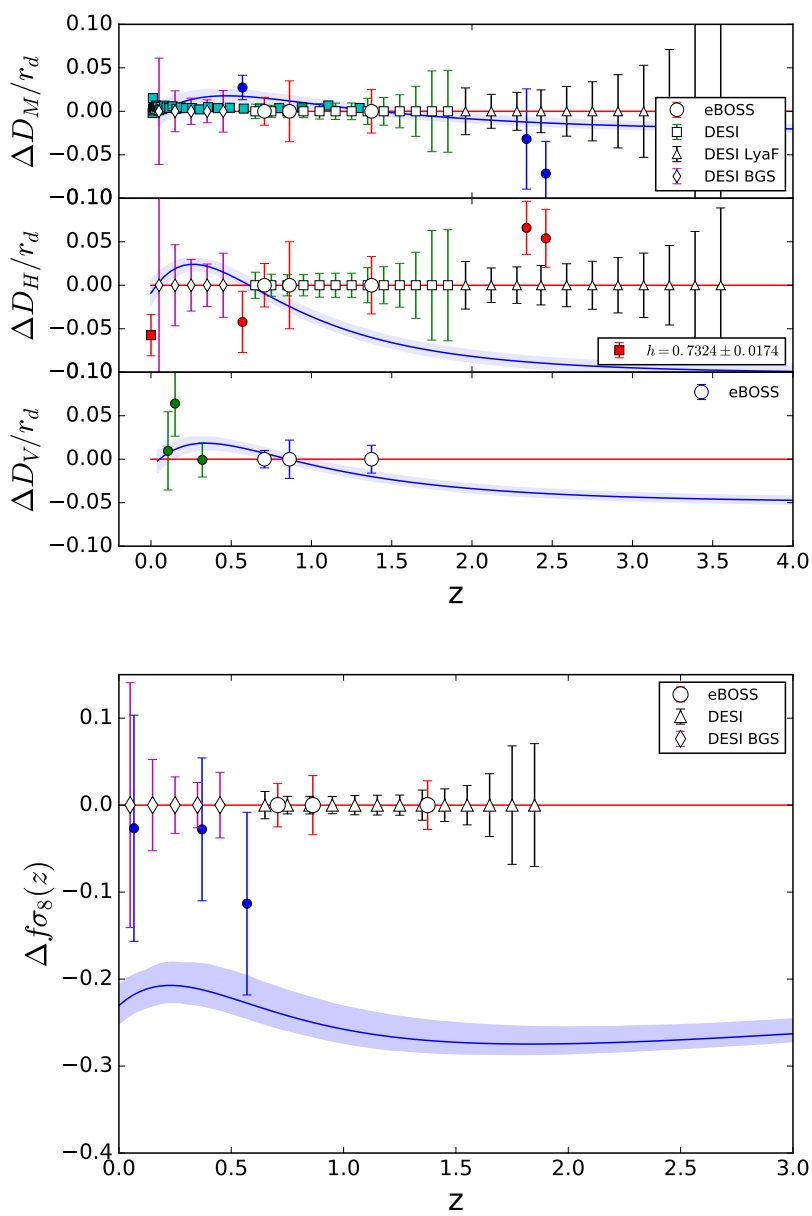

Figure 23. Similar to Figure 7 for the RDE model.

note here. First, we have included only a compressed version of the $\mathrm{CMB}$ data; there are further important constraints from the full Planck and other data sets. These constraints have informed the models we have chosen here (e.g. we have not considered the effects of extra relativistic degrees of freedom in the early universe) but not in a formal statistical way. Second, for the growth of structure data we have not included measurements that might probe scale dependence of gravity on large scales or of nonlinear scales. The predictions for these observables are much less straightforward to calculate for many of the models considered here.

We thank Joel Bronwnstein, Matthew Kleban, Boris Leistedt, Lucas Lombriser and Roman Scoccimarro for their kind helps and valuable comments. This work is supported by National Science Foundation NSF-AST1615997 and NSF-AST-1109432.

\section{REFERENCES}

Akaike, H. 1974, IEEE transactions on automatic control, 19, 716 Alam, S., Ata, M., Bailey, S., et al. 2016, ArXiv e-prints, arXiv: 1607.03155

Alcaniz, J. S., Dev, A., \& Jain, D. 2005, ApJ, 627, 26

Amendola, L. 2000, Phys. Rev. D, 62, 043511

Amendola, L., Gannouji, R., Polarski, D., \& Tsujikawa, S. 2007a, Phys. Rev. D, 75, 083504
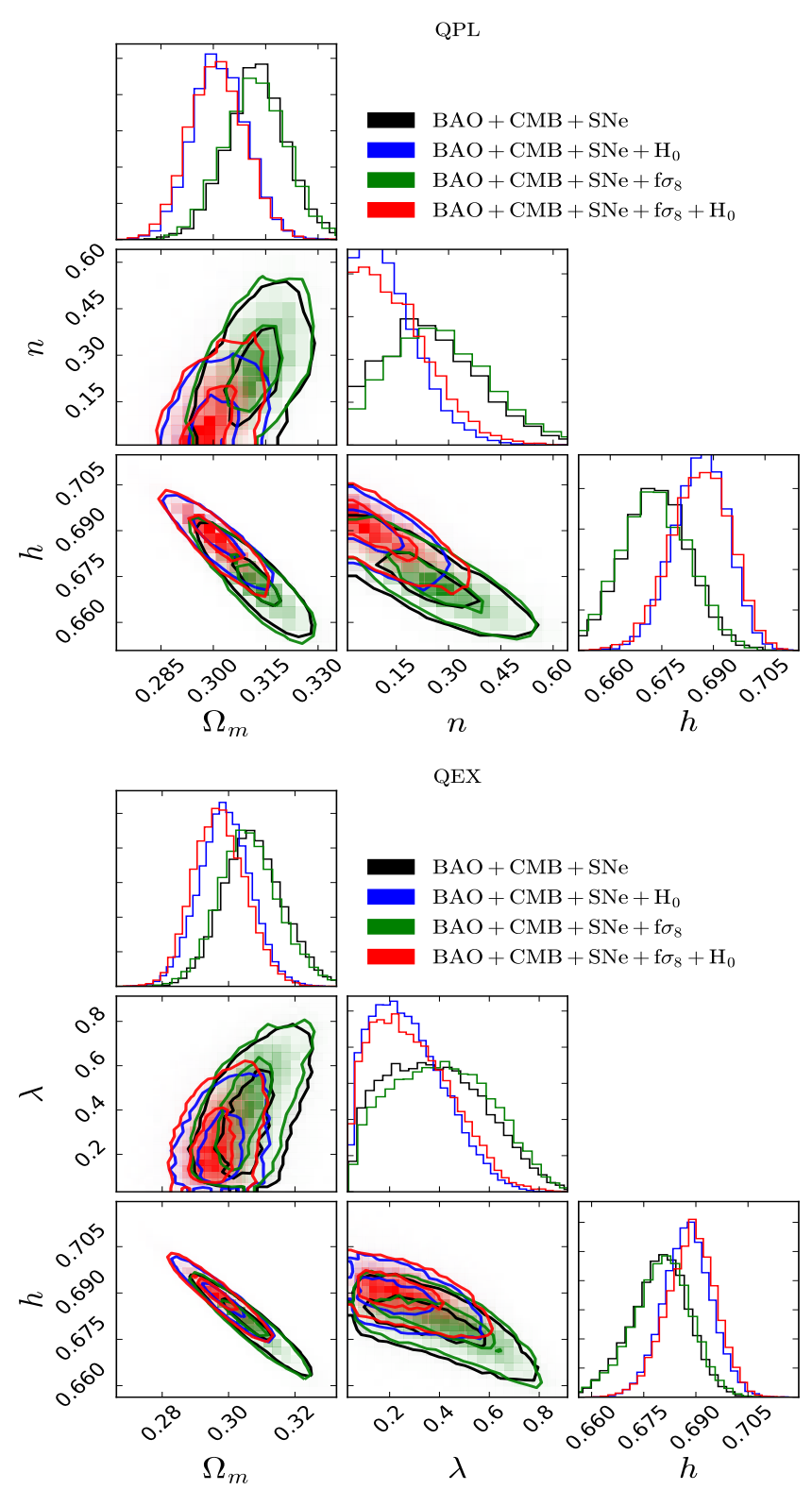

Figure 24. The $68.7 \%$ and $95.0 \%$ confidence regions of the parameters for the Quintessence scalar field model: Model I (Top panel), Model II (Bottom panel). The diagonal panels show the one-dimensional probability distribution functions.

Amendola, L., Polarski, D., \& Tsujikawa, S. 2007b, Physical Review Letters, 98, 131302

Anderson, L., Aubourg, É., Bailey, S., et al. 2014, MNRAS, 441, 24

Arcos, H. I., \& Pereira, J. G. 2004, International Journal of Modern Physics D, 13, 2193

Astier, P. 2001, Physics Letters B, 500, 8

Aubourg, É., Bailey, S., Bautista, J. E., et al. 2015, Phys. Rev. D, 92, 123516

Azizi, T., Movahed, M. S., \& Nozari, K. 2012, New Astronomy, 17,424

Bamba, K., Geng, C.-Q., Lee, C.-C., \& Luo, L.-W. 2011, Journal of Cosmology and Astroparticle Physics, 1, 021

Barreira, A., Li, B., Baugh, C. M., \& Pascoli, S. 2014a, Phys. Rev. D, 90, 023528

- 2014b, Journal of Cosmology and Astroparticle Physics, 8, 059

Barreiro, T., Copeland, E. J., \& Nunes, N. J. 2000, Phys. Rev. D, 61, 127301 


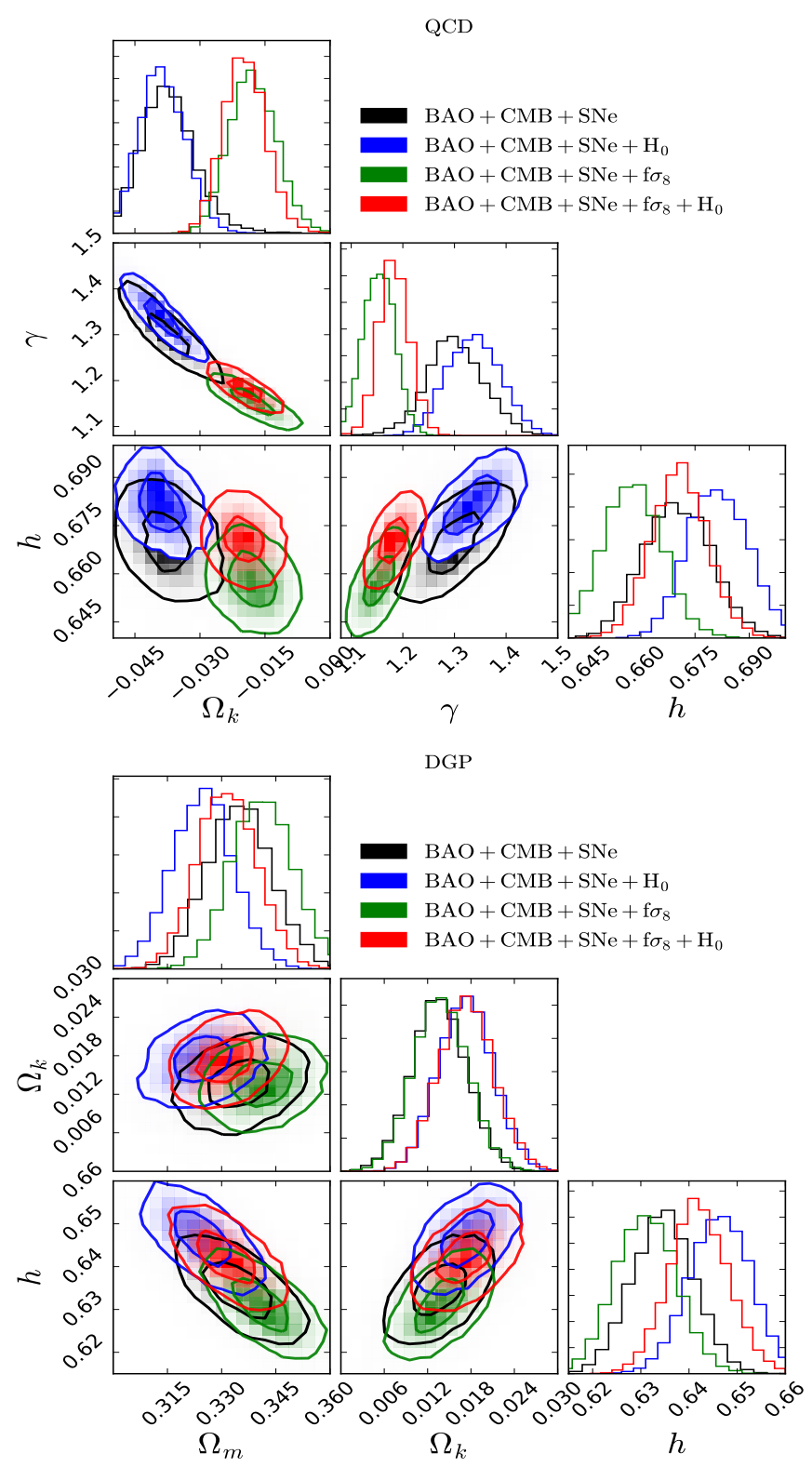

Figure 25. The $68.7 \%$ and $95.0 \%$ confidence regions of the parameters for the QCD Ghost dark energy model (Top panel) and self-accelerating DGP model (Bottom panel). The diagonal panels show the one-dimensional probability distribution functions.

Basilakos, S., Plionis, M., \& Lima, J. A. S. 2010, Phys. Rev. D, 82,083517

Bean, R., \& Doré, O. 2004, Phys. Rev. D, 69, 083503

Bean, R., Flanagan, É. É., Laszlo, I., \& Trodden, M. 2008, Phys. Rev. D, 78, 123514

Bekenstein, J. D. 1973, Phys. Rev. D, 7, 2333

—. 1981, Phys. Rev. D, 23, 287

Bekenstein, J. D. 1994, Phys. Rev. D, 49, 1912

Benaoum, H. B. 2002, ArXiv High Energy Physics - Theory e-prints, hep-th/0205140

Bengochea, G. R., \& Ferraro, R. 2009, Phys. Rev. D, 79, 124019

Bento, M. C., Bertolami, O., \& Sen, A. A. 2002, Phys. Rev. D, 66, 043507

Bertschinger, E., \& Zukin, P. 2008, Phys. Rev. D, 78, 024015

Betoule, M., Kessler, R., Guy, J., et al. 2014, A\&A, 568, A22

Beutler, F., Blake, C., Colless, M., et al. 2011, MNRAS, 416, 3017

—. 2012, MNRAS, 423, 3430

Beutler, F., Saito, S., Seo, H.-J., et al. 2014, MNRAS, 443, 1065
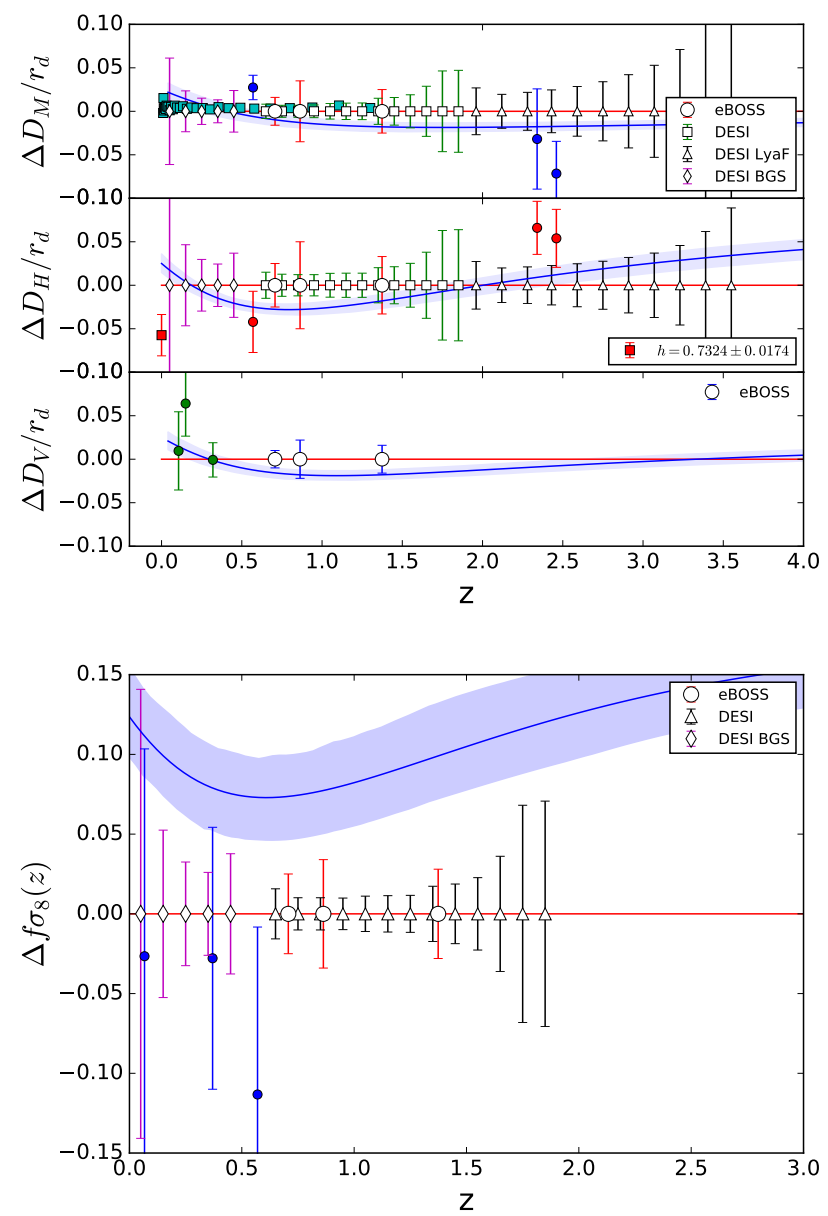

Figure 26. Similar to Figure 7 for the QCD model.

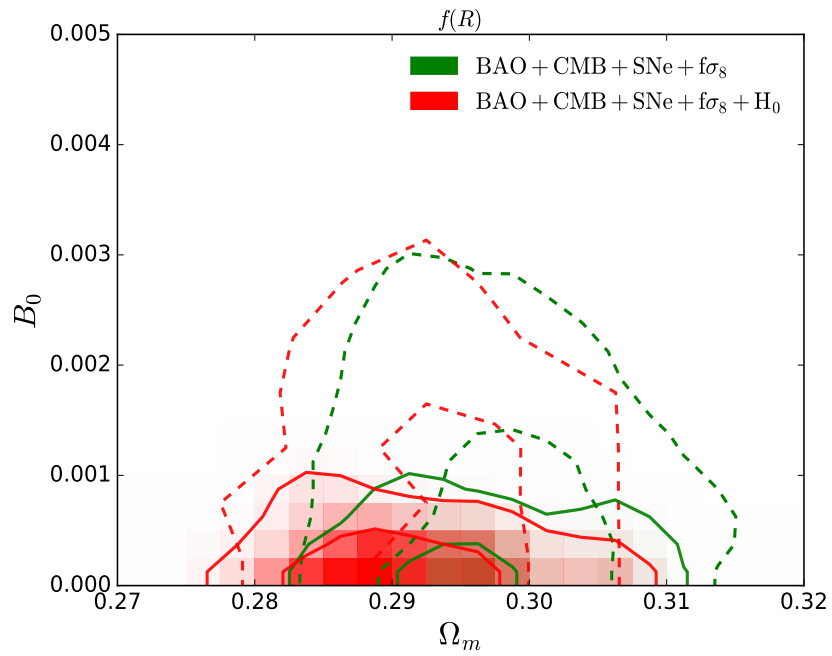

Figure 27. The $68.7 \%$ and $95.0 \%$ confidence regions of the parameters for the designer model of $f(R)$ gravity, solid line for $k=0.1$ and dashed line for $k=0.02$.

Bilić, N., Tupper, G. B., \& Viollier, R. D. 2002, Physics Letters $\mathrm{B}, 535,17$

Blake, C., \& Glazebrook, K. 2003, ApJ, 594, 665 

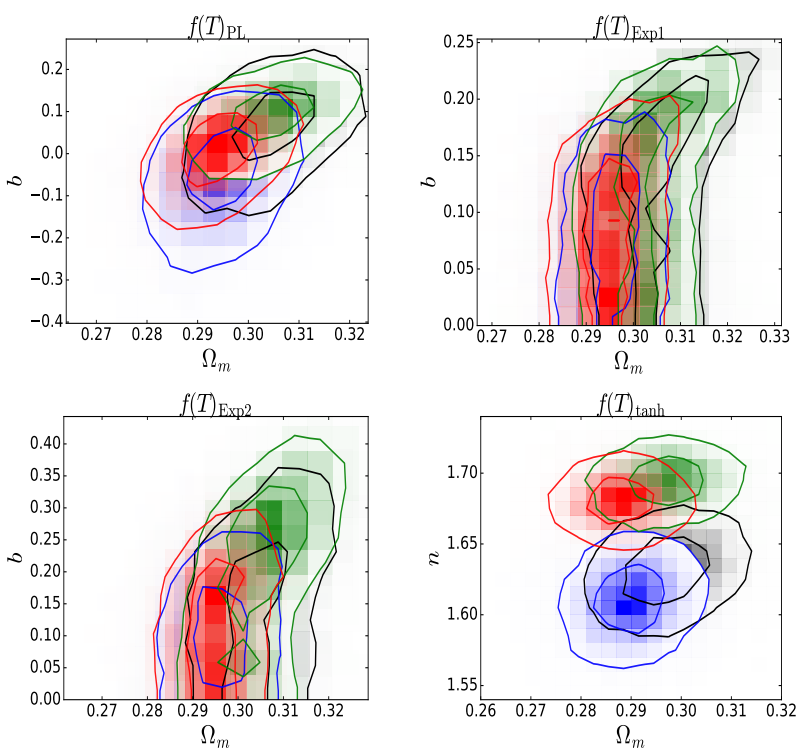

Figure 28. The $68.7 \%$ and $95.0 \%$ confidence regions of the parameters for the $f(T)$ gravity: Model I (power-law model, upper left), Model II (exponential model, upper right), Model III (another exponential model, lower left), Model IV (hyperbolic-tangent model, lower right). The diagonal panels show the one-dimensional probability distribution functions.

Bordag, M., Mohideen, U., \& Mostepanenko, V. M. 2001, Phys. Rep., 353, 1

Bordemann, M., \& Hoppe, J. 1993, Physics Letters B, 317, 315

Brax, P., Burrage, C., Davis, A.-C., \& Gubitosi, G. 2015, Journal of Cosmology and Astroparticle Physics, 3, 028

Cai, R.-G. 2007, Physics Letters B, 657, 228

Cai, R.-G., Tuo, Z.-L., Wu, Y.-B., \& Zhao, Y.-Y. 2012, Phys. Rev. D, 86, 023511

Cai, Y.-F., Capozziello, S., De Laurentis, M., \& Saridakis, E. N. 2015, ArXiv e-prints, arXiv:1511.07586

Caldera-Cabral, G., Maartens, R., \& Schaefer, B. M. 2009, Journal of Cosmology and Astroparticle Physics, 7, 027

Caldwell, R. R. 2002, Physics Letters B, 545, 23

Caldwell, R. R., Dave, R., \& Steinhardt, P. J. 1998, Physical Review Letters, 80, 1582

Caldwell, R. R., \& Kamionkowski, M. 2009, Annual Review of Nuclear and Particle Science, 59, 397

Caldwell, R. R., Kamionkowski, M., \& Weinberg, N. N. 2003, Physical Review Letters, 91, 071301

Capozziello, S., Cardone, V. F., Carloni, S., \& Troisi, A. 2003, International Journal of Modern Physics D, 12, 1969

Capozziello, S., \& Fang, L. Z. 2002, International Journal of Modern Physics D, 11, 483

Capozziello, S., Farooq, O., Luongo, O., \& Ratra, B. 2014, Phys. Rev. D, 90, 044016

Cataneo, M., Rapetti, D., Schmidt, F., et al. 2015, Phys. Rev. D, 92, 044009

Chen, Y., \& Ratra, B. 2011, Physics Letters B, 703, 406

Chevallier, M., \& Polarski, D. 2001, International Journal of Modern Physics D, 10, 213

Chiba, T., De Felice, A., \& Tsujikawa, S. 2013, Phys. Rev. D, 87, 083505

Chiba, T., Sugiyama, N., \& Nakamura, T. 1997, MNRAS, 289, L5

Chimento, L. P., Jakubi, A. S., Pavón, D., \& Zimdahl, W. 2003, Phys. Rev. D, 67, 083513

Chuang, C.-H., Prada, F., Cuesta, A. J., et al. 2013, MNRAS, 433, 3559

Cognola, G., Elizalde, E., Nojiri, S., Odintsov, S. D., \& Zerbini, S. 2005, Journal of Cosmology and Astroparticle Physics, 2, 010

Cohen, A. G., Kaplan, D. B., \& Nelson, A. E. 1999, Physical Review Letters, 82, 4971

Conley, A., Guy, J., Sullivan, M., et al. 2011, ApJS, 192, 1

Cooray, A. R., \& Huterer, D. 1999, ApJ, 513, L95
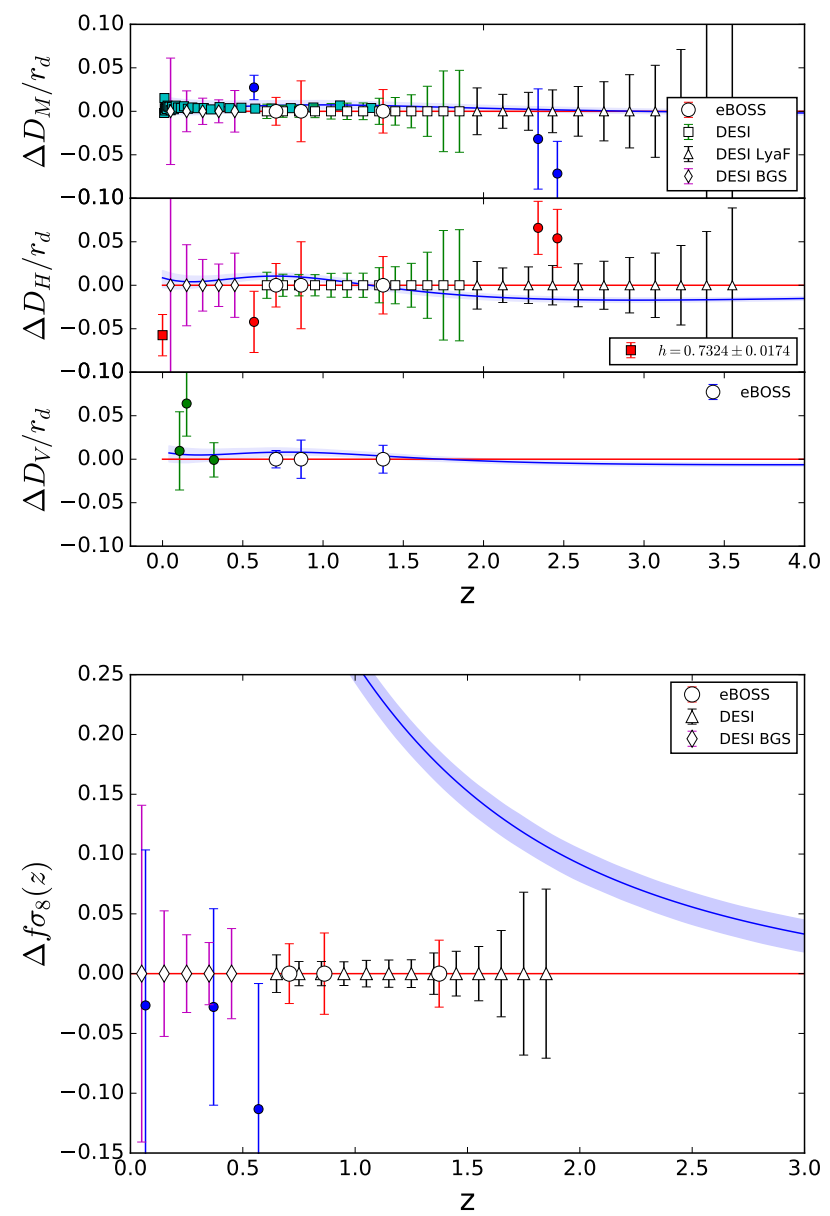

Figure 29. Similar to Figure 7 for the $f(T)_{\tanh }$ model.

Copeland, E. J., Liddle, A. R., \& Wands, D. 1998, Phys. Rev. D, 57,4686

Copeland, E. J., Sami, M., \& Tsujikawa, S. 2006, International Journal of Modern Physics D, 15, 1753

Costa, A. A., Xu, X.-D., Wang, B., Ferreira, E. G. M., \&

Abdalla, E. 2014, Phys. Rev. D, 89, 103531

Dalal, N., Abazajian, K., Jenkins, E., \& Manohar, A. V. 2001, Physical Review Letters, 87, 141302

Davis, T. M., Mörtsell, E., Sollerman, J., et al. 2007, ApJ, 666, 716

Dawson, K. S., Kneib, J.-P., Percival, W. J., et al. 2016, AJ, 151, 44

de Felice, A., Kase, R., \& Tsujikawa, S. 2011, Phys. Rev. D, 83, 043515

de Felice, A., \& Tsujikawa, S. 2010, Physical Review Letters, 105, 111301

—. 2011, Phys. Rev. D, 84, 124029

Deffayet, C., Deser, S., \& Esposito-Farèse, G. 2009a,

Phys. Rev. D, 80, 064015

Deffayet, C., Esposito-Farèse, G., \& Vikman, A. 2009b,

Phys. Rev. D, 79, 084003

Deffayet, C., Pujolàs, O., Sawicki, I., \& Vikman, A. 2010, Journal of Cosmology and Astroparticle Physics, 10, 026

Delubac, T., Bautista, J. E., Busca, N. G., et al. 2015, A\&A, 574, A59

DESI, C. 2016, arXiv:1611.00036

Di Valentino, E., Melchiorri, A., Linder, E. V., \& Silk, J. 2017, Phys. Rev. D, 96, 023523

Di Valentino, E., Melchiorri, A., \& Silk, J. 2016, Physics Letters B, 761, 242

Doran, M., \& Robbers, G. 2006, Journal of Cosmology and Astroparticle Physics, 6, 026 


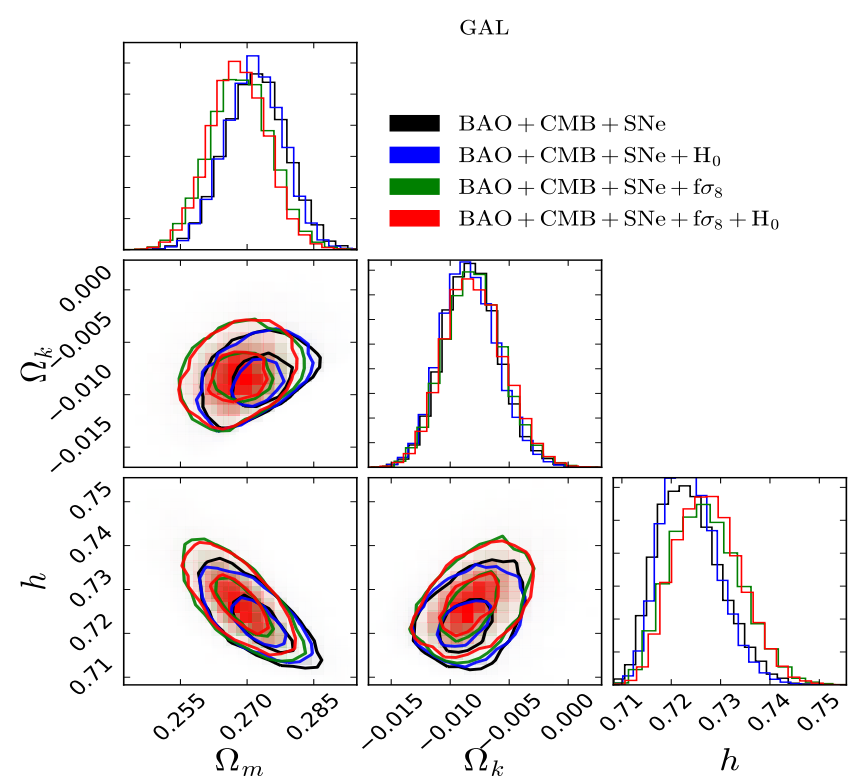

Figure 30. The $68.7 \%$ and $95.0 \%$ confidence regions of the parameters for the tracker solution of the Galileon cosmology. The diagonal panels show the one-dimensional probability distribution functions.

Dutta, K., \& Sorbo, L. 2007, Phys. Rev. D, 75, 063514

Dvali, G., Gabadadze, G., \& Porrati, M. 2000, Physics Letters B, 485, 208

Efstathiou, G. 2008, MNRAS, 388, 1314

Einstein, A. 1928, Riemann-Geometrie mit Aufrechterhaltung des Begriffes des Fernparallelismus (Wiley Online Library)

Eisenstein, D. J., Zehavi, I., Hogg, D. W., et al. 2005, ApJ, 633, 560

Faraoni, V., \& Dolgov, A. 2002, International Journal of Modern Physics D, 11, 471

Farooq, O., \& Ratra, B. 2013, Physics Letters B, 723, 1

Farrar, G. R., \& Peebles, P. J. E. 2004, ApJ, 604, 1

Fay, S. 2016, MNRAS, 460, 1863

Feeney, S. M., Mortlock, D. J., \& Dalmasso, N. 2017, ArXiv e-prints, arXiv:1707.00007

Ferraro, R., \& Fiorini, F. 2007, Phys. Rev. D, 75, 084031

-. 2008, Phys. Rev. D, 78, 124019

Font-Ribera, A., Kirkby, D., Busca, N., et al. 2014, Journal of Cosmology and Astroparticle Physics, 5, 027

Foreman-Mackey, D., Hogg, D. W., Lang, D., \& Goodman, J. 2013, PASP, 125, 306

Freese, K., \& Lewis, M. 2002, Physics Letters B, 540, 1

Frieman, J. A., Hill, C. T., Stebbins, A., \& Waga, I. 1995, Physical Review Letters, 75, 2077

Frith, W. J. 2004, MNRAS, 348, 916

Gao, C., Wu, F., Chen, X., \& Shen, Y.-G. 2009, Phys. Rev. D, 79, 043511

Gehrels, N. 1986, ApJ, 303, 336

Giannantonio, T., \& Melchiorri, A. 2006, Classical and Quantum Gravity, 23, 4125

Godłowski, W., \& Szydłowski, M. 2006, Physics Letters B, 642, 13

Goodman, J., \& Weare, J. 2010, Communications in applied mathematics and computational science, 5, 65

Gott, J. R., \& Slepian, Z. 2011, MNRAS, 416, 907

Hawking, S. W. 1976, Phys. Rev. D, 13, 191

Hayashi, K., \& Shirafuji, T. 1979, Phys. Rev. D, 19, 3524

Hinshaw, G., Larson, D., Komatsu, E., et al. 2013, The

Astrophysical Journal Supplement Series, 208, 19

Holsclaw, T., Alam, U., Sansó, B., et al. 2010, Physical Review Letters, 105, 241302

Hsu, S. D. H. 2004, Physics Letters B, 594, 13

Hu, W., \& Sawicki, I. 2007, Phys. Rev. D, 76, 064004

Huang, Q.-G., \& Li, M. 2004, Journal of Cosmology and Astroparticle Physics, 8, 013

Huang, Z., Bond, J. R., \& Kofman, L. 2011, ApJ, 726, 64
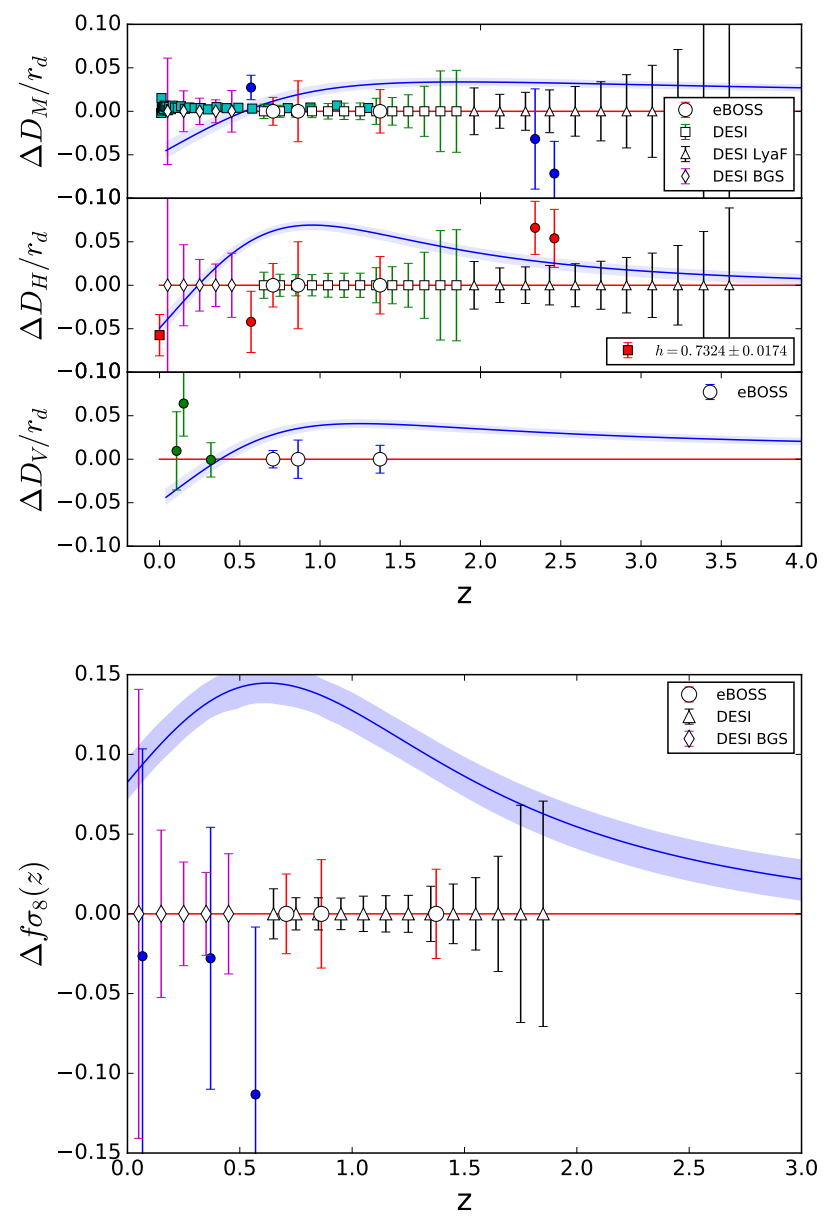

Figure 31. Similar to Figure 7 for the Galileon tracker model.

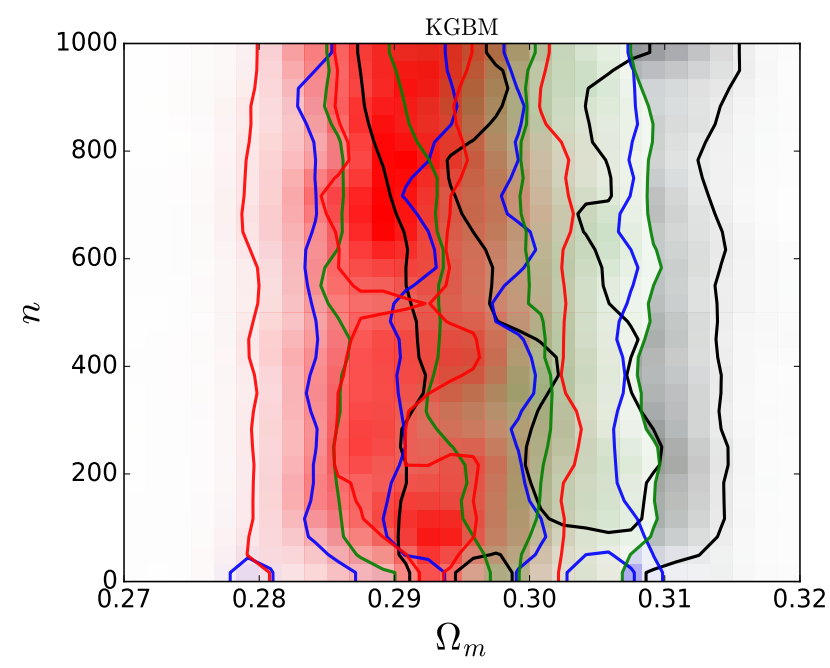

Figure 32. The $68.7 \%$ and $95.0 \%$ confidence regions of the parameters for the tracker solution of the Kinetic gravity braiding model. The diagonal panels show the one-dimensional probability distribution functions.

Huterer, D., \& Starkman, G. 2003, Physical Review Letters, 90, 03130 

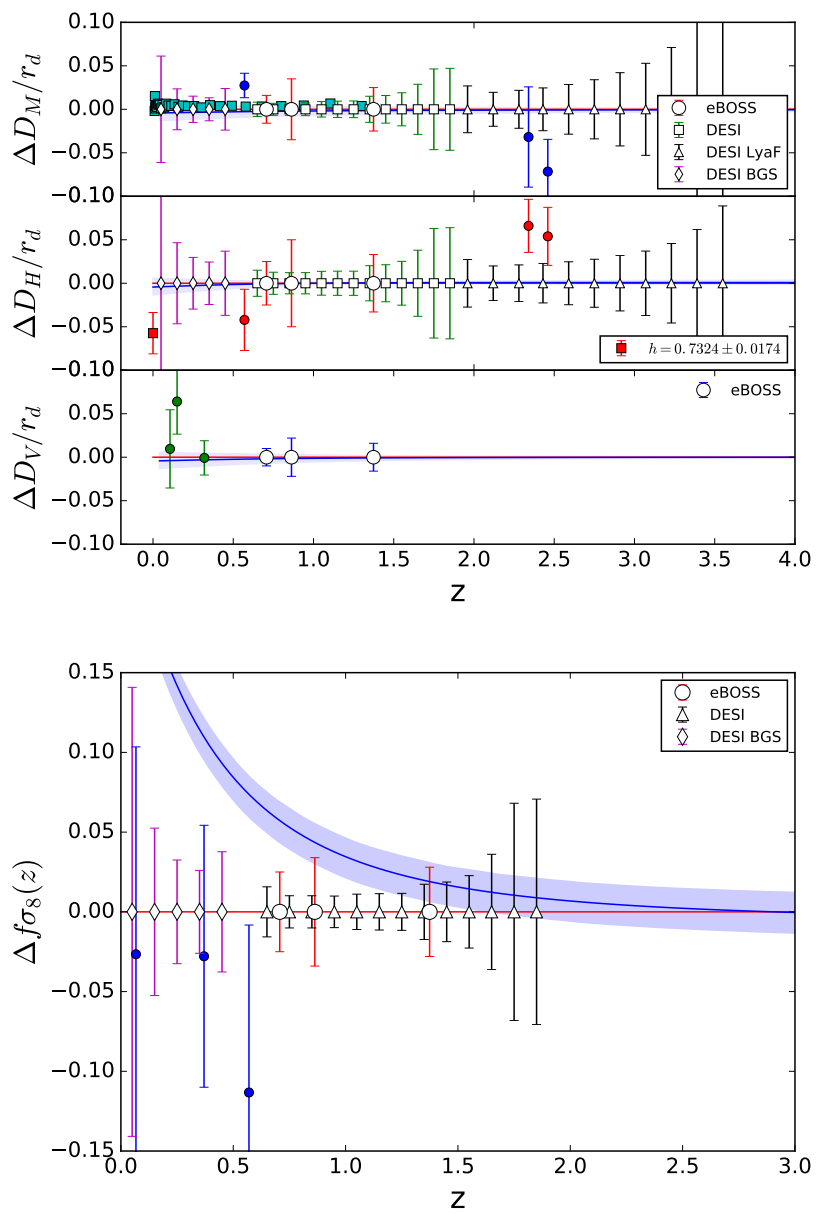

Figure 33. Similar to Figure 7 for the KGBM model.

Huterer, D., Kirkby, D., Bean, R., et al. 2015, Astroparticle Physics, 63, 23

Jassal, H. K., Bagla, J. S., \& Padmanabhan, T. 2005, MNRAS, 356, L11

Joyce, A., Jain, B., Khoury, J., \& Trodden, M. 2015, Phys. Rep., 568,1

Kaiser, N. 1987, MNRAS, 227, 1

Kamenshchik, A., Moschella, U., \& Pasquier, V. 2001, Physics Letters B, 511, 265

Karolyhazy, F. 1966, Il Nuovo Cimento A (1971-1996), 42, 390

Kawarabayashi, K., \& Ohta, N. 1980, Nuclear Physics B, 175, 477

Kawarabayashi, K., \& Ohta, N. 1981, Progress of Theoretical Physics, 66, 1789

Kimura, R., Kobayashi, T., \& Yamamoto, K. 2012, Phys. Rev. D, 85,123503

Kimura, R., \& Yamamoto, K. 2011, Journal of Cosmology and Astroparticle Physics, 4, 025

Levi, M., Bebek, C., Beers, T., et al. 2013, ArXiv e-prints, arXiv:1308.0847

Lewis, A., \& Bridle, S. 2002, Phys. Rev., D66, 103511

Lewis, A., Challinor, A., \& Lasenby, A. 2000, Astrophys. J., 538, 473

Li, B., \& Barrow, J. D. 2007, Phys. Rev. D, 75, 084010

Li, M. 2004, Physics Letters B, 603, 1

Li, M., Li, X.-D., Ma, Y.-Z., Zhang, X., \& Zhang, Z. 2013, Journal of Cosmology and Astroparticle Physics, 2013, 021

Li, M., Li, X.-D., Wang, S., \& Zhang, X. 2009, Journal of Cosmology and Astroparticle Physics, 6, 036

Li, Y.-H., Wang, S., Li, X.-D., \& Zhang, X. 2013, Journal of Cosmology and Astroparticle Physics, 2, 033

Liddle, A. R. 2004, MNRAS, 351, L49

Linder, E. V. 2003, Physical Review Letters, 90, 091301
—. 2008, Reports on Progress in Physics, 71, 056901

Linder, E. V. 2009, Phys. Rev. D, 80, 123528

-. 2010, Phys. Rev. D, 81, 127301

Lombriser, L., Hu, W., Fang, W., \& Seljak, U. 2009,

Phys. Rev. D, 80, 063536

Lombriser, L., Slosar, A., Seljak, U., \& Hu, W. 2012,

Phys. Rev. D, 85, 124038

Lu, J., Geng, D., Xu, L., Wu, Y., \& Liu, M. 2015, Journal of High Energy Physics, 2015, 1

Maluf, J. W. 1994, Journal of Mathematical Physics, 35

Maziashvili, M. 2007a, Physics Letters B, 652, 165

-. 2007b, International Journal of Modern Physics D, 16, 1531

Murgia, R., Gariazzo, S., \& Fornengo, N. 2016, Journal of

Cosmology and Astroparticle Physics, 4, 014

Nesseris, S., Basilakos, S., Saridakis, E. N., \& Perivolaropoulos, L. 2013, Phys. Rev. D, 88, 103010

Nesseris, S., de Felice, A., \& Tsujikawa, S. 2010, Phys. Rev. D, 82,124054

Neupane, I. P. 2009, Physics Letters B, 673, 111

Neveu, J., Ruhlmann-Kleider, V., Astier, P., et al. 2016, ArXiv e-prints, arXiv:1605.02627

Nicolis, A., Rattazzi, R., \& Trincherini, E. 2009, Phys. Rev. D, 79,064036

Nojiri, S., \& Odintsov, S. D. 2003, Phys. Rev. D, 68, 123512

Ohta, N. 1981, Progress of Theoretical Physics, 66, 1408

-. 2011, Physics Letters B, 695, 41

Paliathanasis, A., Tsamparlis, M., \& Basilakos, S. 2014, Phys. Rev. D, 90, 103524

Park, C.-G., Hwang, J.-C., Park, J., \& Noh, H. 2010,

Phys. Rev. D, 81, 063532

Paul, B. C., \& Thakur, P. 2013, Journal of Cosmology and Astroparticle Physics, 11, 052

Pavlov, A., Farooq, O., \& Ratra, B. 2014, Phys. Rev. D, 90, 023006

Peebles, P. J., \& Ratra, B. 2003, Reviews of Modern Physics, 75, 559

Peebles, P. J. E., \& Ratra, B. 1988, ApJ, 325, L17

Peebles, P. J. E., \& Yu, J. T. 1970, ApJ, 162, 815

Perlmutter, S., Aldering, G., Goldhaber, G., et al. 1999, ApJ, 517,565

Planck Collaboration, Ade, P. A. R., Aghanim, N., et al. 2014, A\&A, 571, A16

—. 2015a, ArXiv e-prints, arXiv:1502.01589

—. 2015b, ArXiv e-prints, arXiv:1502.01590

Pogosian, L., \& Silvestri, A. 2008, Phys. Rev. D, 77, 023503

Randall, L., \& Sundrum, R. 1999, Phys. Rev. Lett., 83, 4690

Randall, L., \& Sundrum, R. 1999, Physical Review Letters, 83, 3370

Rapetti, D., Blake, C., Allen, S. W., et al. 2013, Monthly Notices of the Royal Astronomical Society, 432, 973

Ratra, B., \& Peebles, P. J. E. 1988, Phys. Rev. D, 37, 3406

Reid, B. A., Samushia, L., White, M., et al. 2012, MNRAS, 426, 2719

Riess, A. G., Filippenko, A. V., Challis, P., et al. 1998, AJ, 116, 1009

Riess, A. G., Macri, L. M., Hoffmann, S. L., et al. 2016, ArXiv e-prints, arXiv:1604.01424

Rosenzweig, C., Schechter, J., \& Trahern, C. G. 1980, Phys. Rev. D, 21, 3388

Ross, A. J., Samushia, L., Howlett, C., et al. 2015, MNRAS, 449, 835

Sakharov, A. D. 1966, Sov. Phys. JETP, 22, 241

Sako, M., Bassett, B., Becker, A. C., et al. 2014, ArXiv e-prints, arXiv:1401.3317

Salvatelli, V., Marchini, A., Lopez-Honorez, L., \& Mena, O. 2013, Phys. Rev. D, 88, 023531

Salvatelli, V., Said, N., Bruni, M., Melchiorri, A., \& Wands, D. 2014, Physical Review Letters, 113, 181301

Samushia, L., Percival, W. J., \& Raccanelli, A. 2012, MNRAS, 420, 2102

Samushia, L., \& Ratra, B. 2008, ApJ, 680, L1

Santos, B., Chandrachani Devi, N., \& Alcaniz, J. S. 2016, ArXiv e-prints, arXiv:1603.06563

Sawicki, I., \& Hu, W. 2007, Phys. Rev. D, 75, 127502

Schwarz, G., et al. 1978, The annals of statistics, 6, 461

Scoccimarro, R. 2004, Phys. Rev. D, 70, 083007 
Seikel, M., Clarkson, C., \& Smith, M. 2012, Journal of Cosmology and Astroparticle Physics, 6, 036

Sen, S., \& Sen, A. A. 2003, ApJ, 588, 1

Senovilla, J. M. M., Sopuerta, C. F., \& Szekeres, P. 1998, General Relativity and Gravitation, 30, 389

Seo, H.-J., \& Eisenstein, D. J. 2003, ApJ, 598, 720

Shafieloo, A., Kim, A. G., \& Linder, E. V. 2012, Phys. Rev. D, 85,123530

Sharov, G. S. 2016, Journal of Cosmology and Astroparticle Physics, 6, 023

Shi, D., \& Baugh, C. M. 2016, MNRAS, 459, 3540

Shi, K., Huang, Y. F., \& Lu, T. 2012, MNRAS, 426, 2452

Silvestri, A., \& Trodden, M. 2009, Reports on Progress in Physics, 72, 096901

Slepian, Z., Gott, J. R., \& Zinn, J. 2014, MNRAS, 438, 1948

Sola, J., de Cruz Perez, J., \& Gomez-Valent, A. 2017a, ArXiv e-prints, arXiv:1703.08218

Sola, J., Gomez-Valent, A., \& de Cruz Perez, J. 2017b, ApJ, 836, 43

-. 2017c, ArXiv e-prints, arXiv:1705.06723

Song, Y.-S., Hu, W., \& Sawicki, I. 2007, Phys. Rev. D, 75, 044004

Sotiriou, T. P., \& Faraoni, V. 2010, Rev. Mod. Phys., 82, 451

Starobinsky, A. A. 2007, Soviet Journal of Experimental and Theoretical Physics Letters, 86, 157

Sunyaev, R. A., \& Zeldovich, Y. B. 1970, Astrophysics and Space Science, 7,3

Susskind, L. 1995, Journal of Mathematical Physics, 36, 6377

't Hooft, G. 1993, ArXiv General Relativity and Quantum Cosmology e-prints, gr-qc/9310026

't Hooft, G. 2001, in Basics and Highlights in Fundamental Physics, ed. A. Zichichi, 72-100

Tocchini-Valentini, D., \& Amendola, L. 2002, Phys. Rev. D, 65, 063508

Tsujikawa, S. 2007, Phys. Rev. D, 76, 023514

Turner, M. S., \& White, M. 1997, Phys. Rev. D, 56, R4439

Unzicker, A., \& Case, T. 2005, ArXiv Physics e-prints, physics $/ 0503046$

Urban, F. R., \& Zhitnitsky, A. R. 2009a, Phys. Rev. D, 80, 063001

—. 2009b, Journal of Cosmology and Astroparticle Physics, 9, 018

—. 2010a, Physics Letters B, 688, 9

—. 2010b, Nuclear Physics B, 835, 135
Veneziano, G. 1979, Nuclear Physics B, 159, 213

Wang, Y., Freese, K., Gondolo, P., \& Lewis, M. 2003, ApJ, 594 , 25

Wang, Y., Wands, D., Xu, L., De-Santiago, J., \& Hojjati, A. 2013, Phys. Rev. D, 87, 083503

Wasserstein, R. L., \& Lazar, N. A. 2016, The American Statistician, 70, 129

Watson, C. R., \& Scherrer, R. J. 2003, Phys. Rev. D, 68, 123524

Wei, H., \& Cai, R.-G. 2008, Physics Letters B, 660, 113

Weinberg, D. H., Mortonson, M. J., Eisenstein, D. J., et al. 2013, Phys. Rep., 530, 87

Weinberg, S. 1989, Rev. Mod. Phys., 61, 1

Weller, J., \& Albrecht, A. 2002, Phys. Rev. D, 65, 103512

Wetterich, C. 1988, Nuclear Physics B, 302, 668

Witten, E. 1979, Nuclear Physics B, 156, 269

Wu, H.-Y., \& Huterer, D. 2017, MNRAS, 471, 4946

Wu, P., \& Yu, H. 2011, European Physical Journal C, 71, 1552

$\mathrm{Xu}$, L. 2012, European Physical Journal C, 72, 2134

—. 2013, Phys. Rev. D, 87, 043525

-. 2014, Journal of Cosmology and Astroparticle Physics, 2, 048

Xu, Y.-Y., \& Zhang, X. 2016, ArXiv e-prints, arXiv:1607.06262

Zhang, B. R., Childress, M. J., Davis, T. M., et al. 2017, MNRAS, 471, 2254

Zhang, J.-F., Zhao, M.-M., Cui, J.-L., \& Zhang, X. 2014, European Physical Journal C, 74, 3178

Zhang, J.-F., Zhao, M.-M., Li, Y.-H., \& Zhang, X. 2015, Journal of Cosmology and Astroparticle Physics, 4, 038

Zhang, X., \& Wu, F.-Q. 2007, Phys. Rev. D, 76, 023502

Zhang, Y., Li, H., Wu, X., Wei, H., \& Cai, R.-G. 2007, ArXiv e-prints, arXiv:0708.1214

Zhao, G.-B., Li, B., \& Koyama, K. 2011, Phys. Rev. D, 83, 044007

Zhao, G.-B., Wang, Y., Ross, A. J., et al. 2016, MNRAS, 457, 2377

Zhao, G.-B., Raveri, M., Pogosian, L., et al. 2017, Nature

Astronomy, 1, 627

Zhu, Z.-H., \& Fujimoto, M.-K. 2002, ApJ, 581, 1

-. 2003, ApJ, 585, 52

Zhu, Z.-H., Fujimoto, M.-K., \& He, X.-T. 2004, ApJ, 603, 365

Zimdahl, W., Pavn, D., \& Chimento, L. P. 2001, Physics Letters $\mathrm{B}, 521,133$ 